\title{
Studies Toward the Total Synthesis of Wiedemannic Acid
}

\author{
Louis Barriault* and Effie L. O. Sauer \\ Department of Chemistry, University of Ottawa, Ottawa, K1N 6N5
}

Experimental Procedures and Copies of ${ }^{1} \mathrm{H}$ and ${ }^{13} \mathrm{C}$ NMR for $\mathbf{1}$ and 13-31

General. All reactions were performed under nitrogen or argon atmosphere with flame-dried glassware. All solvents were distilled prior to use; diethyl ether and THF over sodium and benzophenone. Toluene and dichloromethane (DCM) were dried over calcium hydride. Column chromatography was performed with 60 silica gel (230-400 mesh, Merck). ${ }^{1} \mathrm{H}$ and ${ }^{13} \mathrm{C}$ NMR were recorded on Bruker instruments at 500 and $125 \mathrm{MHz}$, respectively, except where noted. IR spectra were recorded with a Bomen instrument. Melting points are uncorrected. The high-resolution mass spectra were recorded at The University of Ottawa Mass Spectrum Center. 


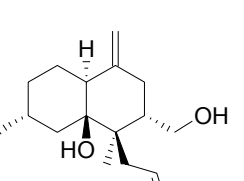

4-Allyl-3-hydroxymethyl-4,6-dimethyl-1-methylene-octahydronaphthalen-4a-ol (13): To a solution of $20 \mathrm{mg}(0.053 \mathrm{mmol}) \mathbf{1 0}$ in 0.4 $\mathrm{mL}$ THF at room temperature was added TBAF $(0.1 \mathrm{~mL}$ of a $1.0 \mathrm{M}$ solution in THF, $1.000 \mathrm{mmol})$. After stirring for 2 hours the reaction was concentrated and purified by silica gel flash chromatography $(40 \%$ EtOAc/hexanes) to give $13 \mathrm{mg}$ of white crystals $(91 \%) .{ }^{1} \mathrm{H} \mathrm{NMR}\left(\mathrm{CDCl}_{3}, 500 \mathrm{MHz}\right)$ : 6.19-6.10 (m, 1H), 5.01 (dddd, $J=17.2,0.7,0.7,0.7$ Hz, 1H), 4.97 (dddd, $J=10.2,1.1$, $1.1,1.1 \mathrm{~Hz}, 1 \mathrm{H}), 4.91(\mathrm{~d}, \mathrm{~J}=1.5 \mathrm{~Hz}, 1 \mathrm{H}), 4.65(\mathrm{~s}, 1 \mathrm{H}), 3.92-3.89(\mathrm{~m}, 1 \mathrm{H}), 3.39-3.35(\mathrm{~m}$, $1 \mathrm{H}), 2.57-2.50(\mathrm{~m}, 1 \mathrm{H}), 2.45\left(\mathrm{dd}, \mathrm{J}_{\mathrm{AB}}=15.6 \mathrm{~Hz}, \mathrm{~J}_{\mathrm{AX}}=7.6 \mathrm{~Hz}, 1 \mathrm{H}\right), 2.20\left(\mathrm{dd}, \mathrm{J}_{\mathrm{AB}}=15.6\right.$ $\left.\mathrm{Hz}, \mathrm{J}_{\mathrm{AX}}=7.3 \mathrm{~Hz}, 1 \mathrm{H}\right), 2.18-2.15(\mathrm{~m}, 1 \mathrm{H}), 2.07-2.01(\mathrm{~m}, 2 \mathrm{H}), 1.81(\mathrm{ddd}, \mathrm{J}=13.2,3.3,2.3$ $\mathrm{Hz}, 1 \mathrm{H}), 1.77-1.69(\mathrm{~m}, 2 \mathrm{H}), 1.60-1.52(\mathrm{~m}, 2 \mathrm{H}), 1.50(\mathrm{~d}, \mathrm{~J}=1.1 \mathrm{~Hz}, 1 \mathrm{H}), 1.15$ (dd, J = 4.8, 4.8 Hz, 1H), 1.09 (dd, J = 13.0, $13.0 \mathrm{~Hz}, 1 \mathrm{H}), 0.91(\mathrm{~s}, 3 \mathrm{H}), 0.88(\mathrm{~d}, \mathrm{~J}=6.5 \mathrm{~Hz}, 3 \mathrm{H})$, 0.86-0.83 (m, 1H); ${ }^{13} \mathrm{C} \mathrm{NMR}\left(\mathrm{CDCl}_{3}, 500 \mathrm{MHz}\right): 148.97,138.34,115.77,108.85,78.57$, $64.21,44.12,43.72,43.52,40.06,39.66,35.87,34.03,27.53,24.61,22.52,18.33$; IR (neat): 3363 (br, m), $3076(\mathrm{w}), 2950$ (m), 2357 (m), 2339 (m), 1632 (w), 1356 (s), 1050 (w), 1002 (w), 888 (w); HRMS (EI) m/z: calculated 264.2089 for [M] $]^{+}$, found 264.2099 ; $\mathrm{mp}=75.8-77.6^{\circ} \mathrm{C}$.

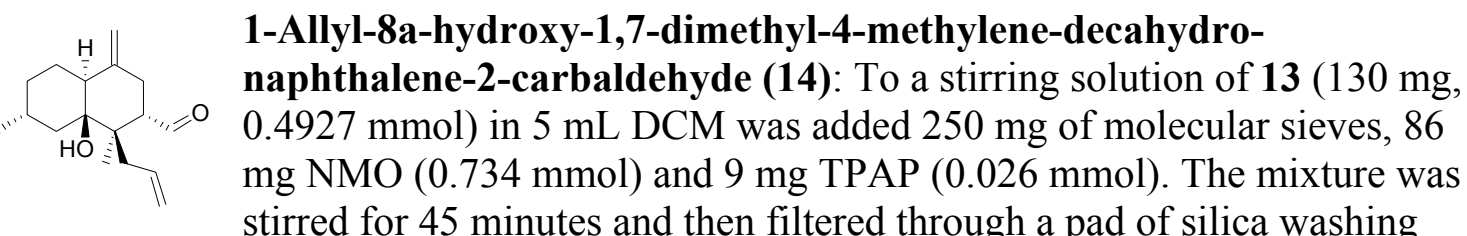
first with DCM, then a 10:1 EtOAc/MeOH solution and then again with DCM. The filtrate was concentrated and purified by silica gel flash chromatography $(5 \% \rightarrow 10 \%$ EtOAc/hexanes) to give $122 \mathrm{mg}$ of a white solid (95\%). ${ }^{1} \mathrm{H} \mathrm{NMR}\left(\mathrm{CDCl}_{3}, 300 \mathrm{MHz}\right)$ : $9.85(\mathrm{~d}, \mathrm{~J}=2.1 \mathrm{~Hz}, 1 \mathrm{H}), 6.16-6.02(\mathrm{~m}, 1 \mathrm{H}), 5.03(\mathrm{~d}, \mathrm{~J}=17.1 \mathrm{~Hz}, 1 \mathrm{H}), 5.02(\mathrm{~d}, \mathrm{~J}=10.1$ $\mathrm{Hz}, 1 \mathrm{H}), 4.89$ (d, J = 1.5 Hz, 1H), 4.67 (d, J = 1.4 Hz, 1H), 2.93 (ddd, J = 12.9, 4.1, 2.1 $\mathrm{Hz}, 1 \mathrm{H}), 2.56(\mathrm{dd}, \mathrm{J}=15.2,7.9 \mathrm{~Hz}, 1 \mathrm{H}), 2.42-2.14(\mathrm{~m}, 4 \mathrm{H}), 1.82-1.42(\mathrm{~m}, 6 \mathrm{H}), 1.10-1.02$ $(\mathrm{m}, 1 \mathrm{H}), 1.06(\mathrm{~s}, 3 \mathrm{H}), 0.92-0.77(\mathrm{~m}, 1 \mathrm{H}), 0.87(\mathrm{~d}, \mathrm{~J}=6.3 \mathrm{~Hz}, 3 \mathrm{H}) ;{ }^{13} \mathrm{C} \mathrm{NMR}\left(\mathrm{CDCl}_{3}, 300\right.$ MHz): 205.15, 147.13, 136.99, 117.55, 110.09, 78.27, 53.32, 44.84, 43.41, 40.46, 38.99, 33.73, 32.19, 27.47, 24.42, 22.40, 18.41; IR (neat): 3556 (m), 3075 (w), 2948 (m), 2926 (s), 2867 (m), $2846(\mathrm{~m}), 2739(\mathrm{w}), 1715$ (s), $1644(\mathrm{~m}), 1445(\mathrm{~m}), 1347(\mathrm{~m}), 998(\mathrm{~m}), 945$ (m), 913 (s); HRMS (EI) m/z: calculated 262.1933 for [M] $]^{+}$, found 262.1937; mp = 52.8$54.3{ }^{\circ} \mathrm{C}$.

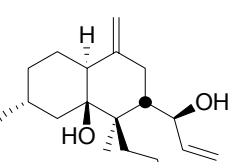

\section{4-Allyl-3-(1-hydroxy-allyl)-4,6-dimethyl-1-methylene-octahydro-} naphthalen-4a-ol (15): Method A: A solution of 14 (122 mg, 0.465 $\mathrm{mmol}$ ) in $4 \mathrm{~mL}$ THF was cooled to $-78^{\circ} \mathrm{C}$. Vinylmagnesium bromide (1.2 $\mathrm{mL}$ of a $0.9 \mathrm{M}$ solution, $1.080 \mathrm{mmol}$ ) was added dropwise and the reaction stirred for 45 minutes. A saturated solution of aqueous $\mathrm{NH}_{4} \mathrm{Cl}$ was added and the reaction warmed to room temperature. The aqueous phase was extracted three times with EtOAc and the combined organic layers dried with $\mathrm{MgSO}_{4}$, filtered and concentrated. Purification by silica gel flash chromatography $(2 \% \rightarrow 4 \%$ EtOAc/hexanes) yielded $90 \mathrm{mg}$ of a colourless oil as an inseparable mixture of $\mathbf{1 5}$ and 
acetal 16. Method B (for the purpose of obtaining 15 in pure form): To a solution of 20 (7 $\mathrm{mg}, 0.019 \mathrm{mmol})$ in $1.0 \mathrm{~mL}$ THF was added TBAF $(0.04 \mathrm{~mL}$ of a $1.0 \mathrm{M}$ solution in THF, $0.040 \mathrm{mmol})$. After stirring for 24 hours the reaction was concentrated and the crude oil purified by silica gel flash chromatography (10\% EtOAc/hexanes) to yield $5 \mathrm{mg}$ of a colourless oil (89\%). ${ }^{1} \mathrm{H}$ NMR $\left(\mathrm{CDCl}_{3}, 500 \mathrm{MHz}\right): 6.14-6.05(\mathrm{~m}, 1 \mathrm{H}), 5.90$ (ddd, J = 17.2, 10.6, $4.5 \mathrm{~Hz}, 1 \mathrm{H}), 5.20$ (ddd, J = 17.1, 1.6, $1.4 \mathrm{~Hz}, 1 \mathrm{H}), 5.12$ (ddd, J = 10.5, 1.6, $1.3 \mathrm{~Hz}$, $1 \mathrm{H}), 5.00$ (dddd, J = 17.2, 1.6, 1.5, $1.5 \mathrm{~Hz}, 1 \mathrm{H}), 4.98-4.94(\mathrm{~m}, 1 \mathrm{H}), 4.84(\mathrm{~d}, \mathrm{~J}=1.6 \mathrm{~Hz}$, $1 \mathrm{H}), 4.62(\mathrm{~d}, \mathrm{~J}=1.4 \mathrm{~Hz}, 1 \mathrm{H}), 4.55(\mathrm{~s}$, br. $1 \mathrm{H}), 2.52(\mathrm{dd}, \mathrm{J}=15.7,7.6 \mathrm{~Hz}, 1 \mathrm{H}), 2.33(\mathrm{dd}, \mathrm{J}$ $=15.7,7.2 \mathrm{~Hz}, 1 \mathrm{H}), 2.27(\mathrm{dd}, \mathrm{J}=14.0,13.2 \mathrm{~Hz}, 1 \mathrm{H}), 2.20-2.17(\mathrm{~m}, 1 \mathrm{H}), 2.12(\mathrm{dd}, \mathrm{J}=$ 13.3, 3.6 Hz, 1H), $2.04(\mathrm{dd}, \mathrm{J}=14.0,2.8 \mathrm{~Hz}, 1 \mathrm{H}), 1.82-1.78(\mathrm{~m}, 1 \mathrm{H}), 1.73-1.68(\mathrm{~m}, 2 \mathrm{H})$, 1.59-1.48 (m, 4H), 1.17-1.14 (m, 1H), 1.15 (s, 3H), 0.88 (d, J = 6.4 Hz, 3H), 0.88-0.81 $(\mathrm{m}, 1 \mathrm{H}) ;{ }^{13} \mathrm{C} \mathrm{NMR}\left(\mathrm{CDCl}_{3}, 500 \mathrm{MHz}\right): 149.67,141.62,138.13,115.88,113.22,108.29$, 78.93, 70.47, 44.98, 43.95, 43.90, 40.23, 39.75, 33.85, 30.03, 27.45, 24.51, 22.42, 19.49; IR (neat): 3427 (m, br), 3075 (w), 2948 (s), 2925 (s), 2867 (m), 1727 (w), 1637 (w), 1455 (m), 1380 (w), 1204 (w), 1111 (m), 1087 (m), 993 (m), 917 (s); HRMS (EI) m/z: calculated 272.2140 for $\left[\mathrm{M}-\mathrm{H}_{2} \mathrm{O}\right]^{+}$, found 272.2134 .

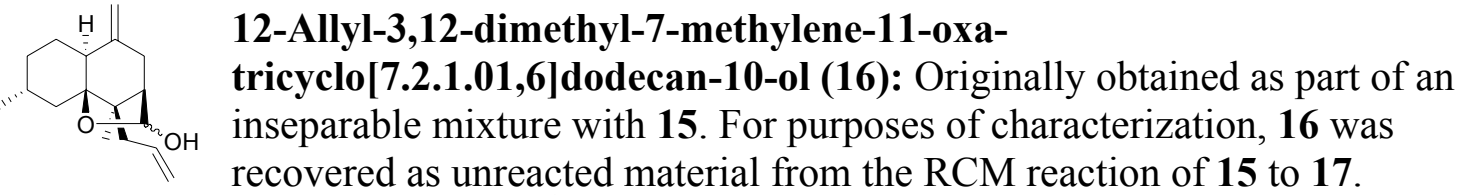
Mixture of isomers ( 2:1 ratio): ${ }^{1} \mathrm{H} \mathrm{NMR}\left(\mathrm{CDCl}_{3}, 300 \mathrm{MHz}\right): 5.94-5.69(\mathrm{~m}, 1 \mathrm{H}), 5.37$ and 5.11-5.05 (s, br and $\mathrm{m}, 1 \mathrm{H}), 5.11-5.05(\mathrm{~m}, 2 \mathrm{H}), 4.93(\mathrm{~s}, 1 \mathrm{H}), 4.82(\mathrm{~s}, 1 \mathrm{H}), 2.87-2.78$ $(\mathrm{m}, 1 \mathrm{H}), 2.58-2.45(\mathrm{~m}, 2 \mathrm{H}), 2.34-1.52(\mathrm{~m}, 9 \mathrm{H}), 1.49-1.32(\mathrm{~m}, 1 \mathrm{H}), 1.07$ and $1.01(\mathrm{~s}$ and $\mathrm{s}$, $3 \mathrm{H}), 0.94-0.77(\mathrm{~m}, 1 \mathrm{H}), 0.88$ (d, J = 6.3 Hz, 3H); ${ }^{13} \mathrm{C} \mathrm{NMR}\left(\mathrm{CDCl}_{3}, 300 \mathrm{MHz}\right): 149.37$, 147.01, 136.46, 134.75, 118.74, 118.37, 112.57, 112.06, 101.19, 100.04, 89.71, 86.17, 47.94, 45.98, 45.54, 43.42, 43.01, 42.62, 39.90, 39.54, 38.28, 37.84, 35.32, 34.23, 34.21, 31.57, 28.95, 28.81, 26.90 (2C), 23.10, 23.04, 17.64, 17.09; IR (neat): 3400 (s, br), 3075 (w), 2993 (w), 2948 (s), 2925 (s), 2867 (s), 2843 (m), 1636 (m), 1447 (m), 1381 (m), $1204(\mathrm{w}), 1157$ (w), 1139 (w), 1112 (m), 1088 (m), 922 (s); HRMS (EI) m/z: calculated 262.1933 for $[\mathrm{M}]^{+}$, found 262.1954 .

4a,6-Dimethyl-9-methylene-4,4a,5,6,7,8,8a,9,10,10a-decahydro-1H-
phenanthrene-1,4b-diol (17): A solution of 15 (17 mg, $0.0585 \mathrm{mmol})$, as
an inseparable mixture with acetal 16, in $7 \mathrm{~mL}$ DCM was degassed under
argon for 20 minutes. Grubb's first generation catalyst (4 mg, $0.05 \mathrm{mmol})$ was added and the solution went from pink to dark brown over time. After 45 minutes no further reaction was observed and the solution was concentrated and purified by silica gel flash chromatography $(10 \% \rightarrow 20 \%$ EtOAc/hexanes $)$ to give $10 \mathrm{mg}(65 \%)$ of a 17 as a white solid (as well as $9 \mathrm{mg}$ of unreacted acetal 16). ${ }^{1} \mathrm{H} \mathrm{NMR}\left(\mathrm{CDCl}_{3}, 300 \mathrm{MHz}\right): 5.74-$ $5.70(\mathrm{~m}, 1 \mathrm{H}), 5.59(\mathrm{~d}, \mathrm{~J}=10.1 \mathrm{~Hz}, 1 \mathrm{H}), 4.97(\mathrm{~d}, \mathrm{~J}=1.3 \mathrm{~Hz}, 1 \mathrm{H}), 4.70(\mathrm{~s}, 1 \mathrm{H}), 3.82(\mathrm{~d}, \mathrm{~J}=$ $7.7 \mathrm{~Hz}, 1 \mathrm{H}), 2.66(\mathrm{dd}, \mathrm{J}=13.3,4.1 \mathrm{~Hz}, 1 \mathrm{H}), 2.48(\mathrm{~d}, \mathrm{~J}=18.9 \mathrm{~Hz}, 1 \mathrm{H}), 2.28(\mathrm{~d}, \mathrm{~J}=10.0$ $\mathrm{Hz}, 1 \mathrm{H}), 1.99$ (dd, J = 14.8, 13.1 Hz, 1H), 1.73-1.68 (m, 3H), 1.65-1.59 (m, 3H), 1.561.49 (m, 3H), 1.39 (s, 1H), 1.08 (dd, J = 12.9, $12.5 \mathrm{~Hz}, 1 \mathrm{H}), 0.99$ (s, 3H), 0.87 (d, J = 6.3 $\mathrm{Hz}, 3 \mathrm{H}) ;{ }^{13} \mathrm{C} \mathrm{NMR}\left(\mathrm{CDCl}_{3}, 500 \mathrm{MHz}\right): 148.69,128.69,128.17,110.06,76.43,71.47$, 
45.75, 43.15, 41.91, 38.15, 35.00, 34.01, 32.46, 27.39, 24.71, 22.42, 15.06; IR (neat): 3349 (s), 2944 (s), 2921 (s), 2867 (m), 1646 (w), 1536 (w), 1518 (m), 1443 (m), 1393 (m), 1143 (w), 1097 (w), 1041 (s), 984 (s), 942 (m), 888 (m); HRMS (EI) m/z: calculated 244.1827 for $\left[\mathrm{M}-\mathrm{H}_{2} \mathrm{O}\right]^{+}$, found $244.1834 ; \mathrm{mp}=138.2-140.1^{\circ} \mathrm{C}$.

\section{H 1-Allyl-1,7-dimethyl-4-methylene-8a-trimethylsilanyloxy-decahydro- naphthalene-2-carbaldehyde (19): A solution of $\mathbf{1 3}$ (147 mg, 0.559 $\mathrm{mmol})$ in $5 \mathrm{~mL}$ THF was cooled to $-78^{\circ} \mathrm{C}$. Freshly distilled TMS-Cl $(0.28$ $\mathrm{mL}, 2.206 \mathrm{mmol}$ ) was then added dropwise followed by KHMDS (407} $\mathrm{mg}, 2.040 \mathrm{mmol}$ ) in one portion. After stirring for 1.5 hours the reaction was quenched with $\mathrm{NaHCO}_{3}$. The reaction mixture was extracted three times with EtOAc and the combined organic layers dried with $\mathrm{MgSO}_{4}$, filtered and concentrated. The crude bissilated material was dissolved in $5 \mathrm{~mL}$ of $\mathrm{MeOH}$ and cooled to $0{ }^{\circ} \mathrm{C}$. Anhydrous potassium carbonate was added $(75 \mathrm{mg}, 0.543 \mathrm{mmol})$ and the reaction stirred for 30 minutes. The reaction was then diluted with water, extracted three times with DCM and the combined organic layers dried with $\mathrm{MgSO}_{4}$, filtered and concentrated. The crude oil was then dissolved in $5 \mathrm{~mL}$ of DCM and put under an atmosphere of nitrogen. Molecular sieves $(4 \AA)$ were added $(250 \mathrm{mg})$ followed by NMO ( $87 \mathrm{mg}, 0.747 \mathrm{mmol})$ and TPAP (5 $\mathrm{mg}, 0.014 \mathrm{mmol})$. After stirring for 20 minutes, the reaction was filtered over a pad of silica and washed with a 5\% solution of $\mathrm{MeOH}$ in EtOAc followed by DCM. The filtrate was concentrated and purification by silica gel flash chromatography $(5 \%$

EtOAc/hexanes) yielded $165 \mathrm{mg}$ of the aldehyde 19 as a white solid (88\% over three steps). ${ }^{1} \mathrm{H} \mathrm{NMR}\left(\mathrm{CDCl}_{3}, 500 \mathrm{MHz}\right): 9.83(\mathrm{~d}, \mathrm{~J}=3.4 \mathrm{~Hz}, 1 \mathrm{H}), 5.82-5.74(\mathrm{~m}, 1 \mathrm{H}), 5.08$ (d, $\mathrm{J}=1.0 \mathrm{~Hz}, 1 \mathrm{H}), 5.06-5.05(\mathrm{~m}, 1 \mathrm{H}), 4.74(\mathrm{~d}, \mathrm{~J}=1.6 \mathrm{~Hz}, 1 \mathrm{H}), 4.53(\mathrm{~d}, \mathrm{~J}=1.6 \mathrm{~Hz}, 1 \mathrm{H})$, $2.77(\mathrm{ddd}, \mathrm{J}=13.1,3.7,3.7 \mathrm{~Hz}, 1 \mathrm{H}), 2.43\left(\mathrm{dd}, \mathrm{J}_{\mathrm{AB}}=14.4,6.7 \mathrm{~Hz}, 1 \mathrm{H}\right), 2.34(\mathrm{dd}, \mathrm{J}=13.8$, $13.8 \mathrm{~Hz}, 1 \mathrm{H}), 2.28\left(\mathrm{dd}, \mathrm{J}_{\mathrm{AB}}=14.4,8.3 \mathrm{~Hz}, 1 \mathrm{H}\right), 2.11-2.06(\mathrm{~m}, 2 \mathrm{H}), 1.77-1.71(\mathrm{~m}, 2 \mathrm{H})$, $1.67-1.58(\mathrm{~m}, 2 \mathrm{H}), 1.54-1.46(\mathrm{~m}, 1 \mathrm{H}), 1.15(\mathrm{~s}, 3 \mathrm{H}), 1.07(\mathrm{dd}, \mathrm{J}=13.4,12.4 \mathrm{~Hz}, 1 \mathrm{H})$, 0.92-0.83 (m, 1H), $0.88(\mathrm{~d}, \mathrm{~J}=6.6 \mathrm{~Hz}, 3 \mathrm{H}), 0.13(\mathrm{~s}, 9 \mathrm{H}) ;{ }^{13} \mathrm{C} \mathrm{NMR}\left(\mathrm{CDCl}_{3}, 500 \mathrm{MHz}\right)$ : 205.68, 147.13, 136.18, 118.74, 107.89, 84.26, 56.21, 45.43, 43.96, 40.33, 38.90, 33.88, 33.01, 28.21, 24.69, 22.33, 16.48, 2.90 (3C); IR (neat): 2950 (s), 2925 (s), 1715 (s), 1443 (m), 1249 (s), 1176 (w), 1127 (m), 1090 (m), 894 (s); HRMS (EI) m/z: calculated 334.2328 for $[\mathrm{M}]^{+}$, found 334.2327 ; $\mathrm{mp}=59.1-60.6{ }^{\circ} \mathrm{C}$.

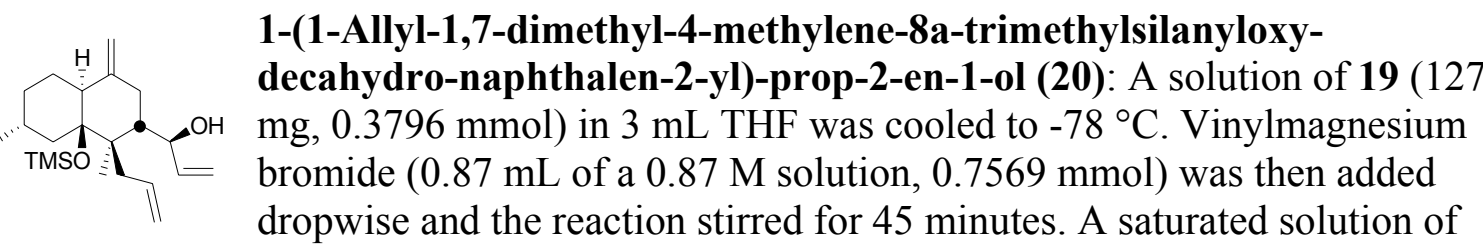

aqueous $\mathrm{NH}_{4} \mathrm{Cl}$ was added and the reaction warmed to room temperature. The aqueous phase was extracted three times with EtOAc and the combined organic layers dried with $\mathrm{MgSO}_{4}$, filtered and concentrated. No purification was required and $133 \mathrm{mg}$ of a white solid $(97 \%)$ was obtained. ${ }^{1} \mathrm{H}$ NMR $\left(\mathrm{CDCl}_{3}, 500 \mathrm{MHz}\right): 6.04-5.99(\mathrm{~m}, 1 \mathrm{H}), 5.88-5.83(\mathrm{~m}$, $1 \mathrm{H}), 5.17(\mathrm{~d}, \mathrm{~J}=17.2 \mathrm{~Hz}, 1 \mathrm{H}), 5.10(\mathrm{~d}, \mathrm{~J}=10.6 \mathrm{~Hz}, 1 \mathrm{H}), 5.03(\mathrm{~d}, \mathrm{~J}=20.8 \mathrm{~Hz}, 1 \mathrm{H}), 4.99$ $(\mathrm{d}, \mathrm{J}=11.2 \mathrm{~Hz}, 1 \mathrm{H}), 4.66(\mathrm{~s}, 2 \mathrm{H}), 4.45(\mathrm{~s}, 1 \mathrm{H}), 2.49$ (dd, J = 15.1, $5.5 \mathrm{~Hz}, 1 \mathrm{H}), 2.21$ (dd, $\mathrm{J}=12.7,12.7 \mathrm{~Hz}, 1 \mathrm{H}), 2.11-2.02(\mathrm{~m}, 3 \mathrm{H}), 1.91(\mathrm{~d}, \mathrm{~J}=13.4 \mathrm{~Hz}, 1 \mathrm{H}), 1.75-1.69(\mathrm{~m}, 2 \mathrm{H})$, 
1.59-1.48 (m, 3H), 1.24-1.06 (m, 2H), $1.14(\mathrm{~s}, 3 \mathrm{H}), 0.87-0.85(\mathrm{~m}, 1 \mathrm{H}), 0.87(\mathrm{~d}, \mathrm{~J}=3.5$ $\mathrm{Hz}, 3 \mathrm{H}), 0.08(\mathrm{~s}, 9 \mathrm{H}) ;{ }^{13} \mathrm{C} \mathrm{NMR}\left(\mathrm{CDCl}_{3}, 500 \mathrm{MHz}\right): 149.85,142.38,138.27,115.70$, $112.91,106.11,85.33,71.42,47.75,46.17,45.03,39.99$, 39.66, 34.08, 30.40, 28.21, 24.84, 22.41, 17.33, 2.96 (3C); IR (neat): 3435 (br, w), 3081 (w), 2950 (s), 2926 (s), 2868 (m), 1646 (w), 1455 (w), 1248 (s), 1119 (s), 1092 (m), 1063 (m), 913 (m), 883 (m), 836 (s), $750(\mathrm{w})$; HRMS (EI) m/z: calculated 362.2641 for $[\mathrm{M}]^{+}$, found 362.2643; $\mathrm{mp}=61.4-$ $63.8^{\circ} \mathrm{C}$.

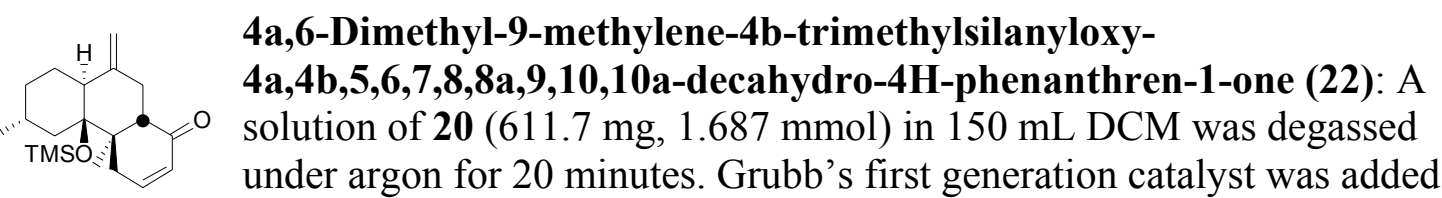

(52 mg, $0.063 \mathrm{mmol}$ ) and the resulting pink solution was stirred for 30 minutes and then concentrated. The crude allylic alcohol 21 was redissolved in $15 \mathrm{~mL}$ DCM and $840 \mathrm{mg}$ of $4 \AA$ molecular sieves, $400 \mathrm{mg}$ NMO $(3.414 \mathrm{mmol})$ and $15 \mathrm{mg}$ TPAP $(0.043 \mathrm{mmol})$ were added in succession. The resulting dark brown solution was stirred for 45 minutes and then filtered over a pad of silica, washed twice with a $5 \%$ solution of $\mathrm{MeOH}$ in EtOAc followed by several DCM washes. The filtrate was concentrated and purified by silica gel flash chromatography (100\% hexanes $\rightarrow 8 \%$ EtOAc/hexanes) to yield $511 \mathrm{mg}$ of enone 22 as a colourless oil (91\% over 2 steps). ${ }^{1} \mathrm{H}$ NMR $\left(\mathrm{CDCl}_{3}, 300 \mathrm{MHz}\right): 6.82-6.76$ $(\mathrm{m}, 1 \mathrm{H}), 5.93(\mathrm{dd}, \mathrm{J}=10.0,2.9 \mathrm{~Hz}, 1 \mathrm{H}), 4.76(\mathrm{~s}, 1 \mathrm{H}), 4.49(\mathrm{~s}, 1 \mathrm{H}), 2.80(\mathrm{dd}, \mathrm{J}=12.3,4.0$ $\mathrm{Hz}, 1 \mathrm{H}), 2.64-2.53(\mathrm{~m}, 2 \mathrm{H}), 2.17-2.03(\mathrm{~m}, 3 \mathrm{H}), 1.78-1.58(\mathrm{~m}, 4 \mathrm{H}), 1.47$ (dddd, J = 12.5, 3.4, 3.4, 3.4 Hz, 1H), 1.09 (dd, J = 13.6 Hz, 1H), $1.03(\mathrm{~s}, 3 \mathrm{H}), 0.93-0.80(\mathrm{~m}, 1 \mathrm{H}), 0.86(\mathrm{~d}$, $\mathrm{J}=6.4 \mathrm{~Hz}, 3 \mathrm{H}), 0.09(\mathrm{~s}, 9 \mathrm{H}) ;{ }^{13} \mathrm{C} \mathrm{NMR}\left(\mathrm{CDCl}_{3}, 300 \mathrm{MHz}\right): 202.17,147.72,146.65$, $128.11,107.75,83.23,50.26,46.59,43.30,38.20,34.30,33.87,30.95,27.93,24.51$, 22.19, 16.34, 2.87 (3C); IR (neat): 2950 (s), 2931 (s), 2866 (m), 1729 (w), 1681 (s), 1647 (w), $1456(\mathrm{w}), 1387(\mathrm{~m}), 1250(\mathrm{~m}), 1194(\mathrm{~m}), 1135(\mathrm{~m}), 1058(\mathrm{~m}), 836(\mathrm{~s}), 751(\mathrm{w})$; HRMS (EI) $\mathrm{m} / \mathrm{z}$ : calculated 332.2172 for $[\mathrm{M}]^{+}$, found 332.2158 .

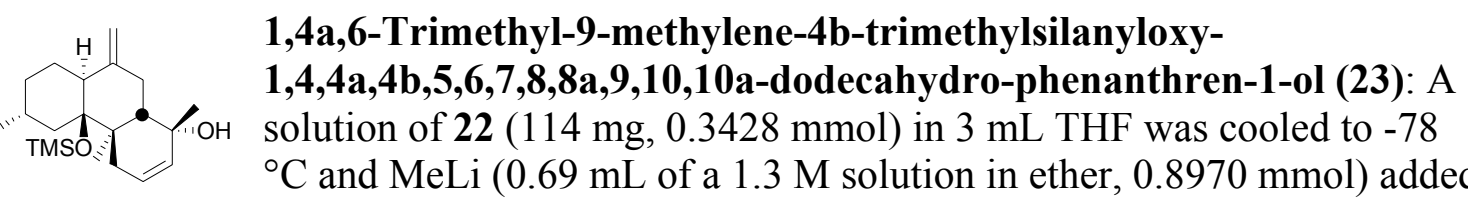

dropwise. After 20 minutes the reaction was quenched with water and warmed to room temperature. The aqueous phase was extracted three times with EtOAc and the combined organic layers dried with $\mathrm{MgSO}_{4}$, filtered and concentrated. Purification by silica gel flash chromatography (10\% EtOAc/hexanes) gave $117 \mathrm{mg}$ of a white solid $(98 \%) .{ }^{1} \mathrm{H}$ NMR $\left(\mathrm{CDCl}_{3}, 500 \mathrm{MHz}\right)$ : 5.70-5.67 (m, 1H), 5.60-5.57 (m, 1H), $4.73(\mathrm{~s}, 1 \mathrm{H}), 4.48(\mathrm{~s}$, $1 \mathrm{H}), 2.39-2.28(\mathrm{~m}, 2 \mathrm{H}), 2.12-2.08(\mathrm{~m}, 2 \mathrm{H}), 1.96(\mathrm{dd}, \mathrm{J}=12.8,4.0 \mathrm{~Hz}, 1 \mathrm{H}), 1.80(\mathrm{dd}, \mathrm{J}=$ 17.3, $5.9 \mathrm{~Hz}, 1 \mathrm{H}), 1.72-1.68(\mathrm{~m}, 1 \mathrm{H}), 1.63-1.44(\mathrm{~m}, 4 \mathrm{H}), 1.21(\mathrm{~s}, 3 \mathrm{H}), 1.18(\mathrm{dd}, \mathrm{J}=9.3$, $9.3 \mathrm{~Hz}, 1 \mathrm{H}), 1.14-1.11(\mathrm{~m}, 1 \mathrm{H}), 1.13(\mathrm{~s}, 3 \mathrm{H}), 0.91-0.83(\mathrm{~m}, 1 \mathrm{H}), 0.86(\mathrm{~d}, \mathrm{~J}=6.4 \mathrm{~Hz}, 3 \mathrm{H})$, 0.05 (s, 9H); ${ }^{13} \mathrm{C} \mathrm{NMR}\left(\mathrm{CDCl}_{3}, 500 \mathrm{MHz}\right): 150.01,132.92,126.03,106.36,83.80,69.53$, 44.72, 43.67, 40.80, 38.43, 34.07, 32.82, 31.95, 29.82, 28.07, 24.90, 22.29, 16.81, 2.77 (3C); IR (neat): 3450 (br, m), 3083 (w), 3019 (m), 2949 (s), 2871 (m), 2847 (m), 1646 (m), 1455 (m), 1368 (m), 1249 (s), 1183 (m), 1149 (m), 1061 (s), 1020 (m), 913 (m), 838 
(s), $751(\mathrm{~m})$; HRMS (EI) m/z: calculated 348.2485 for $[\mathrm{M}]^{+}$, found $348.2465 ; \mathrm{mp}=92.1$ $93.2{ }^{\circ} \mathrm{C}$.

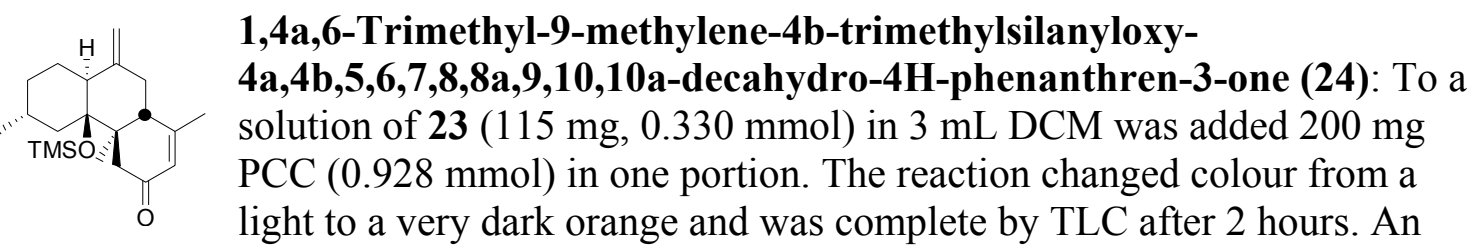

equal volume of ether was then added and the resulting precipitate filtered off over celite, rinsing thoroughly with additional ether. The filtrate was concentrated and purified by silica gel flash chromatography (10\% EtOAc/hexanes). The resulting white solid had a mass of $104 \mathrm{mg}(91 \%) .{ }^{1} \mathrm{H}$ NMR $\left(\mathrm{CDCl}_{3}, 500 \mathrm{MHz}\right): 5.84(\mathrm{~s}, 1 \mathrm{H}), 4.81(\mathrm{~s}, 1 \mathrm{H}), 4.56(\mathrm{~s}$, $1 \mathrm{H}), 2.95(\mathrm{~d}, \mathrm{~J}=13.3 \mathrm{~Hz}, 1 \mathrm{H}), 2.51(\mathrm{dd}, \mathrm{J}=13.2,3.4 \mathrm{~Hz}, 1 \mathrm{H}), 2.43\left(\mathrm{~d}, \mathrm{~J}_{\mathrm{AB}}=15.5 \mathrm{~Hz}\right.$, $1 \mathrm{H}), 2.32\left(\mathrm{~d}, \mathrm{~J}_{\mathrm{AB}}=15.7 \mathrm{~Hz}, 1 \mathrm{H}\right), 2.16(\mathrm{~d}, \mathrm{~J}=10.9 \mathrm{~Hz}, 1 \mathrm{H}), 2.10(\mathrm{dd}, \mathrm{J}=13.3,13.3 \mathrm{~Hz}$, $1 \mathrm{H}), 1.91(\mathrm{~s}, 3 \mathrm{H}), 1.76-1.74(\mathrm{~m}, 1 \mathrm{H}), 1.65-1.61(\mathrm{~m}, 3 \mathrm{H}), 1.54-1.46(\mathrm{~m}, 1 \mathrm{H}), 1.07-1.02$ $(\mathrm{m}, 1 \mathrm{H}), 1.05(\mathrm{~s}, 3 \mathrm{H}), 0.93-0.85(\mathrm{~m}, 1 \mathrm{H}), 0.88(\mathrm{~d}, \mathrm{~J}=6.3 \mathrm{~Hz}, 3 \mathrm{H}), 0.10(\mathrm{~s}, 9 \mathrm{H}) ;{ }^{13} \mathrm{C} \mathrm{NMR}$ $\left(\mathrm{CDCl}_{3}, 500 \mathrm{MHz}\right)$ : 199.64, 163.84, 148.17, 125.76, 108.19, 82.86, 47.19, 46.46, 43.78, 43.54, 38.44, 34.12, 34.02, 27.94, 24.52, 22.39, 22.18, 15.99, 2.89 (3C); IR (neat): 2952 (m), 2925 (m), 2865 (w), 1673 (w), 1454 (m), 1473 (m), $1251(\mathrm{~m}), 1146(\mathrm{~m}), 1105$ (m), $1056(\mathrm{~m}), 889(\mathrm{w}), 837(\mathrm{~s})$; HRMS (EI) $\mathrm{m} / \mathrm{z}$ : calculated 346.2328 for $[\mathrm{M}]^{+}$, found $346.2332 ; \mathrm{mp}=132.2-134.8^{\circ} \mathrm{C}$.

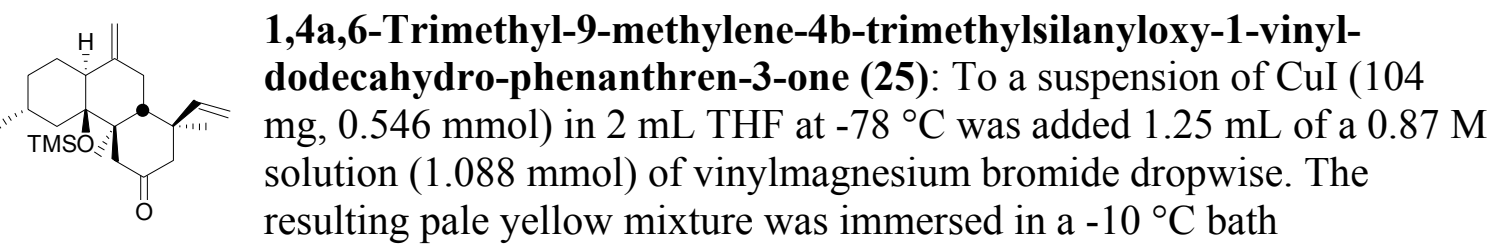

(acetone/ice) for ten minutes. The now olive green suspension was recooled to $-78{ }^{\circ} \mathrm{C}$ for 10 minutes, and enone $24(60 \mathrm{mg}, 0.1731 \mathrm{mmol})$ was cannulated into the reaction mixture using $1 \mathrm{~mL} \mathrm{THF}$. After 1 hour at $-78{ }^{\circ} \mathrm{C}$ the reaction, now rust brown in colour, was quenched with a saturated solution of aqueous $\mathrm{NH}_{4} \mathrm{Cl}$ and warmed to room temperature. The aqueous phase was extracted three times with EtOAc and the combined organic layers dried with $\mathrm{MgSO}_{4}$, filtered and concentrated. Purification by silica gel flash chromatography (5\% EtOAc/hexanes) yielded $52.2 \mathrm{mg}$ of a white solid $(80 \%) .{ }^{1} \mathrm{H}$ $\operatorname{NMR}\left(\mathrm{C}_{6} \mathrm{D}_{6}, 500 \mathrm{MHz}\right): 5.68(\mathrm{dd}, \mathrm{J}=17.3,10.7 \mathrm{~Hz}, 1 \mathrm{H}), 4.93(\mathrm{~d}, \mathrm{~J}=10.7 \mathrm{~Hz}, 1 \mathrm{H}), 4.87$ $(\mathrm{d}, \mathrm{J}=17.5 \mathrm{~Hz}, 1 \mathrm{H}), 4.85-4.84(\mathrm{~m}, 1 \mathrm{H}), 4.64-4.63(\mathrm{~m}, 1 \mathrm{H}), 2.43-2.37(\mathrm{~m}, 2 \mathrm{H}), 2.31-2.25$ $(\mathrm{m}, 3 \mathrm{H}), 2.17(\mathrm{dd}, \mathrm{J}=12.7,2.2 \mathrm{~Hz}, 1 \mathrm{H}), 1.94(\mathrm{dd}, \mathrm{J}=13.9,12.9 \mathrm{~Hz}, 1 \mathrm{H}), 1.83(\mathrm{~d}, \mathrm{~J}=$ $10.7 \mathrm{~Hz}, 1 \mathrm{H}), 1.65-1.50(\mathrm{~m}, 4 \mathrm{H}), 1.34-1.32(\mathrm{~m}, 1 \mathrm{H}), 0.98(\mathrm{~s}, 3 \mathrm{H}), 0.94(\mathrm{~s}, 3 \mathrm{H}), 0.92-0.87$ $(\mathrm{m}, 1 \mathrm{H}), 0.81(\mathrm{~d}, \mathrm{~J}=6.5 \mathrm{~Hz}, 1 \mathrm{H}), 0.75-0.67(\mathrm{~m}, 1 \mathrm{H}), 0.14(\mathrm{~s}, 9 \mathrm{H}) ;{ }^{13} \mathrm{C} \mathrm{NMR}\left(\mathrm{C}_{6} \mathrm{D}_{6}, 500\right.$ MHz): 209.01, 149.29, 149.23, 111.69, 107.22, 84.55, 54.46, 48.56, 48.44, 44.78, 44.46, $43.87,38.10,34.13,33.43,28.48,25.25,22.24,18.50,18.05,2.97$ (3C); IR (neat): 3081 (w), $2947(\mathrm{~m}), 2928(\mathrm{~m}), 2871(\mathrm{w}), 2851(\mathrm{w}), 1709(\mathrm{~s}), 1642(\mathrm{w}), 1448(\mathrm{w}), 1279(\mathrm{~m})$, 1261 (m), 1247 (s), 1123 (m), 1059 (m), 912 (w), 834 (s); HRMS (EI) m/z: calculated 374.2641 for $[\mathrm{M}]^{+}$, found $374.263, \mathrm{mp}=112.2-113.0^{\circ} \mathrm{C}$. 


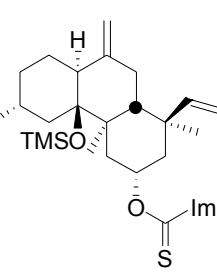

Imidazole-1-carbothioic acid 0-(1,4a,6-trimethyl-9-methylene-4btrimethylsilanyloxy-1-vinyl-tetradecahydro-phenanthren-3-yl) ester (27): A solution of $25(21.1 \mathrm{mg}, 0.056 \mathrm{mmol})$ in $2 \mathrm{~mL}$ of THF was cooled to $-78^{\circ} \mathrm{C}$. DIBAL-H $(0.07 \mathrm{~mL}$ of a $1.0 \mathrm{M}$ solution in toluene, $0.105 \mathrm{mmol}$ ) was added dropwise and the reaction allowed to warm to 0

${ }^{\circ} \mathrm{C}$. The reaction was quenched with a few drops of acetone followed by a

1:1 solution of $1 \mathrm{M} \mathrm{NaOH}$ (aq.) and $1 \mathrm{M}$ sodium tartrate (aq.) and stirred vigorously for 1 hour. After extracting the aqueous phase three times with EtOAc, drying over $\mathrm{MgSO}_{4}$, filtering and concentrating, the crude product was dissolved in $3 \mathrm{~mL} \mathrm{DCM}$.

Thiocarbonyldiimidazole (34 $\mathrm{mg}$ of $90 \%$ pure, $0.172 \mathrm{mmol}$ ) followed by DMAP ( $7 \mathrm{mg}$, $0.057 \mathrm{mmol}$ ) were added. The reaction was stirred at ambient temperature for 36 hours and then concentrated. Purification by silica gel flash chromatography (10\% EtOAc/hexanes) yielded $23 \mathrm{mg}$ of a clear, colourless oil ( $84 \%$ for two steps). ${ }^{1} \mathrm{H}$ NMR $\left(\mathrm{CDCl}_{3}, 500 \mathrm{MHz}\right): 8.35(\mathrm{~s}, 1 \mathrm{H}), 7.64(\mathrm{~s}, 1 \mathrm{H}), 7.03(\mathrm{~s}, 1 \mathrm{H}), 5.96-5.93(\mathrm{~m}, 1 \mathrm{H}), 5.59(\mathrm{dd}, \mathrm{J}$ $=17.5,10.8 \mathrm{~Hz}, 1 \mathrm{H}), 5.01(\mathrm{~d}, \mathrm{~J}=10.8 \mathrm{~Hz}, 1 \mathrm{H}), 4.96(\mathrm{~d}, \mathrm{~J}=17.4 \mathrm{~Hz}, 1 \mathrm{H}), 4.65(\mathrm{~s}, 1 \mathrm{H})$, $4.45(\mathrm{~s}, 1 \mathrm{H}), 2.15-2.02(\mathrm{~m}, 3 \mathrm{H}), 1.99-1.94(\mathrm{~m}, 2 \mathrm{H}), 1.89(\mathrm{dd}, \mathrm{J}=14.7,14.5 \mathrm{~Hz}, 1 \mathrm{H})$, $1.73-1.70(\mathrm{~m}, 1 \mathrm{H}), 1.65(\mathrm{dd}, \mathrm{J}=15.6,3.5 \mathrm{~Hz}, 1 \mathrm{H}), 1.62-1.54(\mathrm{~m}, 3 \mathrm{H}), 1.49-1.41(\mathrm{~m}, 1 \mathrm{H})$, 1.32 (s, 3H), 1.26-1.24 (m, 1H), 1.19 (s, 3H), 1.11 (dd, J = 12.6, 13.1 Hz, 1H), 0.90-0.83 $(\mathrm{m}, 1 \mathrm{H}), 0.85(\mathrm{~d}, \mathrm{~J}=6.4 \mathrm{~Hz}, 3 \mathrm{H}), 0.13(\mathrm{~s}, 9 \mathrm{H}) ;{ }^{13} \mathrm{C} \mathrm{NMR}\left(\mathrm{CDCl}_{3}, 500 \mathrm{MHz}\right): 183.48$, $150.16,149.57,136.91,130.88,118.02,111.83,106.61,84.73,81.34,43.36,43.07$, 41.86, 40.96, 38.51, 38.09, 34.70, 34.02, 32.93, 28.16, 24.79, 22.25, 19.58, 19.30, 3.05 (3C); IR (neat): 3085 (w), 2952 (s), 2926 (s), 2869 (m), 1728 (w), 1645 (m), 1573 (w), 1531 (w), 1458 (m), 1386 (s), 1327 (s), 1281 (s), 1247 (s), 1232 (s), 1188 (s), 1113 (s), 1092 (s), 1061 (m), 971 (s), 836 (s); HRMS (EI) m/z: calculated 330.2378 for [M-OTMS$[\mathrm{Im}]^{+}$, found 330.2017 .

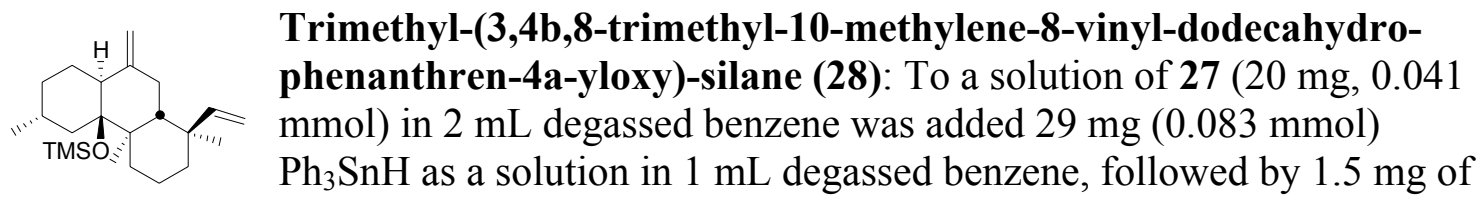

AIBN (0.009 mmol). The reaction mixture was brought to reflux and allowed to react for 1 hour 15 minutes. After cooling the room temperature and reaction was concentrated and purified by silica gel flash chromatography (100\% hexanes) to give $11 \mathrm{mg}$ of a clear colourless oil (74\%). ${ }^{1} \mathrm{H}$ NMR $\left(\mathrm{CDCl}_{3}, 500 \mathrm{MHz}\right): 5.58(\mathrm{dd}, \mathrm{J}=17.5,10.8 \mathrm{~Hz}, 1 \mathrm{H}), 4.90$ $(\mathrm{d}, \mathrm{J}=10.8 \mathrm{~Hz}, 1 \mathrm{H}), 4.86(\mathrm{~d}, \mathrm{~J}=17.8 \mathrm{~Hz}, 1 \mathrm{H}), 4.59$ (s, 1H), 4.39 (s, 1H), 2.06-1.92 (m, $3 \mathrm{H}), 1.79(\mathrm{dd}, \mathrm{J}=12.8,3.4 \mathrm{~Hz}, 1 \mathrm{H}), 1.70-1.67(\mathrm{~m}, 1 \mathrm{H}), 1.63-1.50(5 \mathrm{H}), 1.48-1.41(\mathrm{~m}$, $2 \mathrm{H}), 1.35(\mathrm{dd}, \mathrm{J}=13.0,3.8 \mathrm{~Hz}, 1 \mathrm{H}), 1.31-1.20(\mathrm{~m}, 2 \mathrm{H}), 1.10-1.05(\mathrm{~m}, 1 \mathrm{H}), 1.08(\mathrm{~s}, 3 \mathrm{H})$, $0.98(\mathrm{~s}, 3 \mathrm{H}), 0.88-0.80(\mathrm{~m}, 1 \mathrm{H}), 0.85(\mathrm{~d}, \mathrm{~J}=6.4 \mathrm{~Hz}, 3 \mathrm{H}), 0.12(\mathrm{~s}, 9 \mathrm{H}) ;{ }^{13} \mathrm{C} \mathrm{NMR}\left(\mathrm{CDCl}_{3}\right.$, $500 \mathrm{MHz}$ ): 151.95, 150.80, 110.36, 105.73, 84.62, 44.23, 43.66, 42.43, 39.87, 39.68, 37.90, 34.24, 33.38, 31.90, 28.22, 24.93, 22.36, 18.50, 17.61, 17.22, 2.99 (3C); IR (neat): 3085 (w), 2949 (s), 2869 (m), 1645 (m), 1455 (m), 1246 (s), 1181 (m), 1124 (s), 1090 (s), 1059 (s), 907 (m), 881 (m), 831 (s), 748 (m); HRMS (EI) m/z: calculated 360.2848 for $[\mathrm{M}]^{+}$, found 360.2825 . 


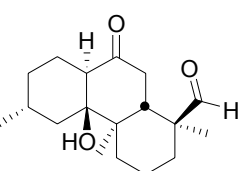

4b-Hydroxy-1,4a,6-trimethyl-9-oxo-tetradecahydro-phenanthrene-1carbaldehyde (29): To a solution of $\mathbf{2 8}(34 \mathrm{mg}, 0.094 \mathrm{mmol})$ in $2 \mathrm{~mL}$ THF was added $0.28 \mathrm{~mL}$ of a $1.0 \mathrm{M}$ solution of TBAF $(0.280 \mathrm{mmol})$. After 5 hours at room temperature the reaction was quenched with a saturated solution of aqueous $\mathrm{NH}_{4} \mathrm{Cl}$ and the aqueous phase extracted three times with EtOAc. The combined organic layers were dried with $\mathrm{MgSO}_{4}$, filtered and concentrated. Further purification was not required and the crude material was dissolved in $4 \mathrm{~mL}$ of 5:1 THF $/ \mathrm{H}_{2} \mathrm{O}$. To this solution was added $0.06 \mathrm{~mL}$ of $4 \%$ aqueous solution of $\mathrm{OsO}_{4}$ $(0.009 \mathrm{mmol})$ followed by $44 \mathrm{mg}$ of NMO $(0.376 \mathrm{mmol})$. After 4 hours of stirring at room temperature, $121 \mathrm{mg}(0.566 \mathrm{mmol})$ of $\mathrm{NaIO}_{4}$ was added and reaction was allowed to proceed for another 10 hours. An aqueous solution of $\mathrm{Na}_{2} \mathrm{SO}_{3}$ was added and the biphasic mixture stirred vigorously for 30 minutes. The aqueous phase was extracted three times with diethyl ether and the combined organic phases dried over $\mathrm{MgSO}_{4}$, filtered and concentrated. Purification by silica gel flash chromatography $(30 \%$ EtOAc/hexanes) yielded $23 \mathrm{mg}$ of a white solid ( $83 \%$ for two steps). ${ }^{1} \mathrm{H} \mathrm{NMR}\left(\mathrm{CDCl}_{3}\right.$, $500 \mathrm{MHz}): 9.19(\mathrm{~s}, 1 \mathrm{H}), 2.72(\mathrm{dd}, \mathrm{J}=14.1,3.4 \mathrm{~Hz}, 1 \mathrm{H}), 2.39(\mathrm{dd}, \mathrm{J}=12.0,4.1 \mathrm{~Hz}, 1 \mathrm{H})$, $2.31(\mathrm{dd}, \mathrm{J}=14.2,14.2 \mathrm{~Hz}, 1 \mathrm{H}), 1.85-1.79(\mathrm{~m}, 2 \mathrm{H}), 1.76-1.64(\mathrm{~m}, 3 \mathrm{H}), 1.62-1.54(\mathrm{~m}$, $6 \mathrm{H}), 1.50-1.42(\mathrm{~m}, 1 \mathrm{H}), 1.30-1.21(\mathrm{~m}, 2 \mathrm{H}), 1.23(\mathrm{~s}, 3 \mathrm{H}), 1.11(\mathrm{~s}, 3 \mathrm{H}), 0.91(\mathrm{~d}, \mathrm{~J}=6.4 \mathrm{~Hz}$, $3 \mathrm{H}$ ), 0.79 (dddd, $\mathrm{J}=12.8,12.8,12.8,3.6 \mathrm{~Hz}, 1 \mathrm{H}), ;{ }^{13} \mathrm{C} \mathrm{NMR}\left(\mathrm{CDCl}_{3}, 500 \mathrm{MHz}\right)$ : 209.56, 205.02, 80.17, 51.77, 49.43, 40.86, 40.62, 38.73, 38.48, 32.91, 31.93, 30.37, 27.74, 22.36, 21.13, 17.03, 16.49, 13.64; IR (neat): 3514 (br, w), 2946 (s), 2868 (w), $1712(\mathrm{~s}), 1456(\mathrm{~m}), 1374(\mathrm{~m}), 1315(\mathrm{w}), 1247(\mathrm{w}), 1156(\mathrm{w}), 1066(\mathrm{w}), 1023(\mathrm{w}), 946$ (w), $880(w), 736(w)$; HRMS (EI) m/z: calculated 292.2038 for [M] $]^{+}$, found 292.2054; $\mathrm{mp}=117.3-119.6^{\circ} \mathrm{C}$.

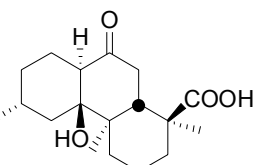
4b-Hydroxy-1,4a,6-trimethyl-9-oxo-tetradecahydro-phenanthrene- 1-carboxylic acid (30): To a solution of $\mathbf{2 9}(12.5 \mathrm{mg}, 0.041 \mathrm{mmol})$ in $1.0 \mathrm{~mL}$ of a $3: 1 \mathrm{tBuOH}: \mathrm{H}_{2} \mathrm{O}$ solution was added $49 \mathrm{mg}(0.408 \mathrm{mmol})$ $\mathrm{NaH}_{2} \mathrm{PO}_{4}$ followed by $0.36 \mathrm{~mL}(4.075 \mathrm{mmol})$ cyclopentene. The reaction was cooled to $0{ }^{\circ} \mathrm{C}$ and $22 \mathrm{mg}(0.243 \mathrm{mmol}) \mathrm{NaClO}_{2}$ in $0.5 \mathrm{~mL}$ of $3: 1$ tBuOH: $\mathrm{H}_{2} \mathrm{O}$ added. After 15 minutes the reaction was quenched with a saturated aqueous solution of $\mathrm{Na}_{2} \mathrm{SO}_{3}$ and brine. The aqueous phase was extracted three times with EtOAc and the combined organic phases dried over $\mathrm{MgSO}_{4}$, filtered and concentrated. Purification by silica gel flash chromatography ( $30 \%$ EtOAc/hexanes and $1 \% \mathrm{AcOH})$ yielded $9.2 \mathrm{mg}(78 \%)$ of a white solid. ${ }^{1} \mathrm{H}$ NMR $\left(\mathrm{CDCl}_{3}, 300 \mathrm{MHz}\right): 2.92$ (dd, J = 13.8, $3.0 \mathrm{~Hz}, 1 \mathrm{H}), 2.34$ (dd, J = 11.9, $3.8 \mathrm{~Hz}, 1 \mathrm{H}), 2.33$ (dd, J = 14.4, $13.4 \mathrm{~Hz}, 1 \mathrm{H}), 2.09$ (dd, J $=14.4,3.2 \mathrm{~Hz}, 1 \mathrm{H}), 1.82-1.75(\mathrm{~m}, 2 \mathrm{H}), 1.68-1.47(\mathrm{~m}, 9 \mathrm{H}), 1.25-1.17(\mathrm{~m}, 1 \mathrm{H}), 1.21(\mathrm{~s}$, $3 \mathrm{H}), 1.18(\mathrm{~s}, 3 \mathrm{H}), 0.88(\mathrm{~d}, \mathrm{~J}=6.1 \mathrm{~Hz}, 3 \mathrm{H}), 0.82-0.69(\mathrm{~m}, 1 \mathrm{H}), 2$ exchangeable protons do not appear; ${ }^{1} \mathrm{H}$ NMR $\left(\mathrm{CD}_{3} \mathrm{OD}, 500 \mathrm{MHz}\right): 3.01(\mathrm{dd}, \mathrm{J}=14.0,3.4 \mathrm{~Hz}, 1 \mathrm{H}), 2.48(\mathrm{dd}, \mathrm{J}=$ 13.9, $14.0 \mathrm{~Hz}, 1 \mathrm{H}), 2.47$ (dd, J = 10.9, $4.6 \mathrm{~Hz}, 1 \mathrm{H}), 1.97$ (dd, J = 14.5, $3.3 \mathrm{~Hz}, 1 \mathrm{H}), 1.84-$ $1.75(\mathrm{~m}, 1 \mathrm{H}), 1.74-1.58(\mathrm{~m}, 9 \mathrm{H}), 1.52-1.49(\mathrm{~m}, 1 \mathrm{H}), 1.31-1.26(\mathrm{~m}, 1 \mathrm{H}), 1.28(\mathrm{~s}, 3 \mathrm{H}), 1.26$ $(\mathrm{s}, 3 \mathrm{H}), 0.91(\mathrm{~d}, \mathrm{~J}=6.4 \mathrm{~Hz}, 3 \mathrm{H}), 0.89-0.79(\mathrm{~m}, 1 \mathrm{H}), 2$ exchangeable protons do not appear; ${ }^{13} \mathrm{C} \mathrm{NMR}\left(\mathrm{CDCl}_{3}, 300 \mathrm{MHz}\right): 211.86,182.69,80.58,52.09,47.10,41.54,41.14$, $40.80,38.98,36.55,33.35,30.72,27.98,22.78,21.47,18.18,16.88,16.44 ;{ }^{13} \mathrm{C}$ NMR ( $\left.\mathrm{CD}_{3} \mathrm{OD}, 500 \mathrm{MHz}\right): 212.72,180.28,79.71,51.43,46.48,41.19,40.41,40.31,37.95$, 
36.22, 32.91, 30.15, 27.02, 21.35, 20.81, 17.49, 15.51, 15.26; IR (neat): 3526 (m, br), 2952 (s), 2873 (m), 1698 (s), 1454 (m), 1390 (m), 1367 (m), 1268 (m), 1242 (s), 1151 (s), $1128(\mathrm{~m}), 736(\mathrm{~m})$; HRMS (EI) m/z: calculated 308.1988 for $[\mathrm{M}]^{+}$, found 308.1974; $\mathrm{mp}=$ 178.6- $180.9^{\circ} \mathrm{C}$.

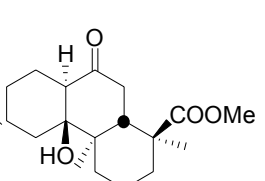

4b-Hydroxy-1,4a,6,8a-tetramethyl-9-oxo-tetradecahydrophenanthrene-1-carboxylic acid methyl ester (31): To a solution of $30(4.5 \mathrm{mg}, 0.015 \mathrm{mmol})$ in $0.5 \mathrm{~mL}$ ether cooled to $0{ }^{\circ} \mathrm{C}$ was added a solution of diazomethane in ether dropwise until a faint yellow colour persisted. A small amount of silica gel $(0.2 \mathrm{~mL})$ was added and the reaction mixture was concentrated. Purification by silica gel flash chromatography (20\% EtOAc/hexanes) yielded $4.4 \mathrm{mg}$ of a white solid $(94 \%) .{ }^{1} \mathrm{H} \mathrm{NMR}\left(\mathrm{CDCl}_{3}, 300 \mathrm{MHz}\right)$ : $3.63(\mathrm{~s}, 3 \mathrm{H}), 2.93(\mathrm{dd}, \mathrm{J}=14.0,3.5 \mathrm{~Hz}, 1 \mathrm{H}), 2.37-2.28(\mathrm{~m}, 2 \mathrm{H}), 1.93(\mathrm{dd}, \mathrm{J}=14.3,3.5$ $\mathrm{Hz}, 1 \mathrm{H}), 1.85-1.69(\mathrm{~m}, 3 \mathrm{H}), 1.67-1.40(\mathrm{~m}, 9 \mathrm{H}), 1.27-1.19(\mathrm{~m}, 1 \mathrm{H}), 1.23(\mathrm{~s}, 3 \mathrm{H}), 1.19(\mathrm{~s}$, $3 \mathrm{H}), 0.90(\mathrm{~d}, \mathrm{~J}=6.2 \mathrm{~Hz}, 3 \mathrm{H}), 0.86-0.70(\mathrm{~m}, 1 \mathrm{H}) ;{ }^{13} \mathrm{C} \mathrm{NMR}\left(\mathrm{CDCl}_{3}, 500 \mathrm{MHz}\right): 210.20$, 178.07, 80.02, 52.19, 51.65, 47.17, 41.27, 40.98, 40.60, 38.73, 36.25, 33.01, 30.45, 27.75, 22.39, 21.20, 17.88, 16.53, 16.38; IR (neat): 3522 (m, br), 2948 (s), 2867 (m), 1710 (s), 1456 (m), 1391 (w), 1372 (w), 1255 (m), 1241 (m), 1197 (w), 1152 (m), 1092 (w);

HRMS (EI) m/z: calculated 322.2144 for $[\mathrm{M}]^{+}$, found $322.2135 ; \mathrm{mp}=186.3-191.6{ }^{\circ} \mathrm{C}$. 


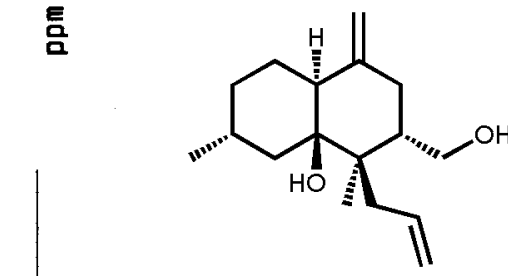

Current Data Parameters

$$
\begin{aligned}
& \text { NAME } \\
& \text { EXPNO }
\end{aligned}
$$

PAOCNO

effie_134

F2 - Acquisition Parame

Date 20030212

13.05

PULPROG

SOLVENT COC13

AQ

FIO

1.0485960

DW

AG

MUCLEUS

0.476837
16.0

D11

P31

S2

HLI

P1

DE

SF01

SWH

TD

NS

F1 - Processing paramet SI 32768

SF $\quad 125.7591504$

SSB

LB

GB

EM

1.00
0

10 NMR plot parameters

CX

FI

F1

F2

PPMCM

$\mathrm{HZCM}$

$$
\begin{array}{r}
220.000 \\
27667.01 \\
-10.000 \\
-1257.59 \\
10.45455 \\
314.75476
\end{array}
$$

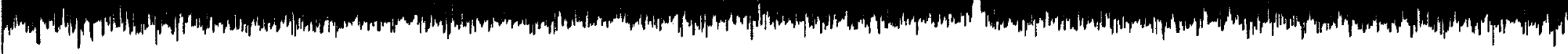



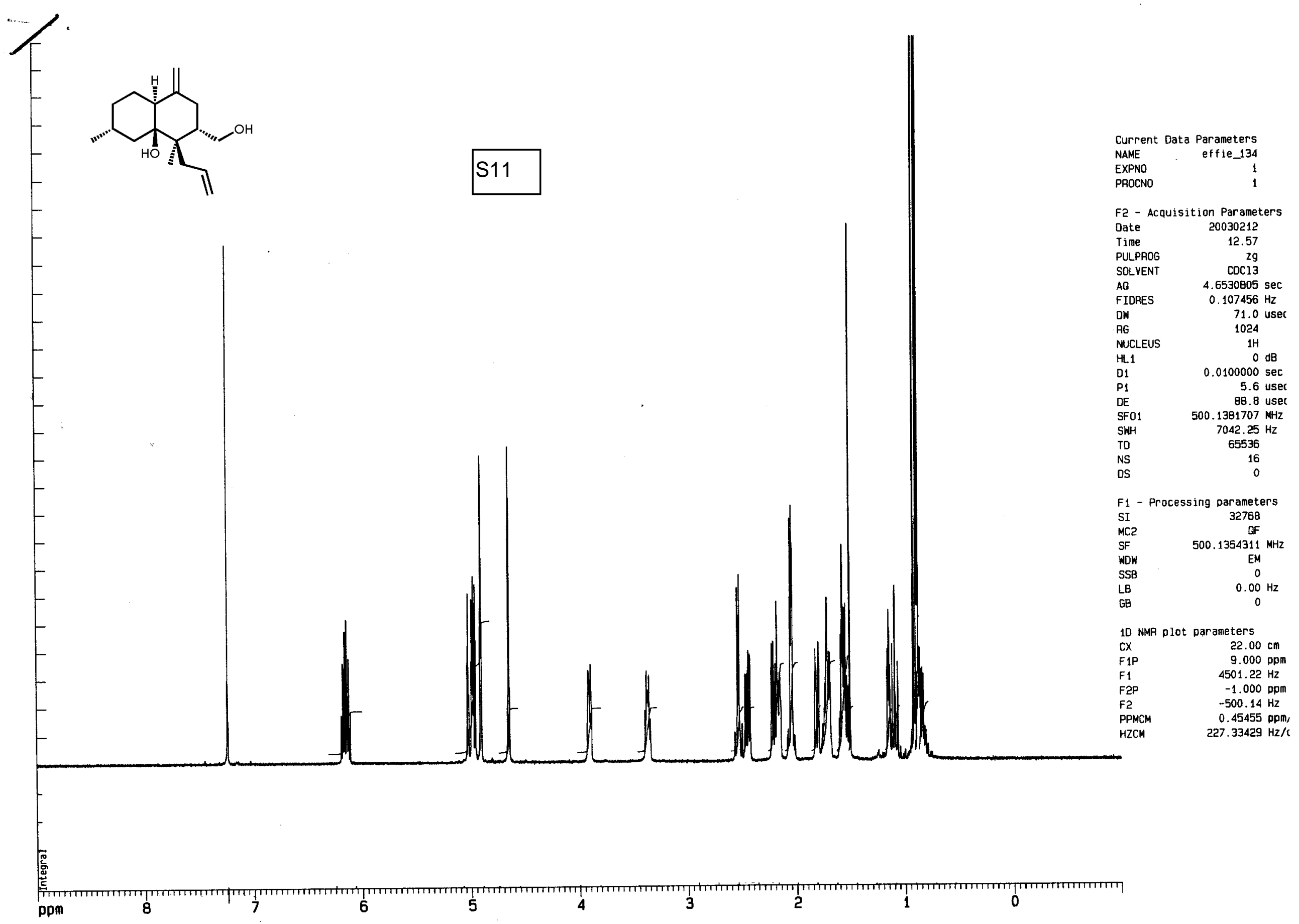


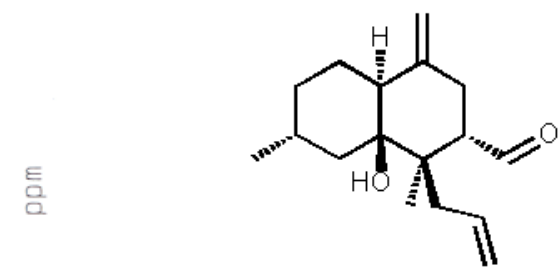

13C with oroton decounlino

\section{S12}

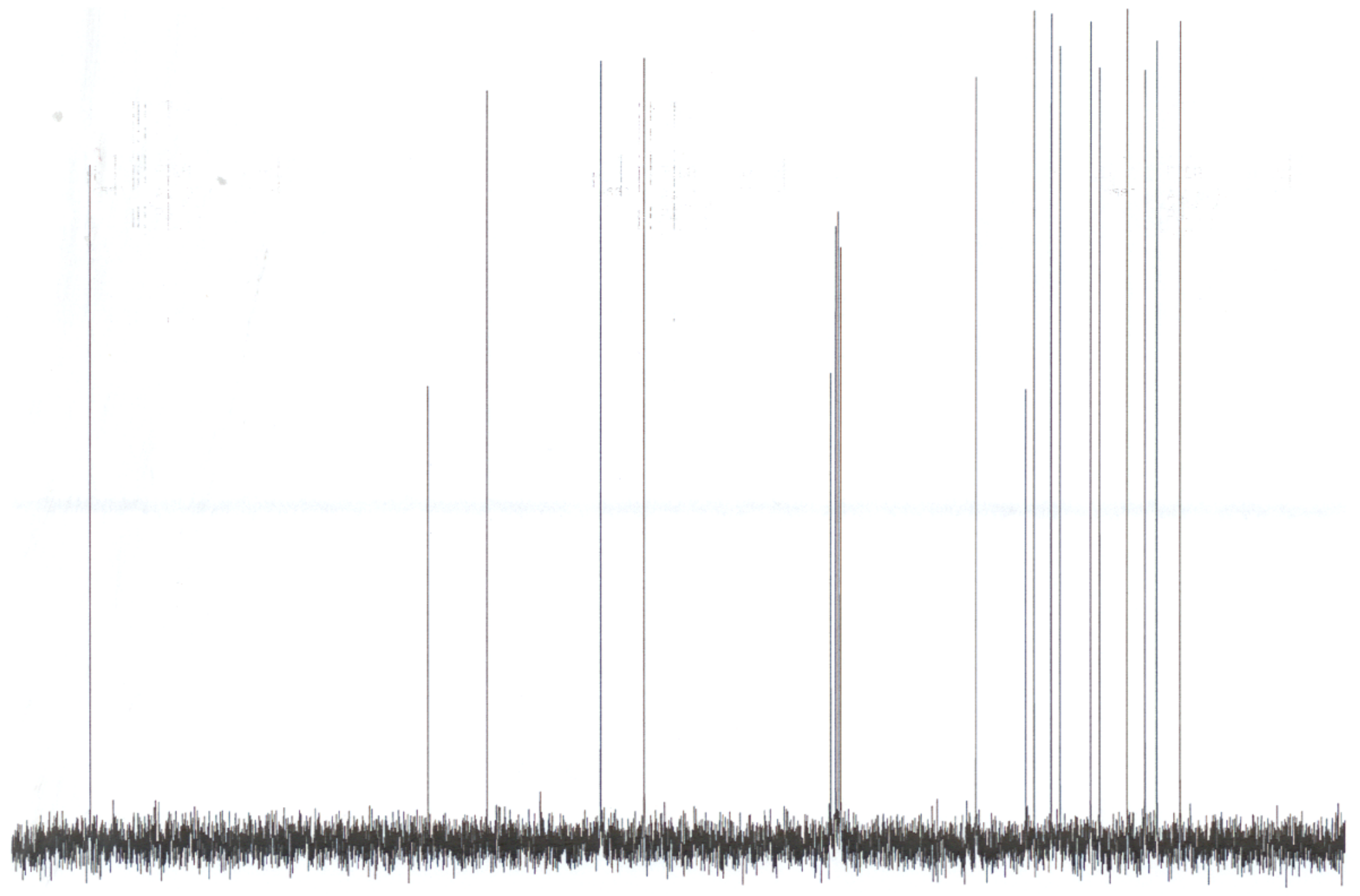

Nil

ppm 200

175

150

125

100

75

50

25

0

PPZCN

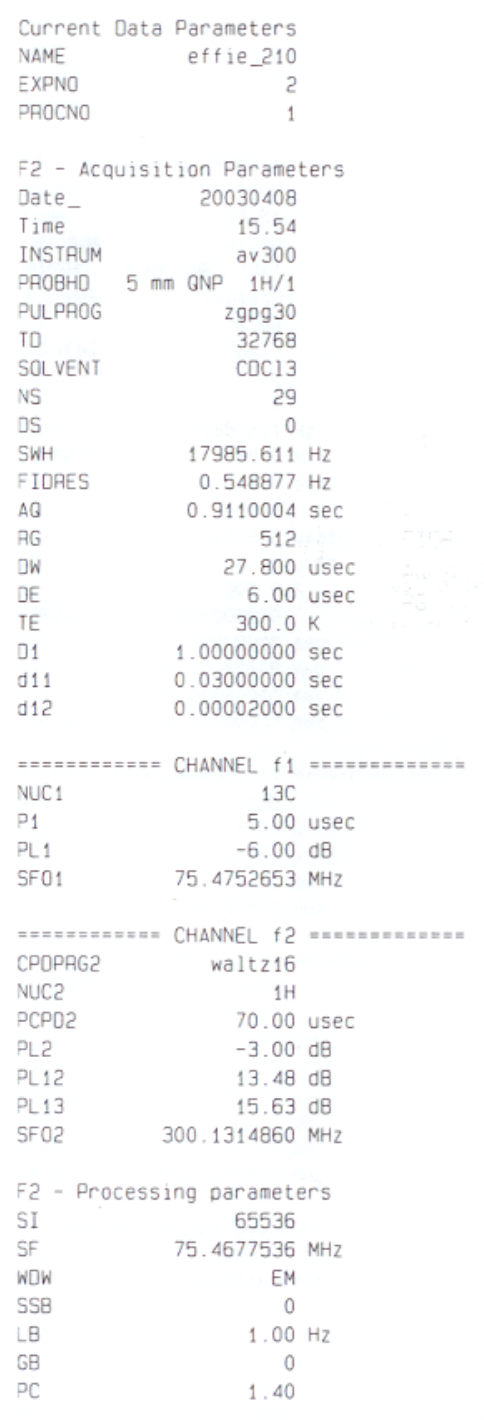

10 NMP plot parameters

$\begin{array}{lc}C X & 20.00 \mathrm{~cm} \\ C Y & 12.50 \mathrm{~cm} \\ \text { F1P } & 220.000 \mathrm{ppm} \\ \text { F1 } & 16602.90 \mathrm{~Hz} \\ \text { F2P } & -10.000 \mathrm{ppm} \\ \text { F2 } & -754.68 \mathrm{~Hz} \\ \text { PPMCM } & 11.50000 \mathrm{ppm} / \mathrm{cm} \\ \text { HZCM } & 867.87909 \mathrm{~Hz} / \mathrm{cm}\end{array}$




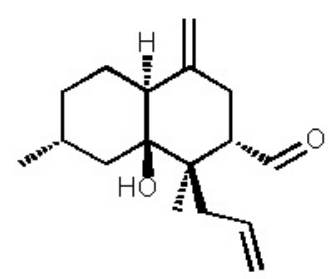

Current Data Parameters

NAME effie_210

PROCNO

F2 - Acquisition Parameters

Date_ 20030408

Time $\quad 15.51$

INSTRUM

PULPAOG

TO 30720

SOLVENT CDC13

DS 0

SWH $5081.301 \mathrm{~Hz}$

$\begin{array}{lr}\text { FIDRES } & 0.165407 \mathrm{~Hz} \\ A Q & 3.0228980 \mathrm{sec}\end{array}$

RG $\quad 45.3$

$\begin{array}{lr}\text { DW } & 98.400 \text { usec } \\ \text { DE } & 6.00 \text { usec }\end{array}$

TE $300.0 \mathrm{~K}$

$01 \quad 1.00000000 \mathrm{sec}$

$=z=z=z=z=z=$ CHANNEL $f 1$ $=z=z=z=z=z=*$

NUC1 $1 \mathrm{H}$

P1 10.50 usec

PL1 $\quad-3.00 \mathrm{~dB}$

SFO1 $300 \cdot 1319477 \mathrm{MHz}$

F2 - Processing parameters

FI - Processing parameters

$\begin{array}{ll}\text { SI } & 65536 \\ \text { SF } & 300.1300000 \mathrm{MHz}\end{array}$

WOW EM

$\begin{array}{lc}\text { SSB } & 0 \\ \text { LB } & 0.10 \mathrm{HZ}\end{array}$

$\begin{array}{lr}\text { LB } & 0.10 \\ \text { GB } & 0 \\ \text { PC } & 1.00\end{array}$
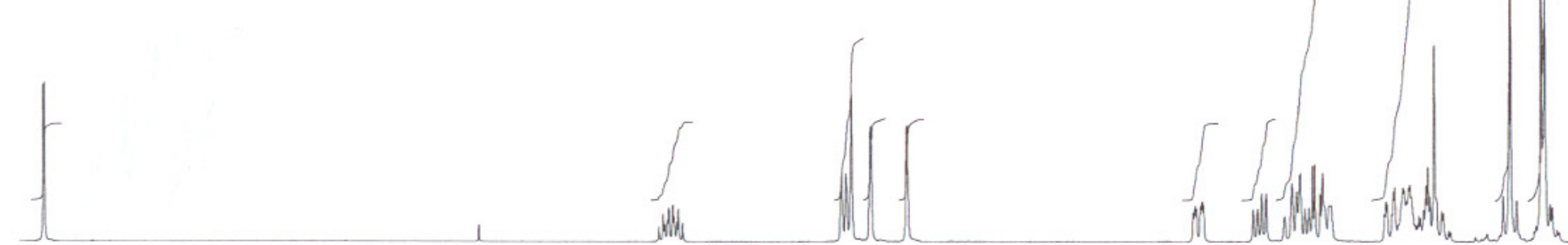

10 NMP plot parameters

CX

CY $\quad 10.00 \mathrm{~cm}$

F1P $\quad 10.000 \mathrm{ppm}$

$\begin{array}{ll}1 & 1001.30 \mathrm{~Hz}\end{array}$

द2P $\quad 0.000 \mathrm{ppm}$

F2 $0.00 \mathrm{~Hz}$

PPMCM $\quad 0.50000 \mathrm{ppm} / \mathrm{cm}$

HZCM $\quad 150.06500 \mathrm{~Hz} / \mathrm{cm}$

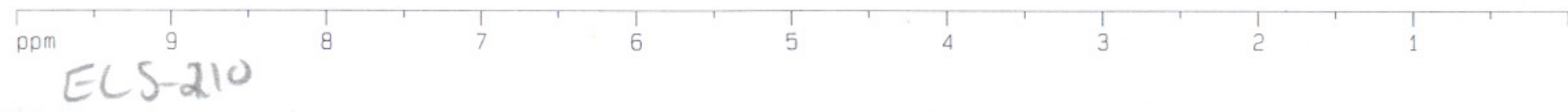


<smiles>C=CC[C@H]1C([C@H](O)C=C)CC(=C)C2CCC(C)CC2(O)[C@H]1C</smiles>

S14

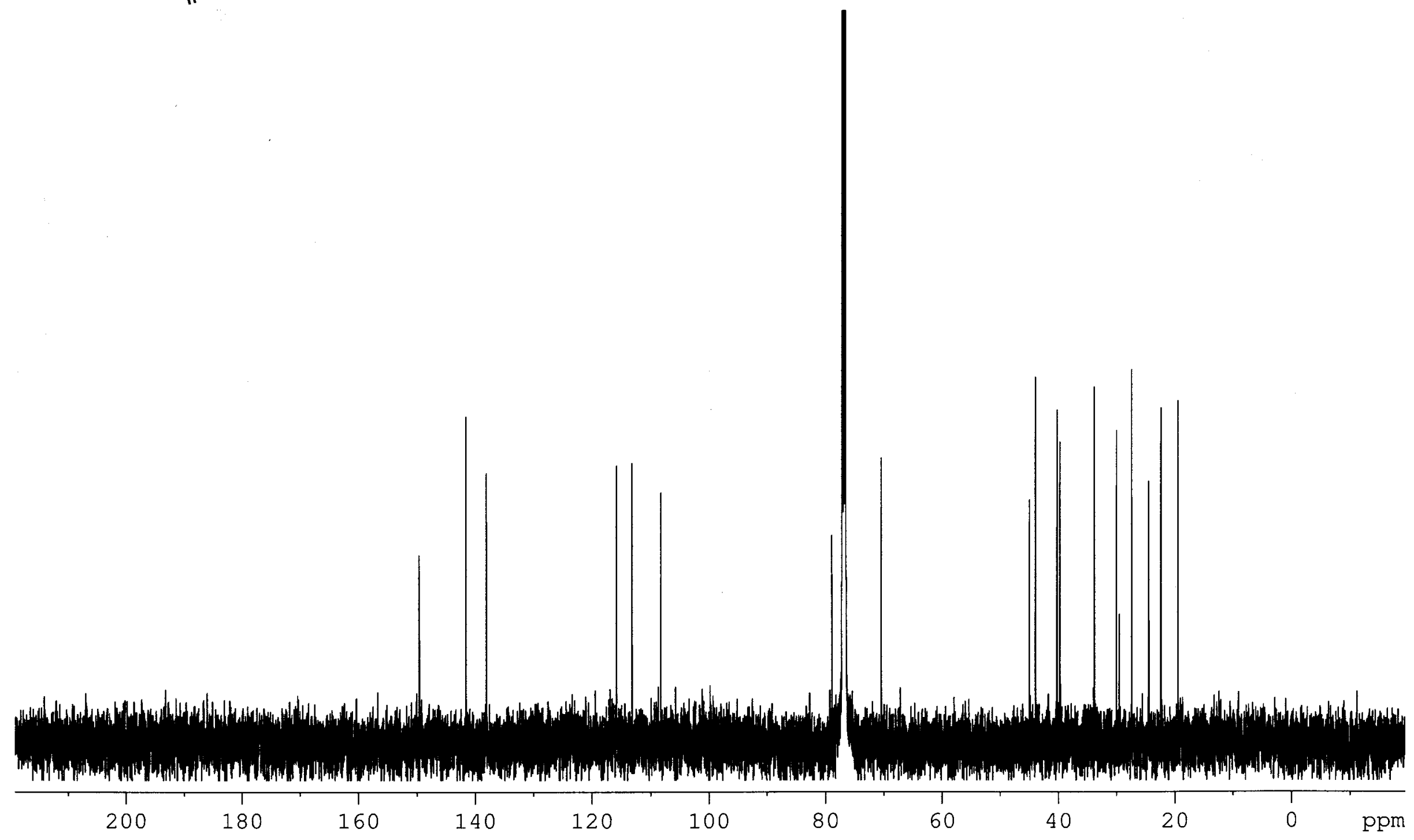




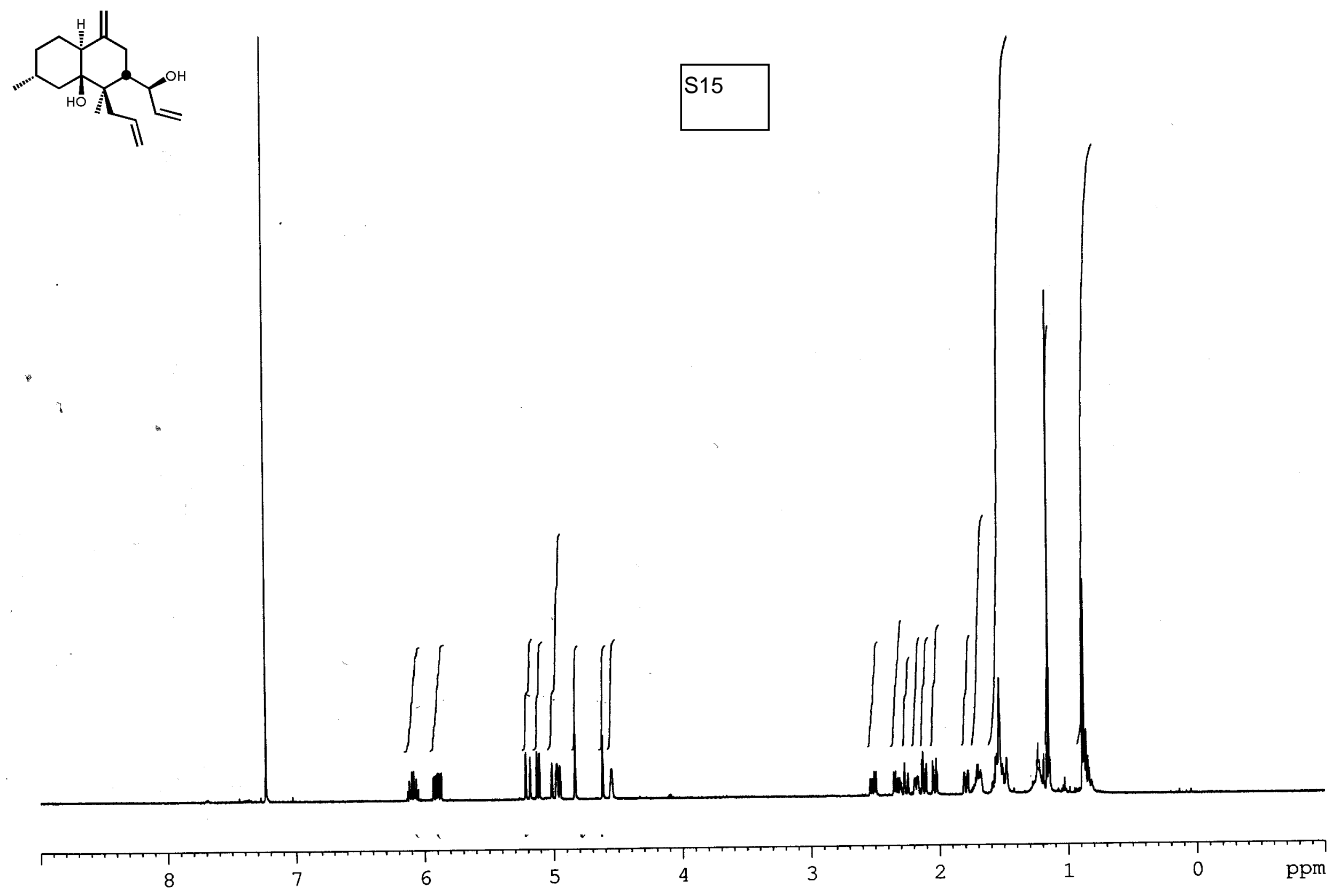




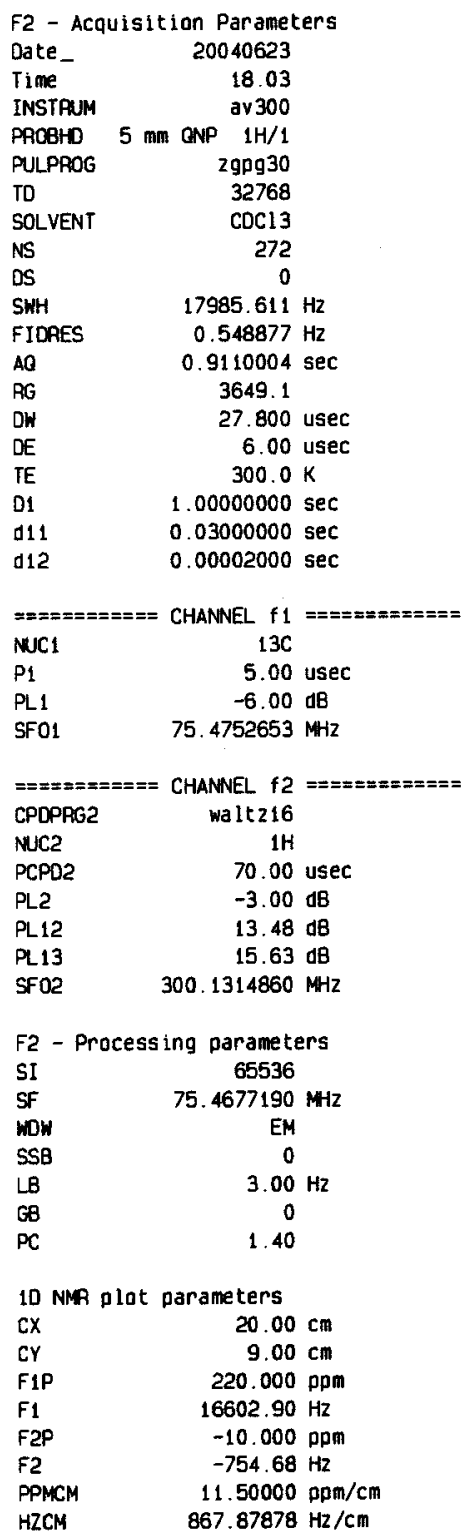




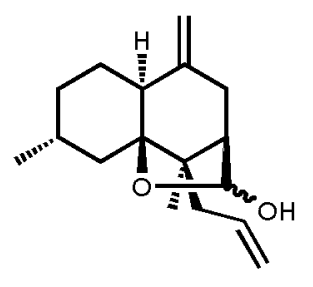

1H NMP

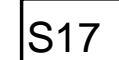

Current Data Parameters

NAME effie_other_isomer

EXPNO

PROCNO

F2 - Acquisition Parameters

Date_ 20040623

Time $\quad 18.01$

INSTRUM av300

PAOBHD 5 MIII ONP $1 H / 1$

$\begin{array}{lr}\text { PULPROG } & 2930 \\ \text { TD } & 30720\end{array}$

SOLVENT CDC13

$\begin{array}{lr}\text { NS } & 16 \\ \text { DS } & 0\end{array}$

SWH $\quad 5001.301 \mathrm{~Hz}$

FIDAES $\quad 0.165407 \mathrm{~Hz}$

A0 $\quad 3.0228980 \mathrm{sec}$

$\begin{array}{lr}\text { AG } & 149.7 \\ \text { DW } & 98.400 \text { usec }\end{array}$

DE 6.00 usec

TI 1.0000000

$01 \quad 1.00000000 \mathrm{sec}$

$=\ldots$

NUCA

$P_{1} \quad 10.50$ usec

PL1 $300.1919477 \mathrm{NH}$

SFO1 $300.1319477 \mathrm{MHz}$

F2 - Processing parameters

SI $\quad 65536$

SF $\quad 300.1300000 \mathrm{MHz}$

WDW

$\begin{array}{lc}\text { SSB } & 0 \\ \text { LB } & 0.10 \mathrm{~Hz} \\ G B & 0\end{array}$

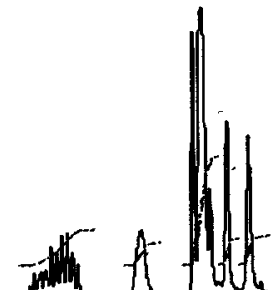

1.00

10 NMP plot parameters

$\begin{array}{lc}\text { CX } & 20.00 \mathrm{~cm} \\ \text { CY } & 10.00 \mathrm{~cm} \\ \text { F1P } & 9.000 \mathrm{ppm} \\ \text { F1 } & 2701.17 \mathrm{~Hz} \\ \text { F2P } & -1.000 \mathrm{ppm} \\ \text { F2 } & -300.13 \mathrm{~Hz} \\ \text { PPMCM } & 0.50000 \mathrm{ppm} / \mathrm{cm} \\ \text { HZCM } & 150.06500 \mathrm{~Hz} / \mathrm{cm}\end{array}$

总 


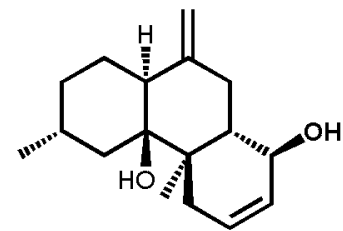

Current Data Parameters

NAME effie_212

PROCNO

F2 - Acquisition Parame Date 20030406

Date

PUPROG 2gdc

COLPROG
SOLVENT

FIDRES

1.0485960

0.476837

DW $\quad 16.0$

AG 32768

NUCLEUS $\quad 13 \mathrm{C}$

$011 \quad 0.0300000$

P31

$\begin{array}{ll}S 2 & 18 \\ \text { HL1 } & 22\end{array}$

$\begin{array}{ll}1 & 1.0000000\end{array}$

P1 2.0

SFO1 $\quad 125.7724464$

$\begin{array}{lr}\text { SWH } & 125.7724464 \\ \text { SWH } & 31250.00\end{array}$

$\begin{array}{lr}\text { SWH } & 31250.00 \\ \text { TD } & 65536\end{array}$

$\begin{array}{lr}\text { NS } & 1926 \\ \text { DS } & 0\end{array}$

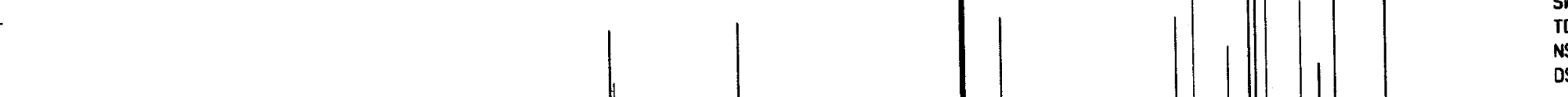

F1 - Processing paramet SI 32768 $\begin{array}{lr}\text { SI } & \text { OF } \\ \text { MC2 } & \end{array}$ $\begin{array}{lr}\text { MC2 } & \text { OF } \\ \text { SF } & 125.7591485\end{array}$ WOW

SSB

LB $\mathrm{CX} 22.00$ F1P $\quad 220.000$ F1 27667.01

F2P -10.000

F2 -1257.59

PPMCM $\quad 10.45455$

$\begin{array}{lr}\text { PPMCM } & 10.45455 \\ \text { HZCM } & 1314.75476\end{array}$
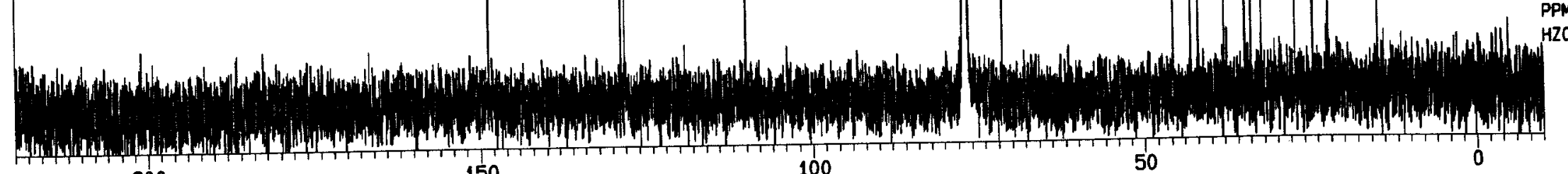


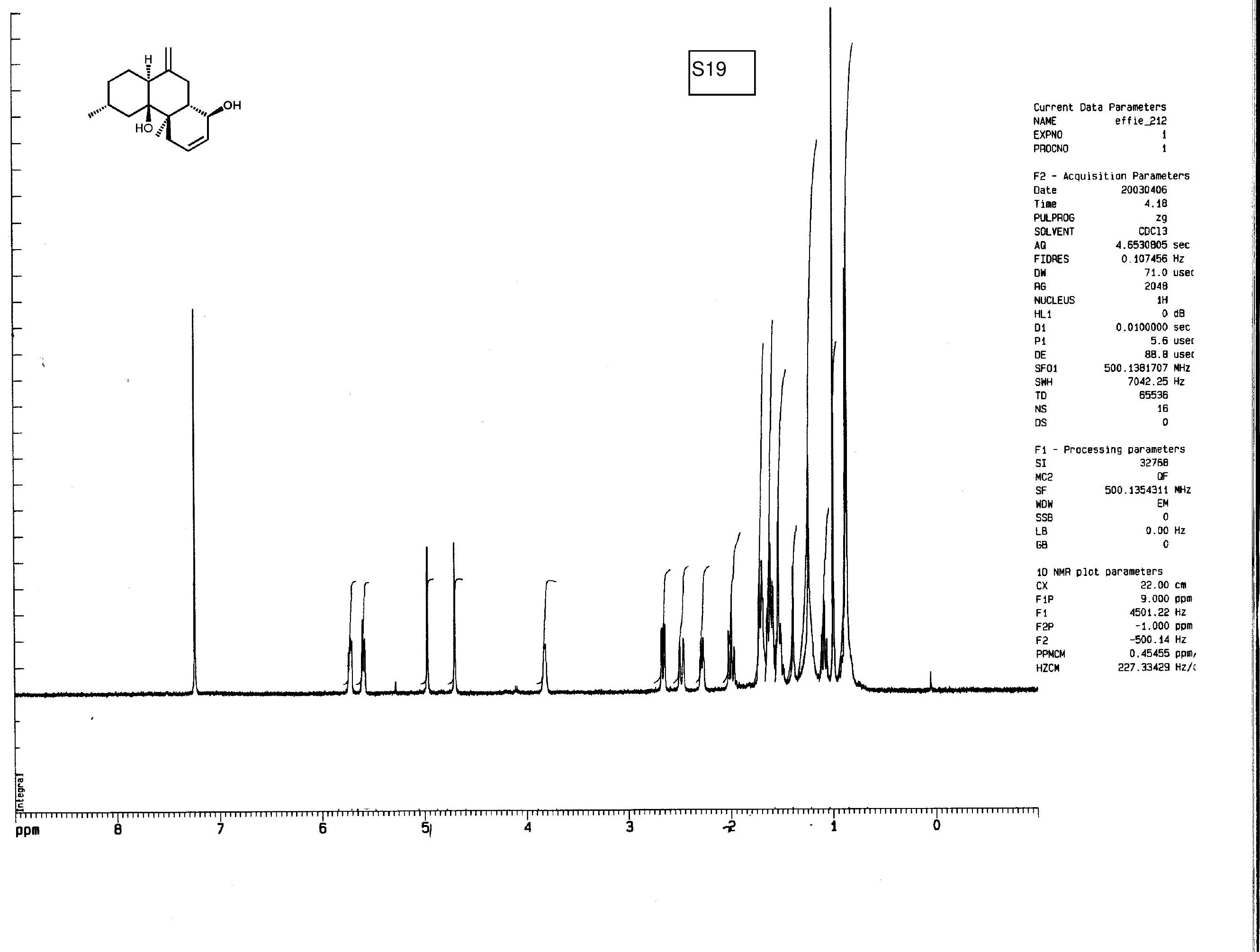




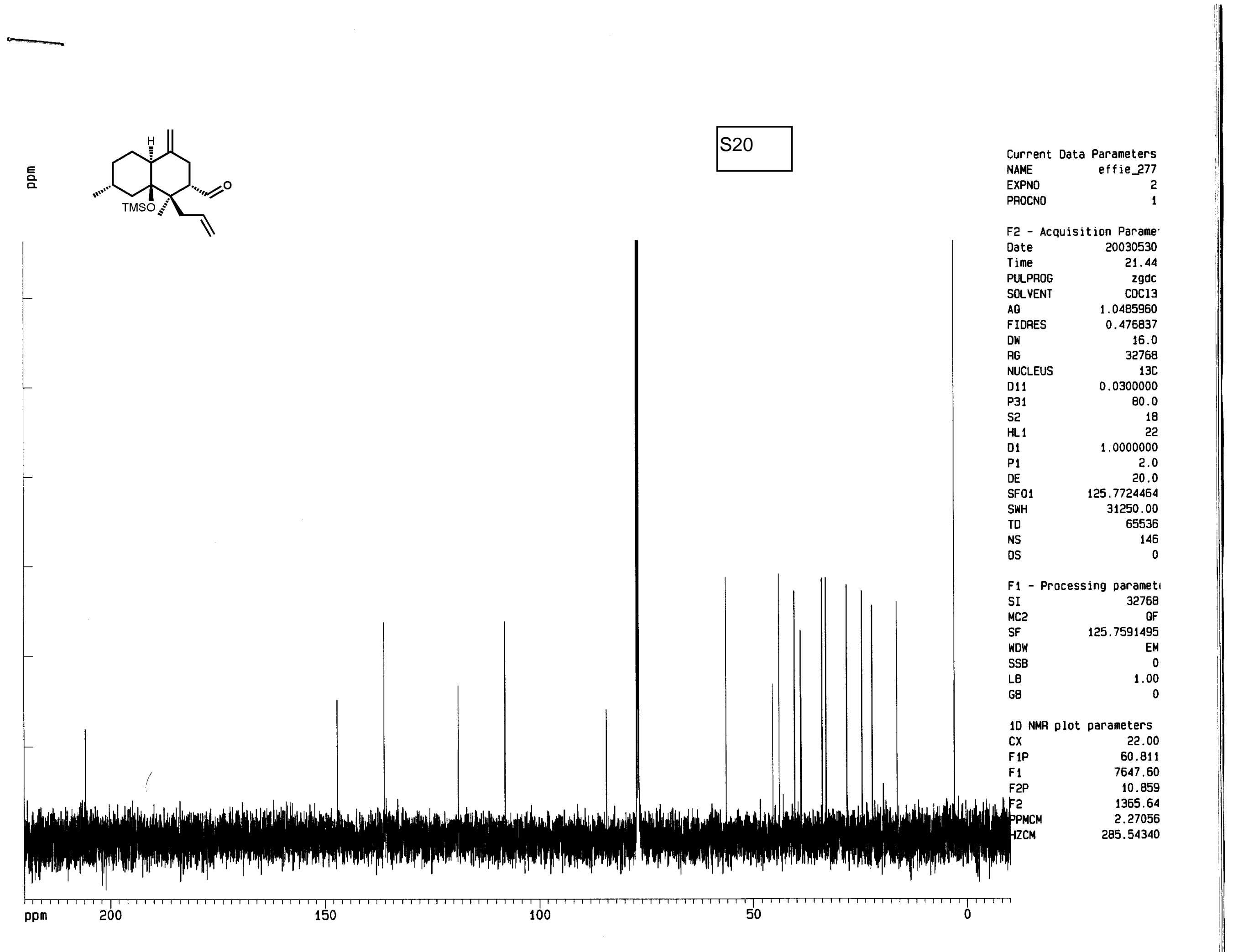




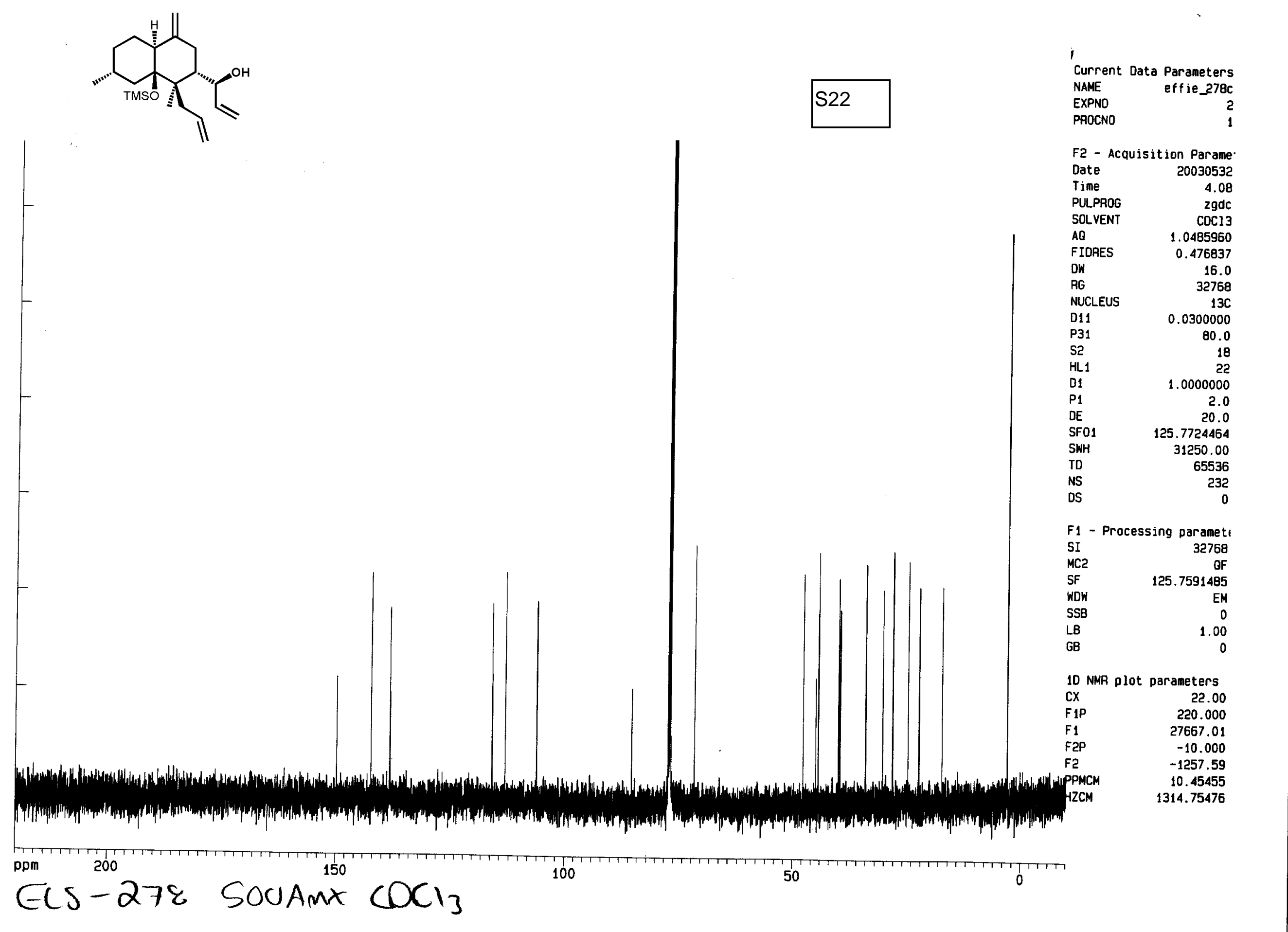




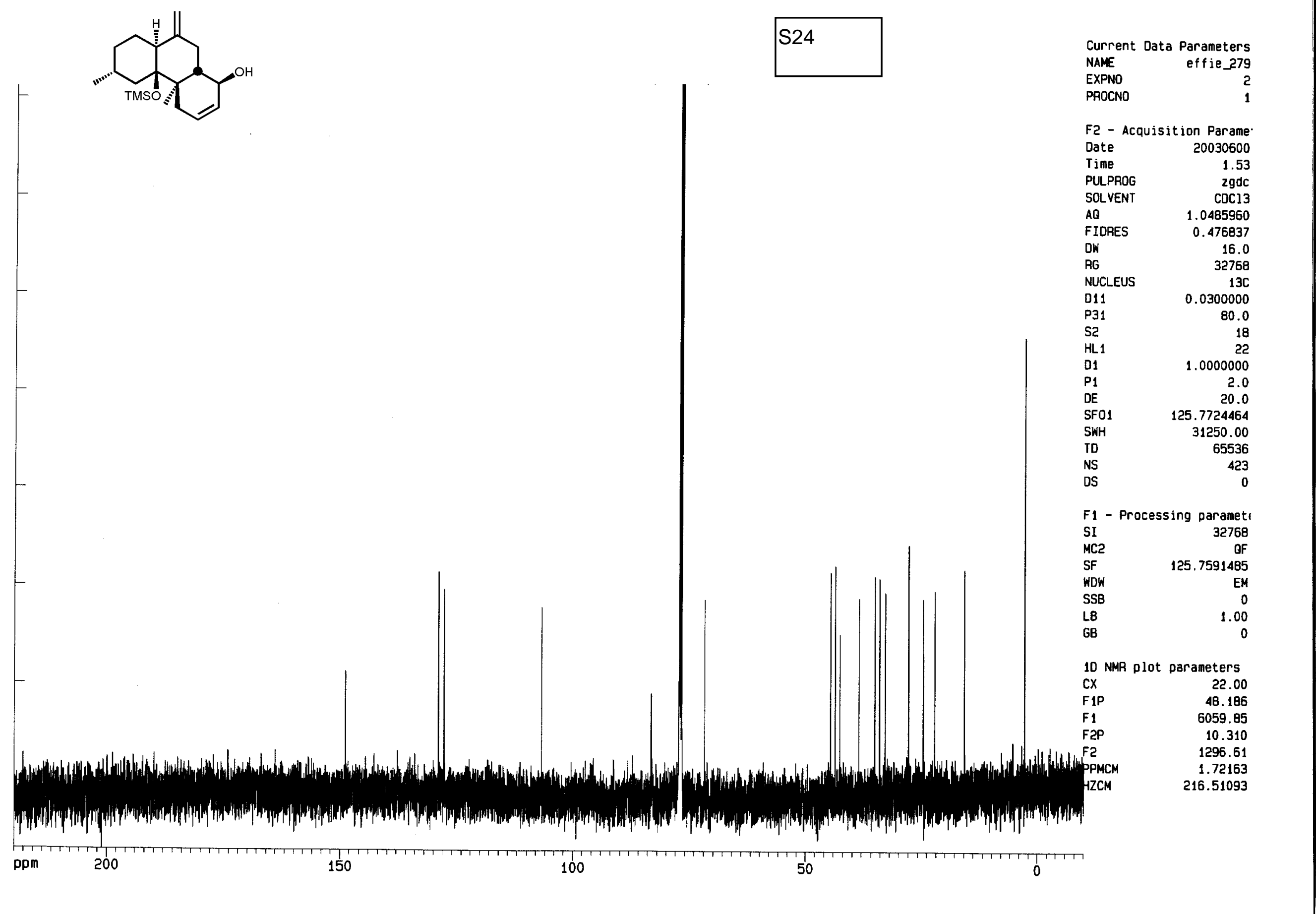




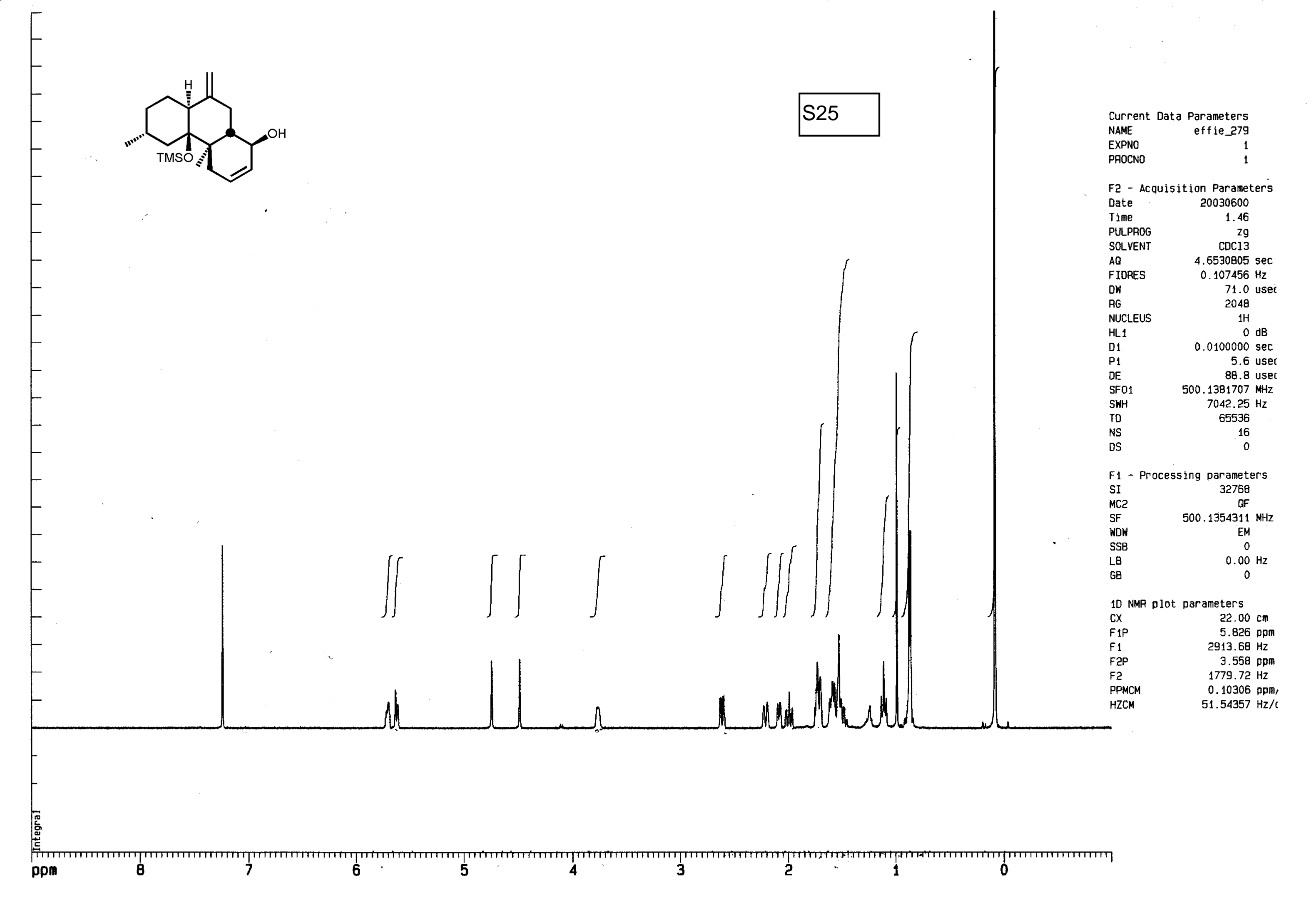


F2 - Acquisition Parameter

Date_ 20030610

$\begin{array}{lr}\text { Time } & 16.18 \\ \text { INSTAUM av } 300\end{array}$

PROBHD $5 \mathrm{~mm}$ QNP $1 \mathrm{H} / 1$

PULPAOG 29pg30

$\begin{array}{ll}\text { TO } & 32768 \\ \text { SOLVENT } & \text { CDC13 }\end{array}$

NS

DS

$\begin{array}{lr}\text { SWH } & 0 \\ & 17985.611 \mathrm{~Hz}\end{array}$

FIDAES $\quad 0.548877 \mathrm{~Hz}$

AQ $\quad 0.9110004 \mathrm{sec}$

$\begin{array}{ll}\text { RG } & 8192 \\ \text { OW } & 27.800 \text { usec }\end{array}$

$\begin{array}{lr}\text { DW } & 27.800 \text { usec } \\ \text { DE } & 6.00 \text { usec }\end{array}$

TE $\quad 300.0 \mathrm{~K}$

D1 $\quad 1.00000000 \mathrm{sec}$

d11 $\quad 0.03000000 \mathrm{sec}$

$==== \pm== \pm x==$ CHANNEL $f 1$

$\begin{array}{ll}== \pm== \pm= \pm== & \text { CHANNEL } f 1== \pm= \\ \text { NUC1 } & 13 \mathrm{C} \\ \text { P1 } & 5.00 \text { usec } \\ \text { PL1 } & -6.00 \mathrm{dg}\end{array}$

$\begin{array}{lr}\text { PL1 } & -6.00 \mathrm{~dB} \\ \text { SF01 } & 75.4752653 \mathrm{MHz}\end{array}$

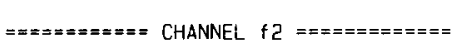

CPDPPG2 waltz16

NUC2 $\quad 1 \mathrm{H}$

PCPO2 $\quad 70.00$ usec

PL2 $\quad-3.00 \mathrm{~dB}$

PL12 $13.48 \mathrm{~dB}$

PL13 $\quad 13.63$ dB

$4860 \mathrm{MHz}$

F2 - Processing parameters

SI $\quad 65536$

SF $\quad 75.4677511$ MH

WOW

SSB
LB
GB
PC

1.40

10 NMP plot parameters

CX $\quad 20.00 \mathrm{~cm}$

$\mathrm{Cr} \quad 12.50 \mathrm{~cm}$

$\begin{array}{lr}\text { F1P } & 220.000 \mathrm{pom} \\ \mathrm{F} 1 & 16602.90 \mathrm{~Hz}\end{array}$

$\begin{array}{ll}\text { F2P } & -10.000 \mathrm{ppm} \\ \text { F2 } & -754.68 \mathrm{~Hz}\end{array}$

$\begin{array}{ll}\text { F2 } & -754.68 \mathrm{~Hz} \\ \text { PPMCM } & 11.50000 \mathrm{pom} / \mathrm{Cm}\end{array}$ 

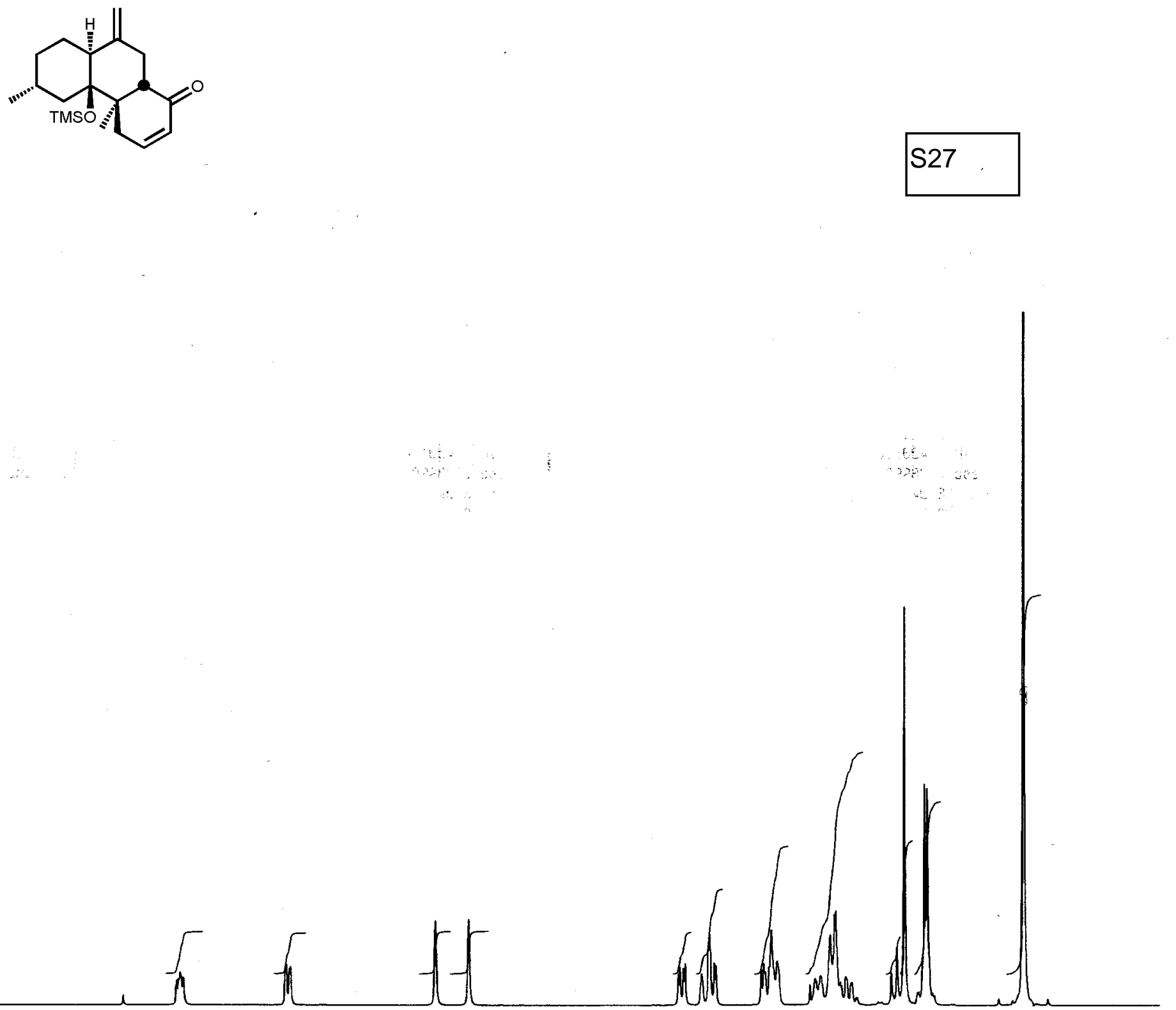

Current Data Parameters

NAME effie_289

EXPNO

PROCNO

F2 - Acquisition Parameters

Date_ 20030610

16.15
av 300

INSTRUM av300

PAOBHD $5 \mathrm{~mm}$ QNP $1 \mathrm{H} / 1$

PULPROG $\quad 2930$

TD $\quad 30720$

NS CDCl3

DS

SWH

$\quad 5081.301 \mathrm{~Hz}$

$A Q$

3.0228980. sec

$\begin{array}{lc}\text { AG } & 45.3 \\ \text { OW } & 98.400 \text { usec }\end{array}$

$\begin{array}{lr}\text { DE } & 98.400 \text { usec } \\ \text { DE } & 6.00 \text { usec }\end{array}$

$300.0 \mathrm{~K}$

$1.00000000 \mathrm{sec}$

$== \pm=== \pm=x=\pi=$ CHANNEL $f 1$

$\begin{array}{lc}\text { NUC1 } & 1 \mathrm{H} \\ \mathrm{P} 1 & 10.50 \text { use }\end{array}$

$\begin{array}{ll}\text { P1 } & 10.50 \text { US } \\ \text { PL1 } & -3.00 \mathrm{~dB}\end{array}$

SF01 300.1319477 MHZ

F2 - Processing parameters

SI 65536

SF $\quad 300.1300000 \mathrm{MHz}$

WOW EM

SSB $\quad 0$

$\begin{array}{lr}\text { GB } & 0.10 \\ \text { PC } & 0\end{array}$

10 NMP plot parameters

$\mathrm{CX} \quad 20.00 \mathrm{cI}$

CY $\quad 20.00 \mathrm{~cm}$

F1P $\quad 9.000 \mathrm{ppm}$

F1 $2701.17 \mathrm{~Hz}$

F2P $\quad-1.000 \mathrm{ppm}$

F2 $-300.13 \mathrm{~Hz}$

$\begin{array}{lr}\text { PPMCM } & 0.50000 \mathrm{ppm} / \mathrm{cm} \\ \text { HZCM } & 150.06500 \mathrm{~Hz} / \mathrm{cm}\end{array}$ 
Current Data Parameters NAME effie_290

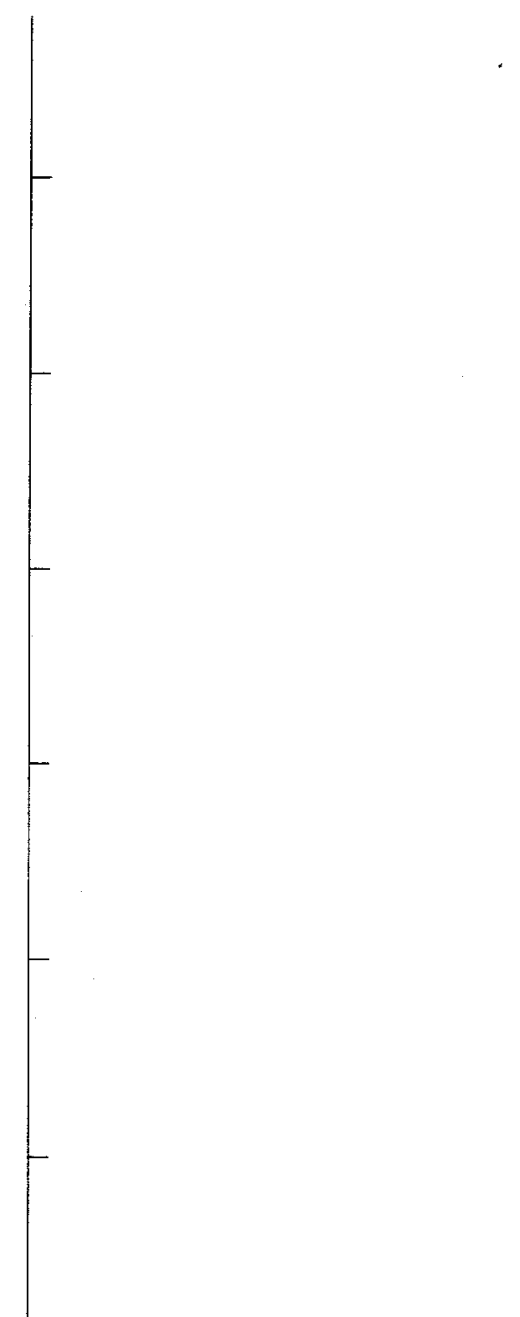

F2 - Acquisition Parame

$\begin{array}{lr}\text { Date } & 20030612 \\ \text { Time } & 11.44\end{array}$

Time

Time

SOLVEN

AQ

2gdc

$\mathrm{COCl3}$

$\quad 1.0485960$

DW

AG

NUSLEUS

011

S2

HL1

01

P1

SF01

SWH

SWH

TD

NS

0.476837

16.0

32768 0.0300000

18

1.0000000

2.0
20.0

125.7724464

31250.00

65536

F1 - Processing parameti

SI

MC2

QF
125.7591514

SF $\quad 125.7591514$

WDW

LB

LB

10 NMA plot parameters

CX 22.00

F1P $\quad 220.000$

F1 27667.01

$\begin{array}{lr}F 1 & 27667.01 \\ F 2 P & -10.000\end{array}$

F2 -1257.59

PPMCM 10.45455

1314.75476 


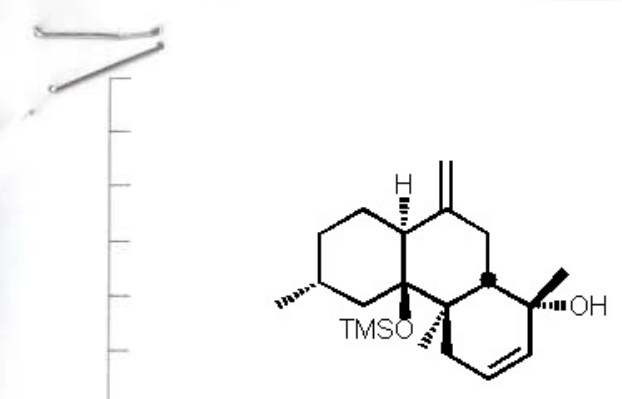

Current Data Parameters

NAME effie 290

1

S29

F2 - Acquisition Parameters

Date 20030612

Time

PULPAOC

SOLVENT

AQ
FIDRES

11.38
79

2.053005

4. $6530805 \mathrm{sec}$

$0.107456 \mathrm{~Hz}$

PG

NUCLEUS

HL:

$D 1$
$P 1$

DE

SWH

TW

NS

F1 - Processing parameters

MC2
SF

SF $\frac{\text { GF }}{500.1354311 \mathrm{MHz}}$

SSP
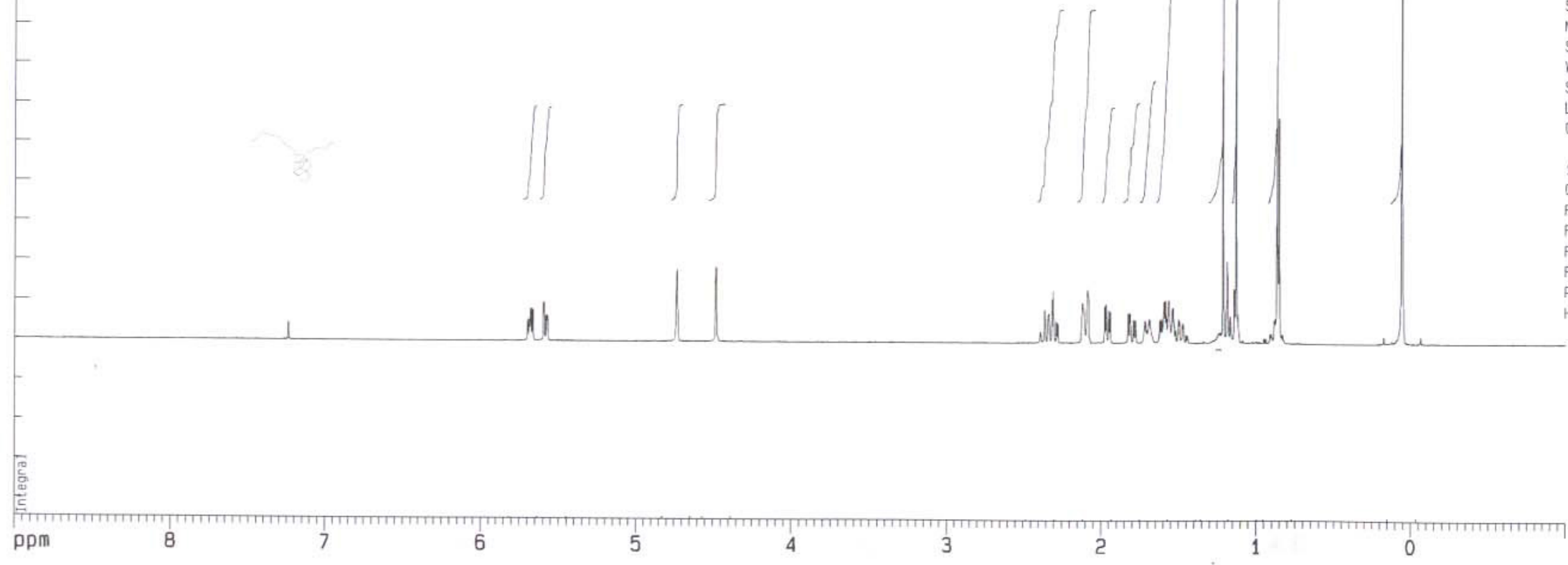
PROCNO

F2 - Acquisition Parameter

Date_ 20030611

$\begin{array}{ll}\text { Time } & 17.41 \\ \text { INSTRUM } & \text { ay300 }\end{array}$

PROBHD 5 m ONP 1 av300

PULPROG 5 Th 20530

\begin{tabular}{lr} 
TD & 290930 \\
TD & 32768 \\
\hline
\end{tabular}

SOLVET

NS

$\begin{array}{lr}\text { DS } & 0 \\ \text { SWH } & 17985.611 \mathrm{~Hz} \\ \text { FIDRES } & 0.548877 \mathrm{~Hz}\end{array}$

FIDRES $\quad 0.548877 \mathrm{~Hz}$

AG $\quad 0.9110004 \mathrm{sec}$

$\begin{array}{ll} & 3649.1 \\ \text { DW } & 27.800\end{array}$

DE $\quad 6.00$ usec

TE $\quad 300.0 \mathrm{~K}$

$01 \quad 1.00000000 \mathrm{sec}$

d11 $0.03000000 \mathrm{sec}$

$==========$ CHANNEL $f 1$

$\begin{array}{lr}\text { NUC1 } & 13 \mathrm{C} \\ \text { P1 } & 5.00 \text { use } \\ \text { PL1 } & -6.00 \mathrm{~dB}\end{array}$

SF01 $75.4752653 \mathrm{MHZ}$

$======s= \pm==$ CHANNEL $f 2$

NUC2 Waltz16

PCPO2 $\quad 70.00$ usec

PL2 $\quad-3.00 \mathrm{~dB}$

PL12 $13.48 \mathrm{~dB}$

$\begin{array}{lr}\text { PL13 } & 15.63 \mathrm{~dB} \\ \text { SF02 } & 300.1314860 \mathrm{MH} 2\end{array}$

F2 - Processing parameters

SI $\quad 65536$

SF $\quad 75.4677503 \mathrm{MHZ}$

WOW

$\begin{array}{lc}\text { SSB } & 0 \\ \mathrm{LB} & 1.00 \mathrm{~Hz} \\ \mathrm{~GB} & 0\end{array}$

$$
\begin{array}{r}
0 \\
1.40
\end{array}
$$

10 NMR plot parameters

$\begin{array}{ll}\text { CX } & 20.00 \mathrm{~cm} \\ \mathrm{CY} & 12.50 \mathrm{~cm}\end{array}$

F1p $\quad 220.000 \mathrm{ppm}$

F1 $\quad 16602.91 \mathrm{~Hz}$

F2P $\quad-10.000 \mathrm{pp}$

$\begin{array}{lll}-10 & -754.68 \mathrm{~Hz}\end{array}$

$\begin{array}{lr}\text { TPMCM } & 11.50000 \mathrm{ppm} / \mathrm{cm} \\ \text { HZCM } & 867.87921 \mathrm{~Hz} / \mathrm{cm}\end{array}$

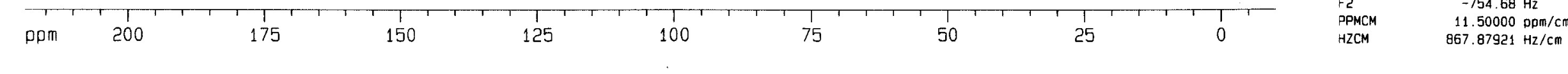




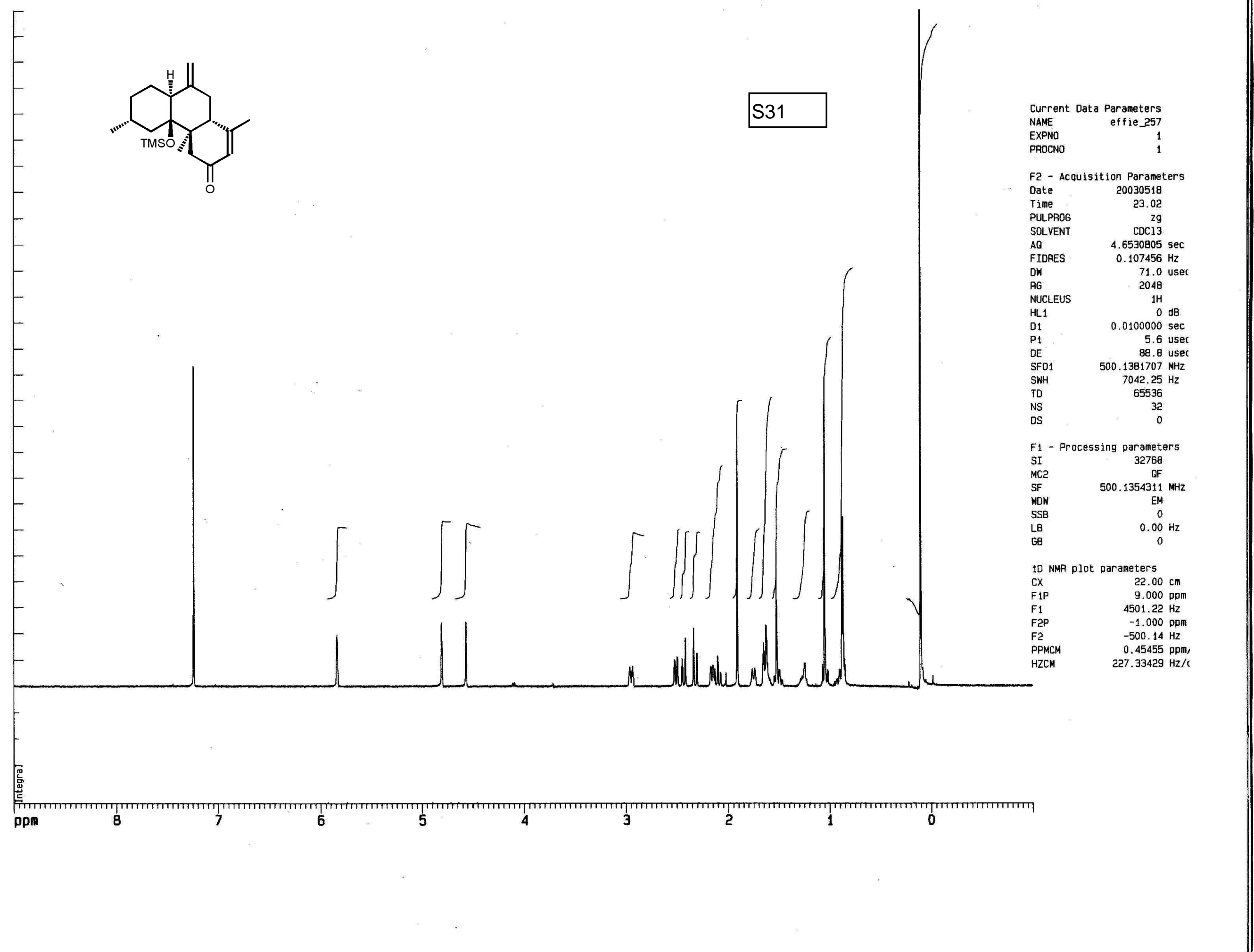



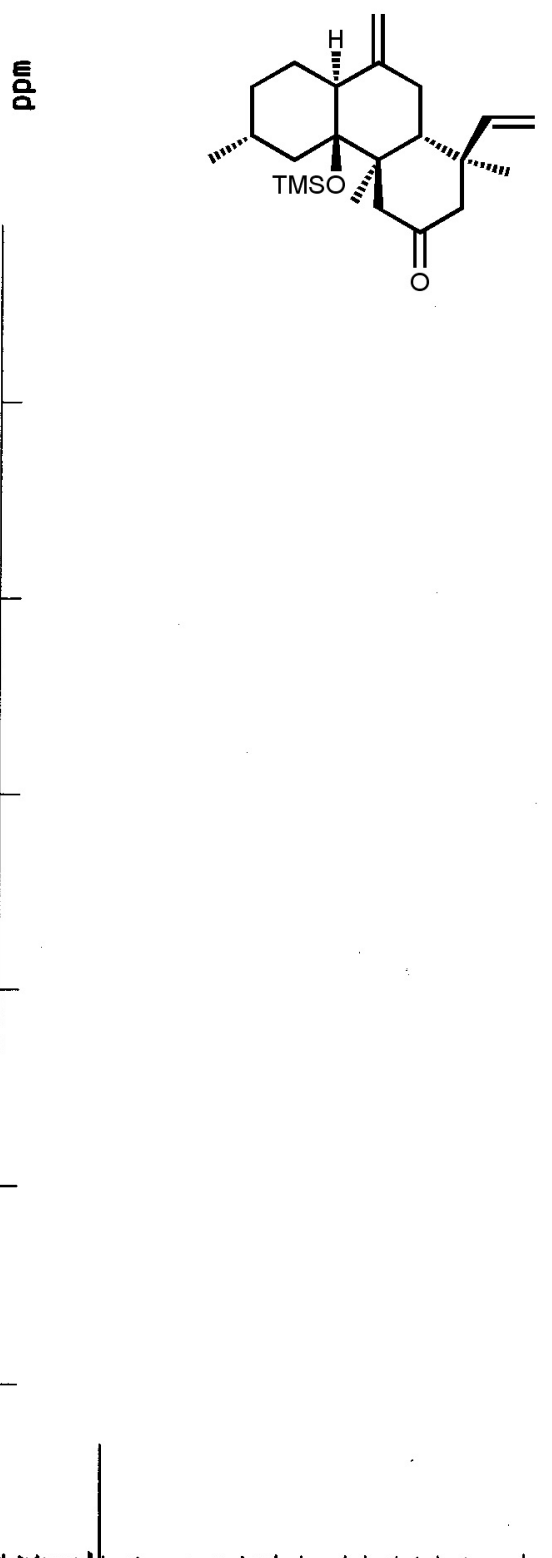

Current Data Parameters

F2 - Acquisition Parame

Date 20030626

Time $\quad 14.49$

PULPROG 2gdc

SOLVENT

$A B$

FIDAES

DW

AG

NUCLEUS

011

S2

HL1

01

PI

SF01

SWH

TD

1.0485960

0.476837

16.0
32768

0.0300000

80.0
18

22
1.0000000

$$
\begin{array}{r}
2.0 \\
20.0
\end{array}
$$

125.7724464

31250.00

31250.00

65536
1536

F1 - Processing paramet

SI 32768

MC2 OF

$\begin{array}{lr}\text { MC2 } & \text { of } \\ \text { SF } & 125.7591187\end{array}$

$\begin{array}{lr}\text { WDW } & 125.7591187 \\ & \text { EM }\end{array}$

SSB

LB

GB

10 NMP plot parameters

$\begin{array}{lr}\text { CX } & 22.00 \\ \text { F1P } & 59.165\end{array}$

$\begin{array}{lr}F 1 P & 59.165 \\ F 1 & 7440.50\end{array}$

F1 $\quad 7440.50$

F2P $\quad 14.153$

1779.84

HZCM 2.04600

257.30276 


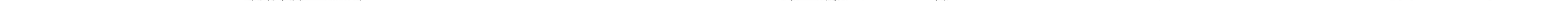




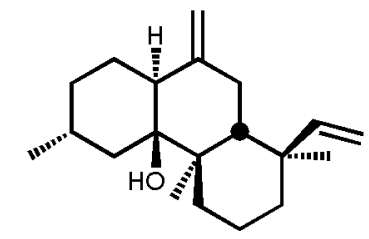

Current Data Parameters

EXPNO

PAOCNO

F2 - Acquisition Parame

Date 20030716

9.18

SOLVENT COC13

$\begin{array}{lr}\text { AO } & \text { COC13 } \\ \text { POLVENT } & 1.0485960\end{array}$

FIDRES $\quad 0.476837$

$\begin{array}{lr}\text { OW } & 16.0 \\ \text { AG } & 32768\end{array}$

NUCLEUS $\quad 13 \mathrm{C}$

$011 \quad 0.0300000$

P31 80.0

$\begin{array}{ll}S 2 & 18 \\ H L 1 & 22\end{array}$

$\begin{array}{ll}H L 1 & 1.0000000 \\ 01 & \end{array}$

$\begin{array}{lr}\text { P1 } & 2.0 \\ D E & 20.0\end{array}$

SF01 125.7724464

SWH 31250.00

TD 65536

NS 1030

F1 - Processing parametı

SI 32768

MC2 $\quad$ OF

SF $\quad 125.7591187$

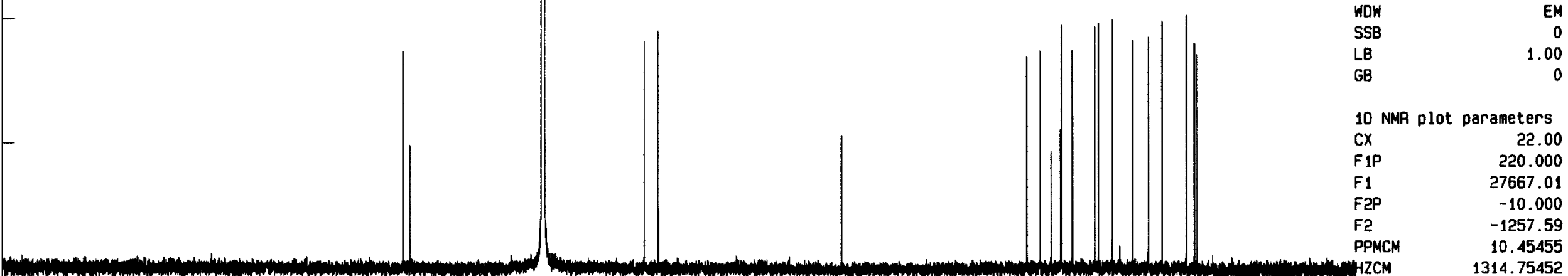



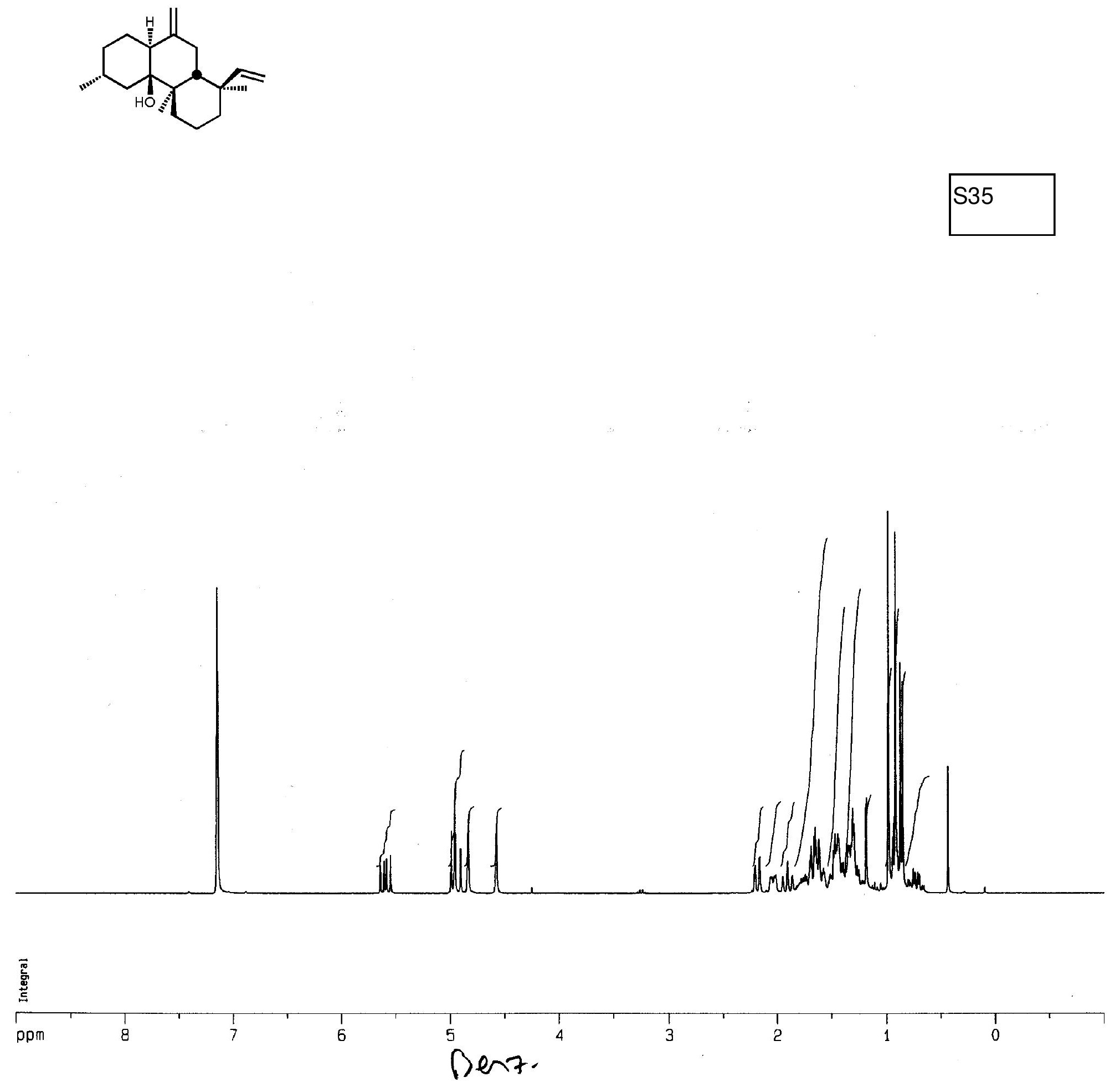

Current Data Parameters NAME effie $338 \quad 340$

F2 - Acquisition Parameters Date_ 20030717

Time

INSTRUM $\quad 14.05$

PAOBHD $5 \mathrm{~mm}$ ONP $1 \mathrm{H} / 1$

PULPROG

TD 30720

SOLVENT

NS $\quad 16$

DS

SWH $5081.301 \mathrm{~Hz}$

IDRES $\quad 0.165407 \mathrm{~Hz}$

AQ $\quad 3.0228980 \mathrm{sec}$

AG 228.1

$\begin{array}{lr}\text { DW } & 98.400 \text { usec } \\ \text { DE } & 6.00 \text { usec }\end{array}$

TE $300.0 \mathrm{~K}$

$01 \quad 1.00000000 \mathrm{sec}$

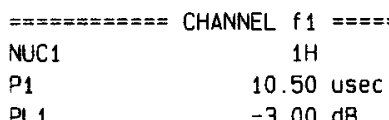

PL1 -3.00 dB

SF01 300.1319477 MHZ

F2 - Processing parameters

SI 65536

SF $\quad 300.1300000 \mathrm{MHz}$

WDW 300.130000

SSB 0.

$\begin{array}{lr}\text { LB } & 0.10 \mathrm{H} \\ \text { GB } & 0\end{array}$

$\begin{array}{ll}P C & 1.00\end{array}$

10 NMR plot parameters

CX $\quad 20.00 \mathrm{~cm}$

$\mathrm{Cr} \quad 7.00 \mathrm{~cm}$

F1P $\quad 9.000 \mathrm{ppm}$

F1 $2701.17 \mathrm{~Hz}$

F2P $\quad-1.000 \mathrm{pom}$

$-300.13 \mathrm{~Hz}$

$\quad 0.50000 \mathrm{ppm} / \mathrm{cm}$

$\mathrm{HZCM} \quad 150.06500 \mathrm{~Hz} / \mathrm{cm}$ 


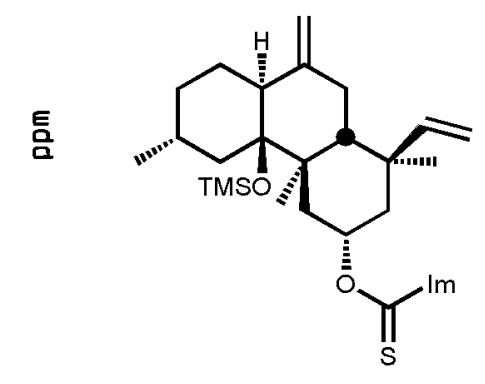

Current Data Parameters

MAME

EXPNO

effie_447

PROCNO

F2 - Acquisition Parame

Date 20031020

Time $\quad 3.55$

PULPROG 2gdc

SOLVENT COC13

AQ $\quad 1.0485960$

$\begin{array}{lr}\text { FIDRES } & 0.476837 \\ \text { DW } & 16.0\end{array}$

$\begin{array}{rr}\text { DW } & 16.0 \\ \text { RG } & 32768\end{array}$

NUCLEUS

0.0300000

P31

HL1

DI 1

P1

$\mathrm{DE}$

SFO

SWH

TD

NS

18

00000

2.0
20.0

125.7724464

31250.00

3536

3838

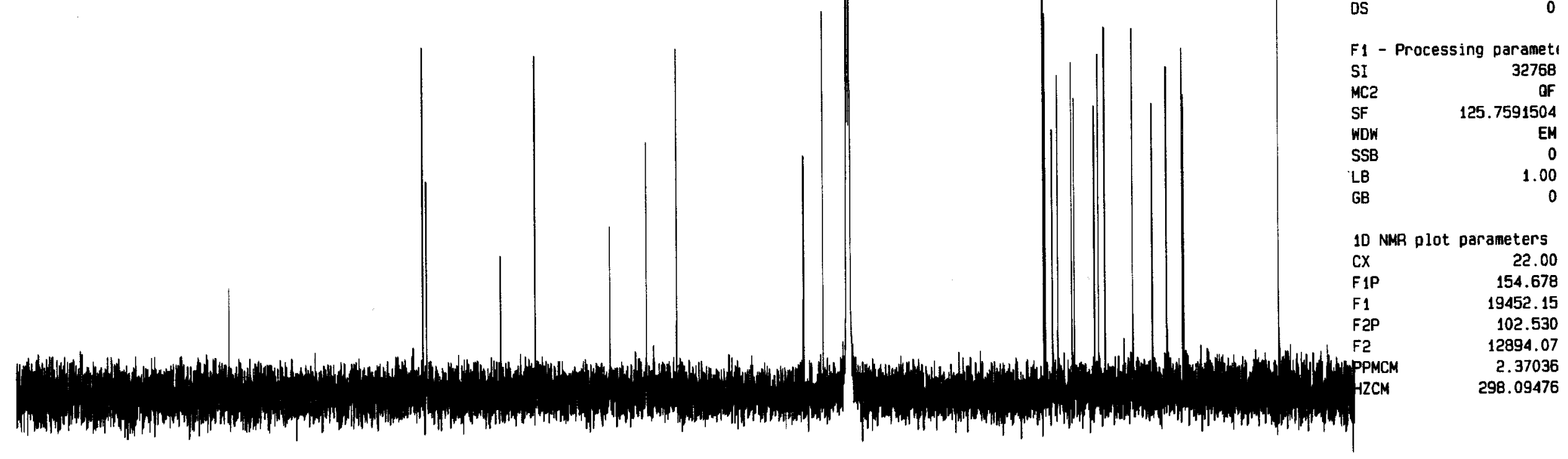



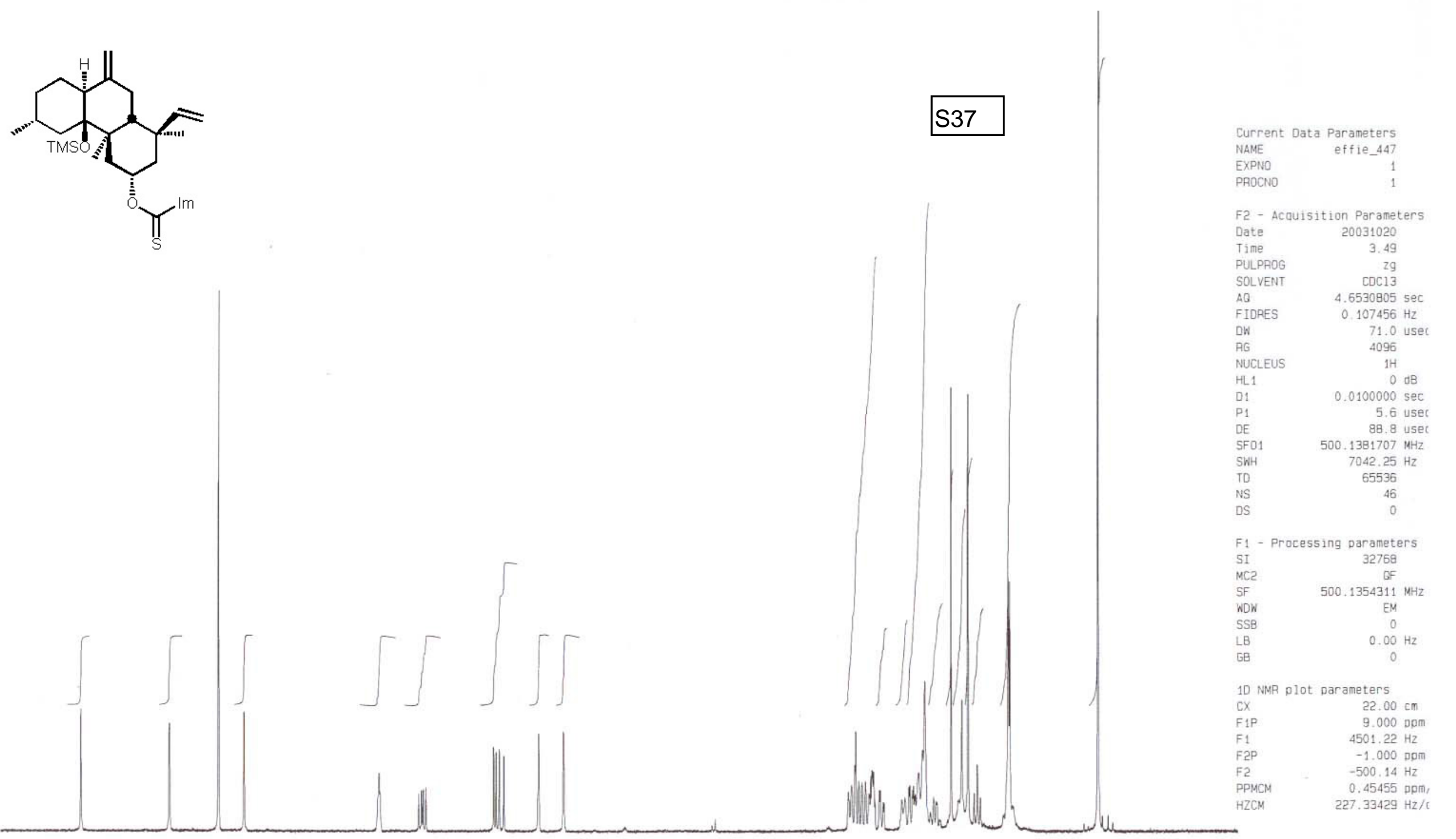


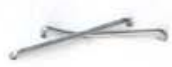

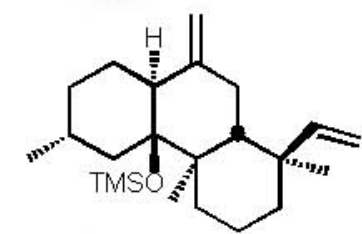

言

S38
Current Data Parameters

NAME effie 450

effie_450

F2 - Acquisition Parame

Date 2003102

20031020

PULPROG $\quad \begin{array}{ll}1.23 \\ \text { 2gdc }\end{array}$

SOLVENT COC13

AQ

1.0485960

FIDRES $\quad 0.476837$

DW $\quad 16.0$

RU $\quad 32768$

0.0300000

$\begin{array}{lr}\text { P31 } & 0.0300000 \\ & 80.0\end{array}$

S2 18

$\begin{array}{lr}\text { HL1 } & 1.0000000 \\ 01 & \end{array}$

P1 2.0

DE 20.0

125.7724464

SWH $\quad 31250.00$

TD 65536

$\begin{array}{lr}\text { NS } & 244 \\ \text { DS } & 0\end{array}$

F1 - Processing parameti

SI 32768

WDW

SSB

LB

125.7591495

125.7591495

0

1.00
0

10 NMR plot parameters

CX 22.00

Fip

F1

Fip

$\mathrm{F} 2$

PPMCM

IZCM

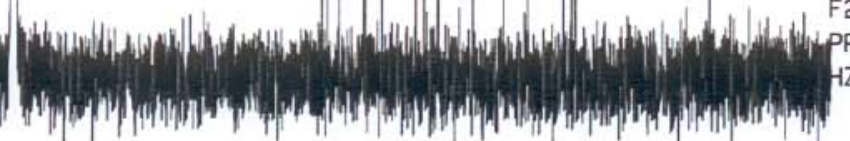

155.227

19521.18

81.671

10270.83

40.47052

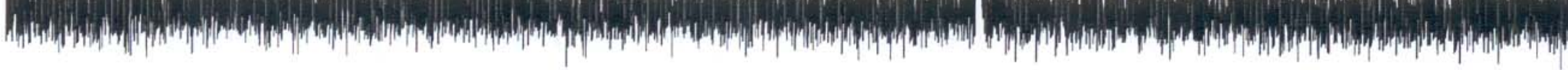



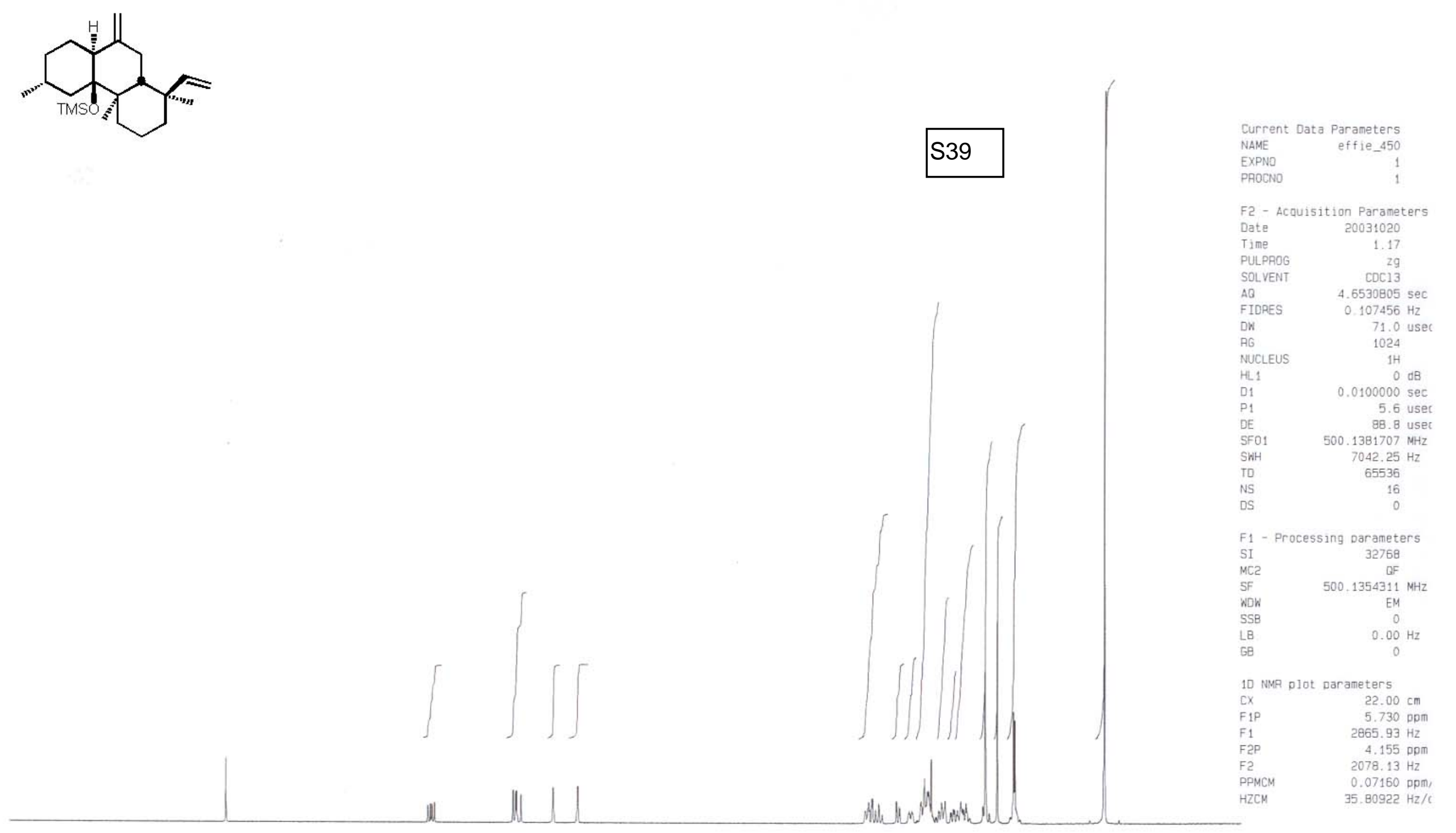


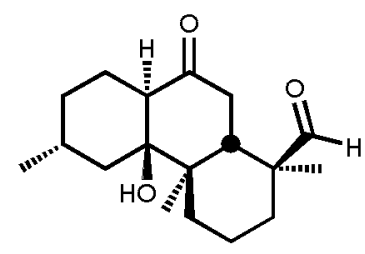

Current Data Parameters

NAME effie_440Pd

NAME effie_440Pd

S40 PAOCNO

F2 - Acquisition Parame.

Date 20031012

$\begin{array}{rr}\text { Date } & \text { Time } \\ \text { PULPROG }\end{array}$

PULPROG 2gdc

SOLVENT $\quad \mathrm{COC13}$

$\begin{array}{lr}A Q & 1.0485960 \\ \text { FIDRES } & 0.476837\end{array}$

16.0

DG 32768

NUCLEUS $\quad 13 \mathrm{C}$

D11 $\quad 0.0300000$

P31 80.0

$\begin{array}{ll}\text { S2 } & 18 \\ \text { HL1 } & 22 \\ \text { D1 }\end{array}$

$01 \quad 1.0000000$

P1 2.0

SFO1 $\quad 125.7724464$

$\begin{array}{lr}\text { SFO1 } & 125.7724464 \\ \text { SWH } & 31250.00\end{array}$

$\begin{array}{lr}\text { SWH } & 31250.00 \\ \text { TO } & 65536\end{array}$

$\begin{array}{lr}\text { NS } & 1386 \\ \text { DS } & 0\end{array}$

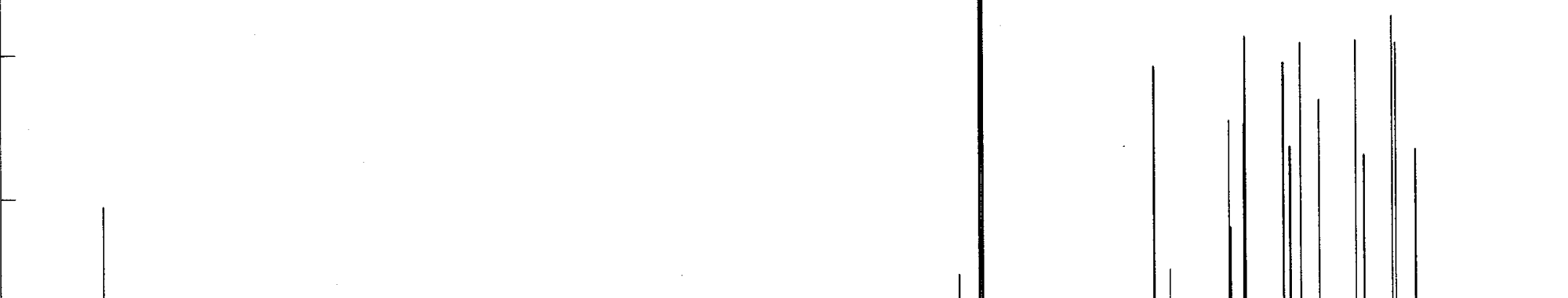

1 - Processing paramet SI 32768 $\begin{array}{lr}\text { SI } & 32768 \\ \text { MC2 } & \text { OF }\end{array}$ $\begin{array}{lr}\text { MC2 } & \text { OF } \\ \text { SF } & 125.7591495\end{array}$ $\begin{array}{lr}\text { WDW } & \text { EM } \\ \text { SSB } & 0\end{array}$

10 NMR plot parameters CX 22.00 FIP $\quad 56.969$ F1 7164.37 F2P $\quad 7.566$ F2 $\quad 951.45$ PPMCM 2.24561 282.40555 Wr 


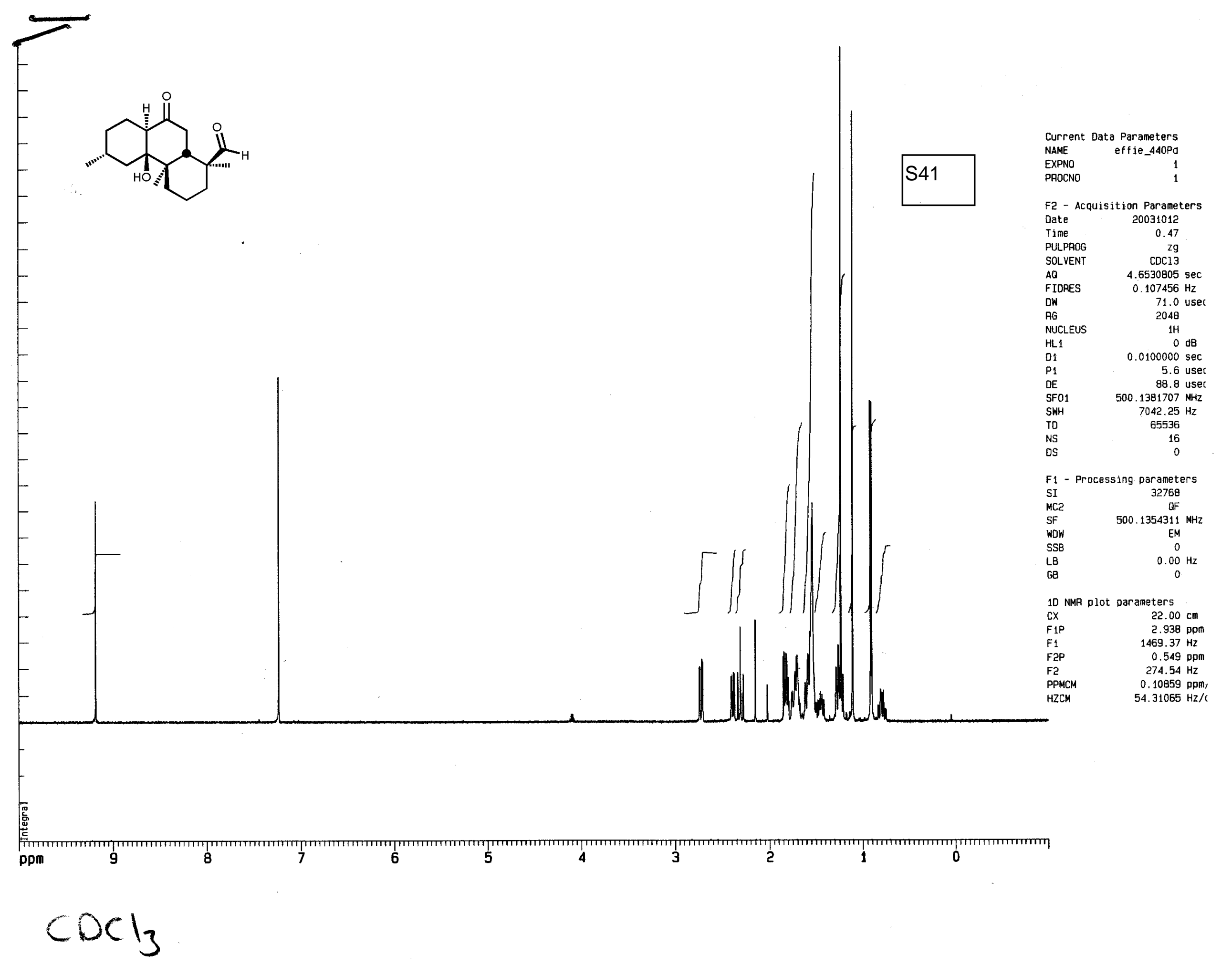



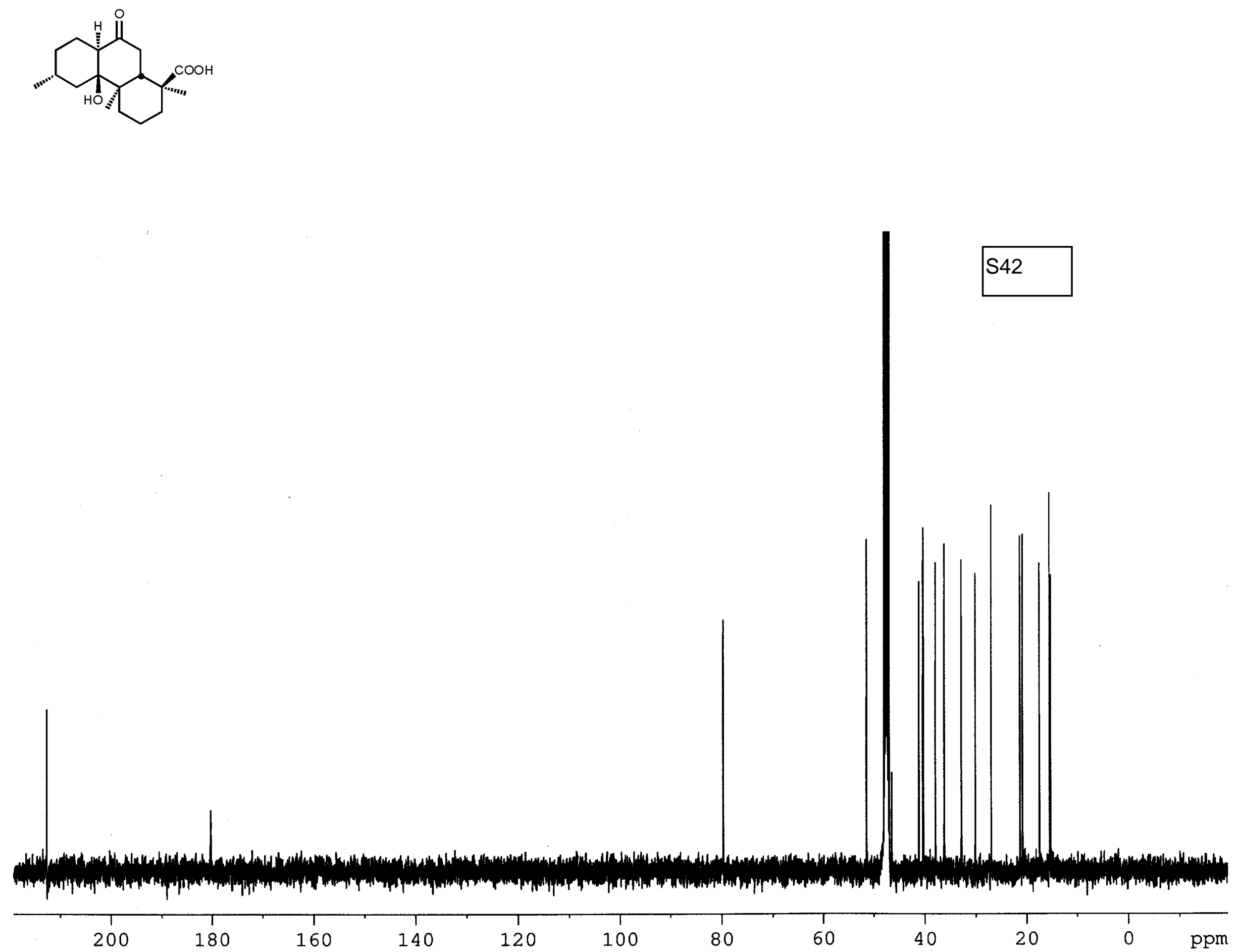
$13 \mathrm{C}$ with proton decoupling
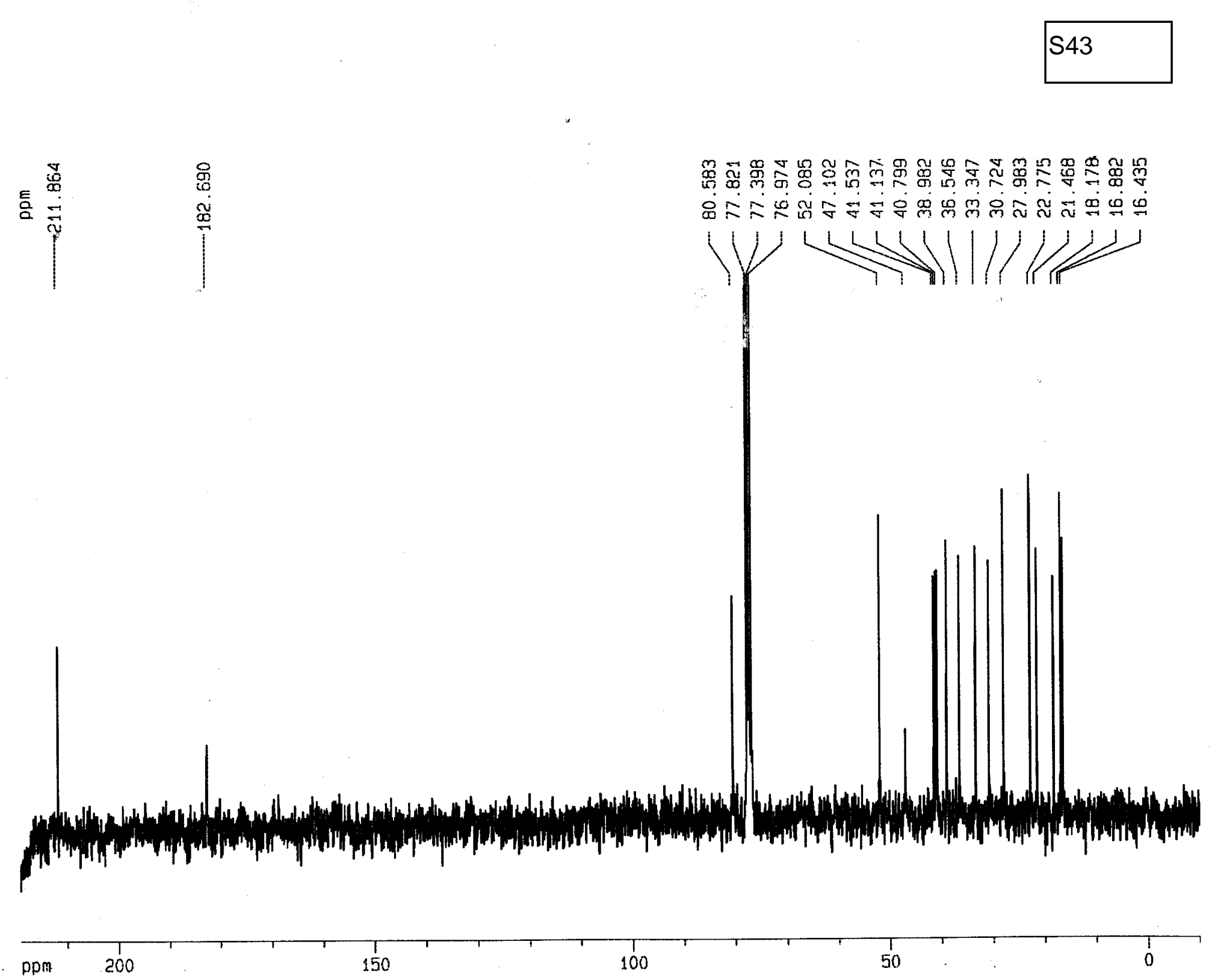

Current Data Parameters

NAME effie_J05acid_cacla

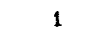

F2 - Acquisition Parameters

Date 20040623

Time 17.50

PROBH 5 m ONP $1 \mathrm{H} / 1$

PULPROS 5 in 2014

29pg30

SOLVENT COC13

$\begin{array}{lr}\text { NS } & 275 \\ \text { DS } & 0\end{array}$

SWH $\quad \begin{aligned} & 17985.611 \mathrm{~Hz} \\ & \text { FIDRES } 0.548877 \mathrm{~Hz}\end{aligned}$

FIDRES $\quad 0.548877 \mathrm{~Hz}$

AO $\quad 0.9110004$ sec

$\begin{array}{ll}\text { AG } & 3649.1 \\ \text { DW } & 27.800 \text { usec }\end{array}$

DE $\quad 6.00$ usec

TE

$01 \quad 1.00000000 \mathrm{sec}$

di1 $\quad 0.0300000 \mathrm{sec}$

0.00002000 sec

$== \pm= \pm== \pm==$ CHANNEL $f$

$\begin{array}{ll}\text { NuC1 } & 13 \mathrm{C} \\ \mathrm{Pl} & 5.00 \mathrm{usec}\end{array}$

PL1 $\quad-6.00 \mathrm{~dB}$

SFO1 $\quad 75.4752653 \mathrm{MHZ}$

$========= \pm$ CHANNEL $f 2=$
waltz16

$\begin{array}{ll}\text { PCPO2 } & 70.00 \text { usec } \\ \text { Pl2 } & -3.00 \mathrm{~dB}\end{array}$

$\begin{array}{ll}\text { PL2 } & -3.00 \mathrm{~dB}\end{array}$

PL13 $15.63 \mathrm{~dB}$

SF02 $300.1314860 \mathrm{MHZ}$

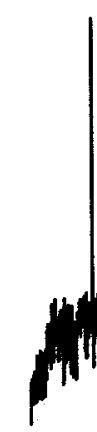

72 - Processing parameters

SI 65536

SF $\quad 75.4677190 \mathrm{MHz}$

WOW $\quad$ SSB

$\begin{array}{lc}\angle B & 3.00 \\ \text { GB } & 0 \\ P C & 1.40\end{array}$

10 Nif plot parameters
CX $20.00 \mathrm{c}$

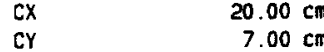

$\begin{array}{ll}\mathrm{CY} & 7.00 \mathrm{~cm} \\ \text { FIP } & 220.000 \mathrm{pom}\end{array}$

F1 $\quad 16602.90 \mathrm{~Hz}$

F2P $\quad-10.000 \mathrm{ppm}$

$F 2 \quad-754.68 \mathrm{~Hz}$

$\begin{array}{lr}\text { PPMCM } & 11.50000 \mathrm{ppm} / \mathrm{Cm} \\ \mathrm{HZCM} & 867.87878 \mathrm{HZ} / \mathrm{cm}\end{array}$ 


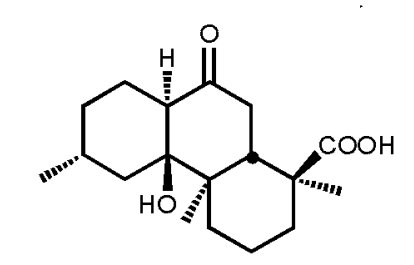

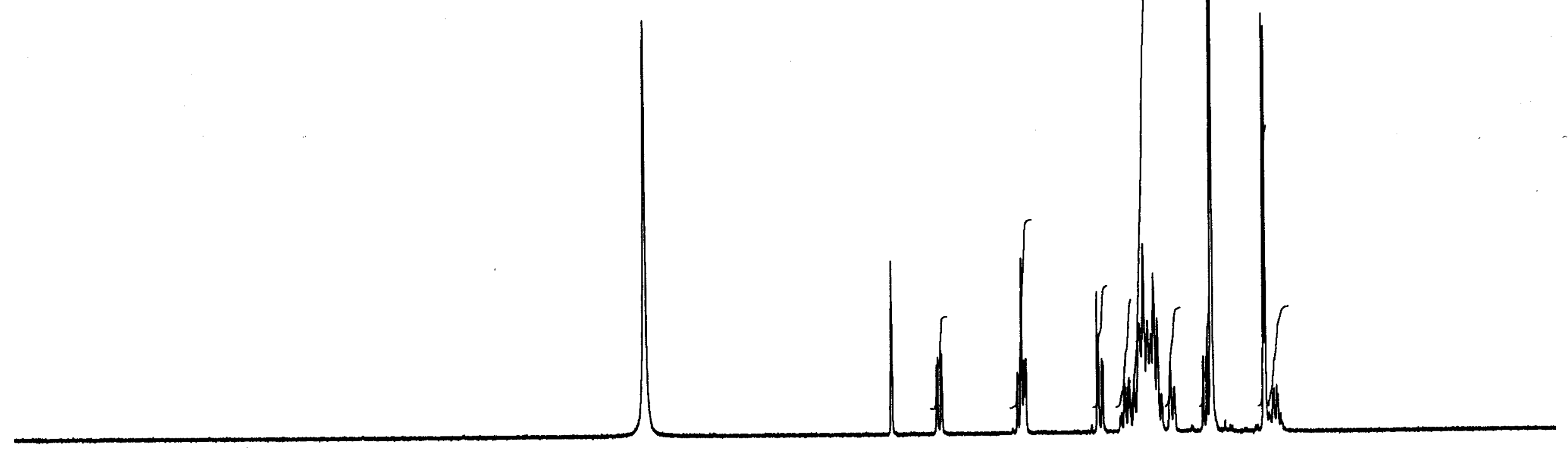




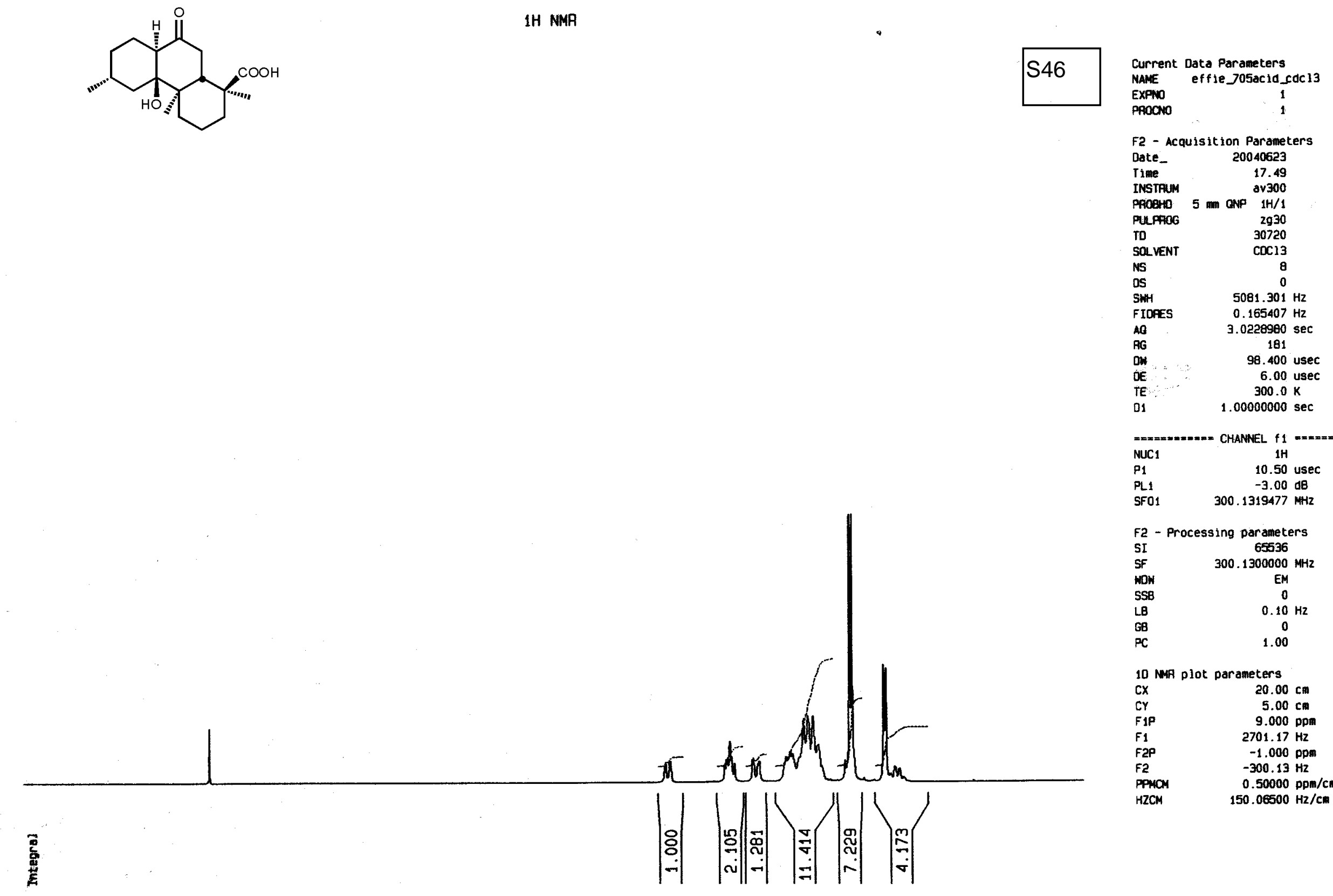

F2 - Acquisition Parameters

Date_ 20040623

Iime $\quad 17.49$

INSTRUM av300

PFOQHO 5 min GNP $1 \mathrm{H} / 1$

$\begin{array}{lr}\text { PULPAOG } & 2930 \\ \text { TD } & 30720\end{array}$

SOLVENT CDC13

NS

os

SWH $\quad 5081.301 \mathrm{~Hz}$

FO $0.165407 \mathrm{~Hz}$

AQ $\quad 3.0228900$

$\begin{array}{lr}\text { AG } & 181 \\ \text { OW } & 98.400 \text { usec }\end{array}$

DE $\quad 6.00$ usec

$01 \quad 1.00000000 \mathrm{sec}$

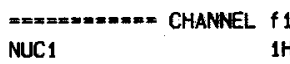

$\begin{array}{lr}\text { NuC1 } & 1 \mathrm{H} \\ \mathrm{P1} & 10.50 \text { use }\end{array}$

PL1 $\quad-3.00 \mathrm{~dB}$

F2 - Processing parameters

$5 I \quad 65536$

SF $\quad 300.1300000 \mathrm{MHz}$

WON

$\begin{array}{lc}\text { SSB } & 0 \\ \text { LB } & 0.10 \mathrm{~Hz}\end{array}$

$\begin{array}{lr}\text { GB } & 0 \\ \text { PC } & 1.00\end{array}$

10 Na plot parameters

$\begin{array}{lr}\mathrm{CX} & 20.00 \mathrm{~cm} \\ \mathrm{Cy} & 5.00 \mathrm{~cm}\end{array}$

FyP $\quad 9.000 \mathrm{ppm}$

F1 $2701.17 \mathrm{~Hz}$

$\begin{array}{ll}F_{22} & -1.000 \mathrm{ppm} \\ F 2 & -300.13 \mathrm{~Hz}\end{array}$

PPMCM $\quad 0.50000 \mathrm{ppm} / \mathrm{cm}$

$\mathrm{HZCM} \quad 150.06500 \mathrm{~Hz} / \mathrm{cm}$ 


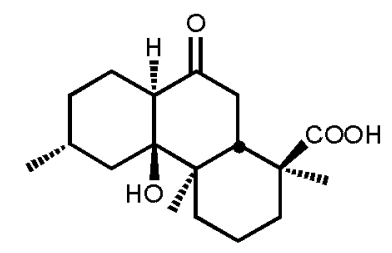

1H NMR

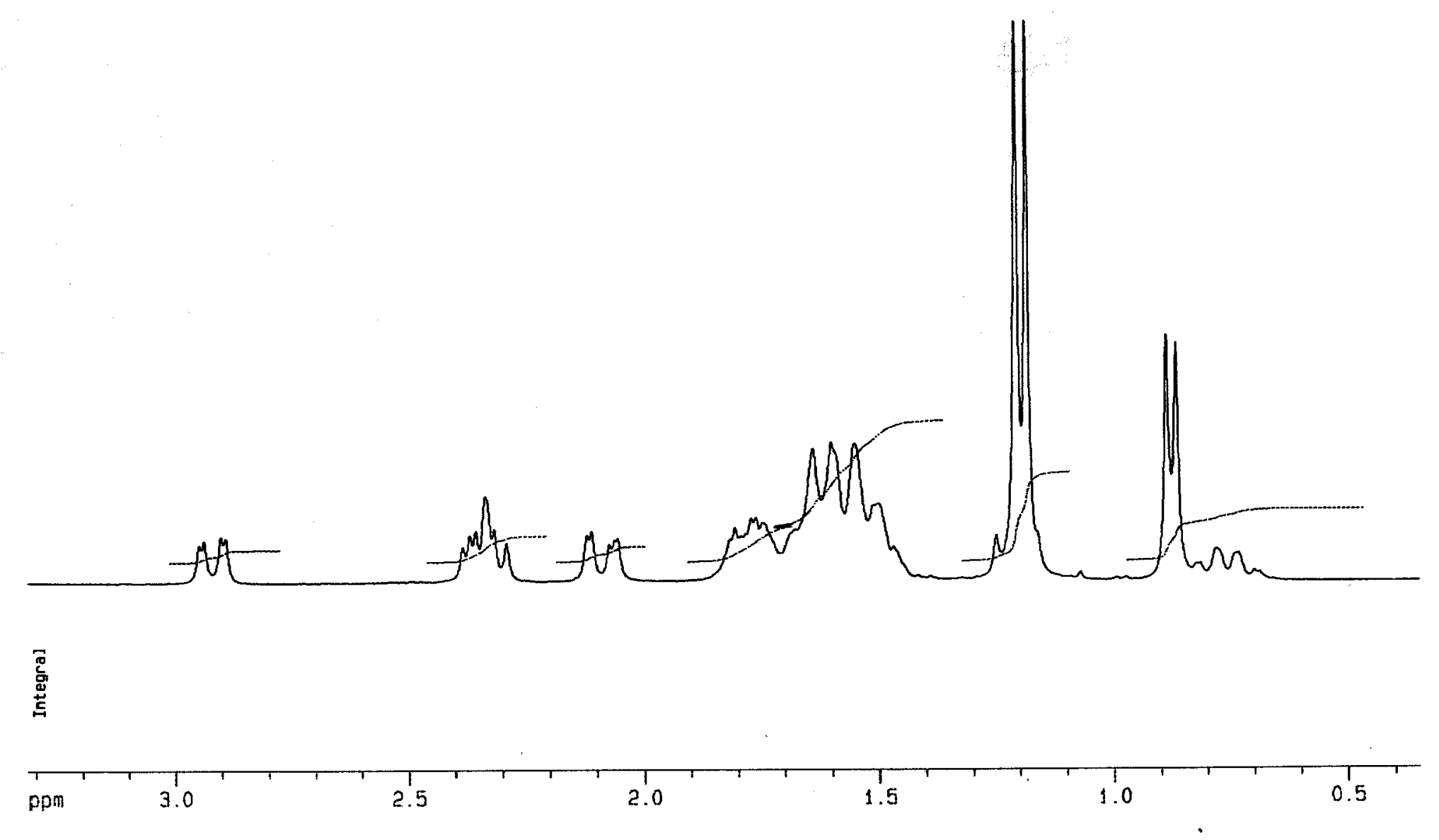

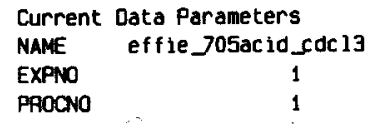

F2 - Acquisition Parameters

Date_ 20040623

Time

INSTRUM

PFOEHO $5 \mathrm{~mm}$ ONP $\mathrm{IH} / \mathrm{1}$

PULPAOG

TO 30720

SOLVENT COC13

$\begin{array}{ll}\text { OS } & 0 \\ \text { SWH } & 5081.301\end{array}$

SNH $\quad 5001.301 \mathrm{~Hz}$

$\begin{array}{lc}\text { FIOFES } & 0.165407 \mathrm{~Hz} \\ A Q & 3.0228980 \mathrm{sec}\end{array}$

$\begin{array}{lc}\text { AQ } & 3.0228980 \mathrm{sec} \\ \text { AG } & 181\end{array}$

$\begin{array}{rr}\text { DW } & 98.400 \text { usec } \\ \text { DE } & 6.00 \text { usec }\end{array}$

$300.0 \mathrm{~K}$

$1.00000000 \mathrm{sec}$

$m=m-m=-m=$ CHANNEL $f 1$

$\begin{array}{ll}\text { NUC1 } & 1 \mathrm{H} \\ \mathrm{P1} & 10.50 \text { use }\end{array}$

PL1 $\quad-3.00 \mathrm{~dB}$

SFO1 $\quad 300.1319477 \mathrm{MHz}$

F2 - Processing parameters

$\begin{array}{lc}\text { SI } & 65536 \\ \text { SF } & 300.1300000 \mathrm{MHz}\end{array}$

NOW EM

SSB $\quad 0.10 \mathrm{~Hz}$

GB $\quad 100$

10 MA plot parameters

$\begin{array}{lc}\text { CX } & 20.00 \mathrm{~cm} \\ \text { CY } & 8.00 \mathrm{~cm} \\ \text { FIP } & 3.317 \mathrm{ppm} \\ \text { F1 } & 995.61 \mathrm{~Hz} \\ \text { F2P } & 0.349 \mathrm{ppm} \\ \text { F2 } & 104.60 \mathrm{~Hz} \\ \text { PFMOM } & 0.14844 \mathrm{pPm} / \mathrm{cm} \\ \text { HZCM } & 44.55048 \mathrm{~Hz} / \mathrm{cm}\end{array}$



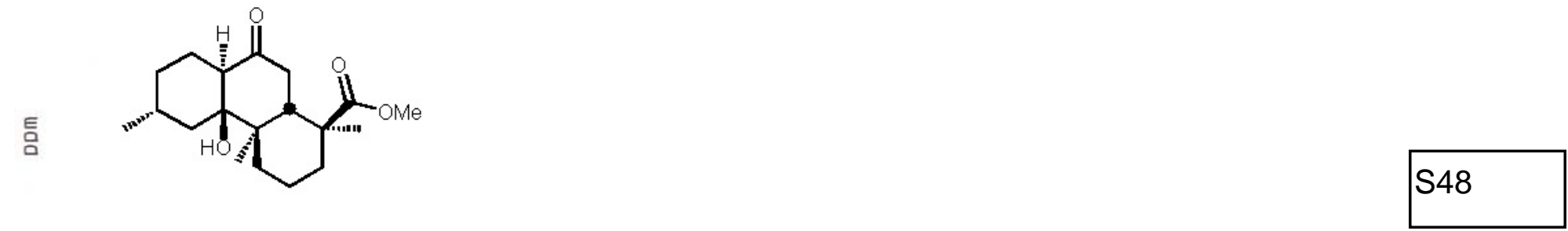

Current Data Parameters

EXPNO

PAOCNO

F2 - Acquisition Parame

Date $\quad 19940304$ Time 20.45

PULPROG 2gdc

SOLVENT CDC13

AQ $\quad 1.0485960$

FIDRES $\quad 0.476837$

DW 16.0

RG 32768

NUCLEUS $\quad 13 \mathrm{C}$

D11 $\quad 0.0300000$

P31 80.0

$\begin{array}{ll}\text { S2 } & 18 \\ \text { HL1 } & 22\end{array}$

$\begin{array}{lr}\text { HL1 } & 22 \\ 01 & 1.0000000\end{array}$

$\begin{array}{lr}\text { P1 } & 2.0 \\ \text { DE } & 20.0\end{array}$

SF01 125.7724464

SWH $\quad 31250,00$

TD

$\begin{array}{ll}\text { NS } & 20480\end{array}$

DS

F1 - Processing parametı SI 32768

$S F$

WDW

LB

LB

125.7591495

10 NMR plot parameters

CX 22.00

F1P 229.979

F1 28921.93

F2P $\quad-18.512$

F2 -2328.07

$\begin{array}{lr}\text { PPMCM } & 11.29504 \\ \text { HZCM } & 1420.45447\end{array}$

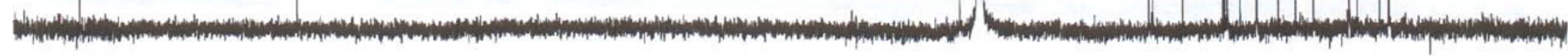




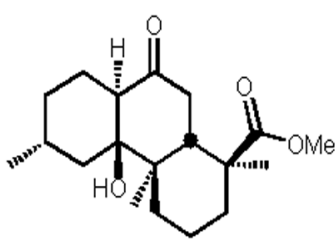

F2 - Acquisition Parameters Date_ 20040108 $\begin{array}{lr}\text { Date } & 20040108 \\ \text { Time } & 16.32\end{array}$ INSTRUM $\quad$ av300 PROBHD $5 \mathrm{~mm}$ QNP $1 \mathrm{H} / 1$ $\begin{array}{ll}\text { PAOBHD } 5 \mathrm{~mm} \text { GNP } & 1 \mathrm{H} / 1 \\ & \mathrm{Zg} 30\end{array}$ $\begin{array}{lr}\text { PULPROG } & 2930 \\ \text { TD } & 30720\end{array}$ SOLVENT COCl3 $\begin{array}{lr}\text { NS } & 32 \\ \text { DS } & 0\end{array}$ SWH $\quad 5081.301 \mathrm{~Hz}$ FIORES $\quad 0.165407 \mathrm{~Hz}$ AQ $\quad 3.0228980 \mathrm{sec}$ $\mathrm{BG} \quad 574.7$ DW 98.400 usec

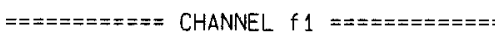
NUC1

P1 10.50 usec PL1 $\quad-3.00 \mathrm{~dB}$ SF01 $\quad 300.1319477 \mathrm{MHz}$

F2 - Processing parameters SI 65536 SF $\quad 300.1300000 \mathrm{MHz}$ WOW EM LB $\quad 00$ GB $\quad 0$

10 NMR plot parameters

CX $\quad 20.00 \mathrm{~cm}$

CY $\quad 10.00 \mathrm{cI}$

$9.000 \mathrm{ppm}$

F1 $2701.17 \mathrm{~Hz}$

$\begin{array}{ll}F 2 P & -1.000 \mathrm{ppm} \\ F 2 & -300.13 \mathrm{~Hz}\end{array}$

PPMCM $\quad 0.50000 \mathrm{ppm} / \mathrm{cm}$

$\mathrm{HZCM} \quad 150.06500 \mathrm{~Hz} / \mathrm{cm}$ 
This spectrum

C-7

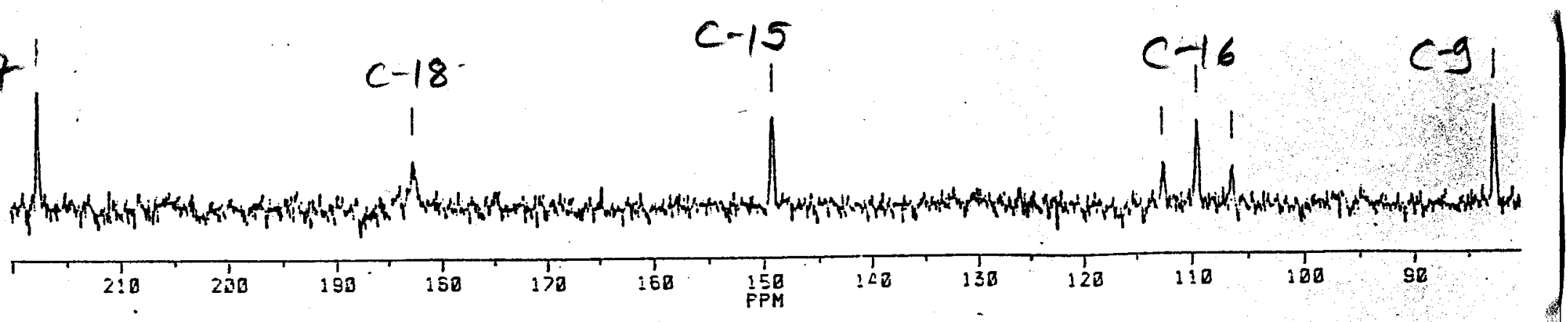
was token in melt,

S50

therefore

it. has some chemical defences from the ${ }^{13} \mathrm{C}$ NME spectrum in $\mathrm{CDCl}_{3}$ in the manuscript

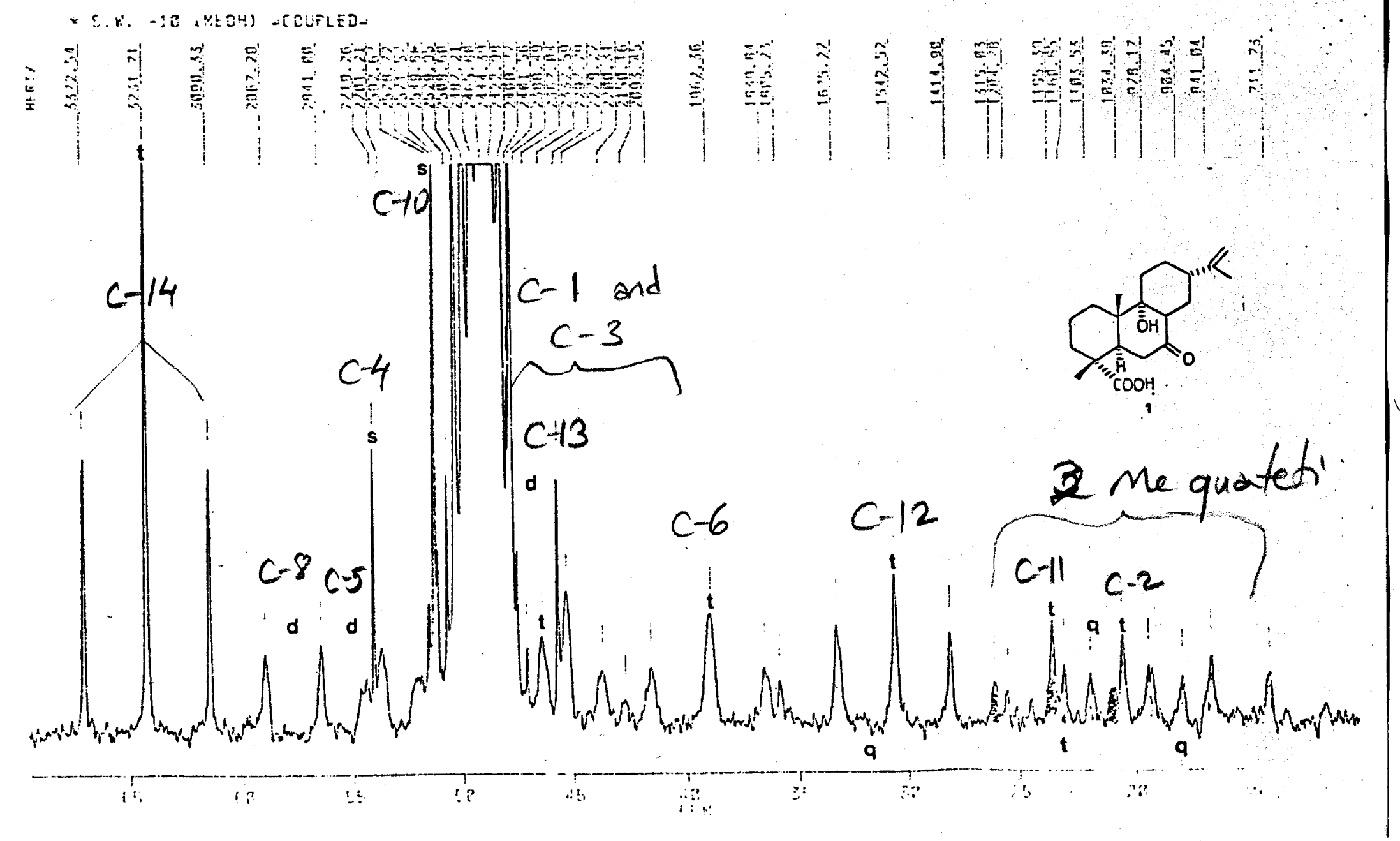




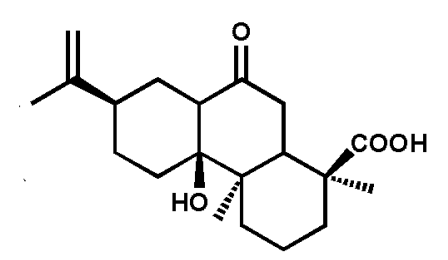

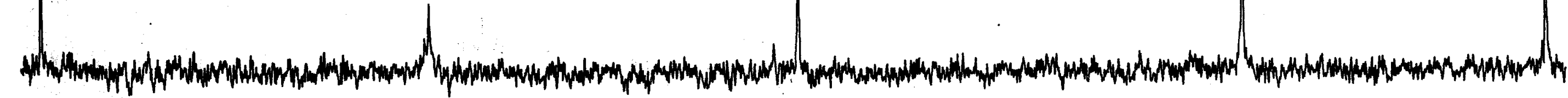

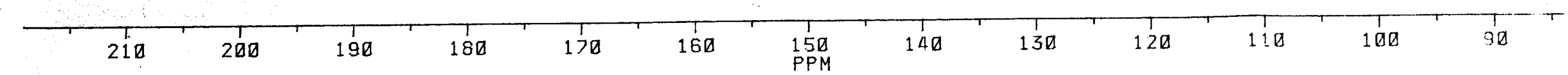


. BB-decupled speetrum in sineolt

* S. W. -10 (MEOH)

变

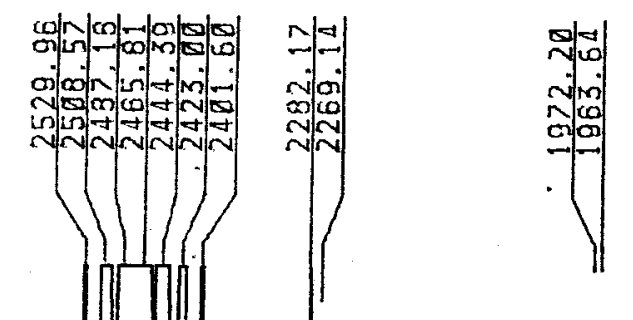

$\begin{array}{lll}0 & 01 \\ 0 & 0 & 0 \\ 0 & 0 & 0 \\ 7 & & \end{array}$

m.

임

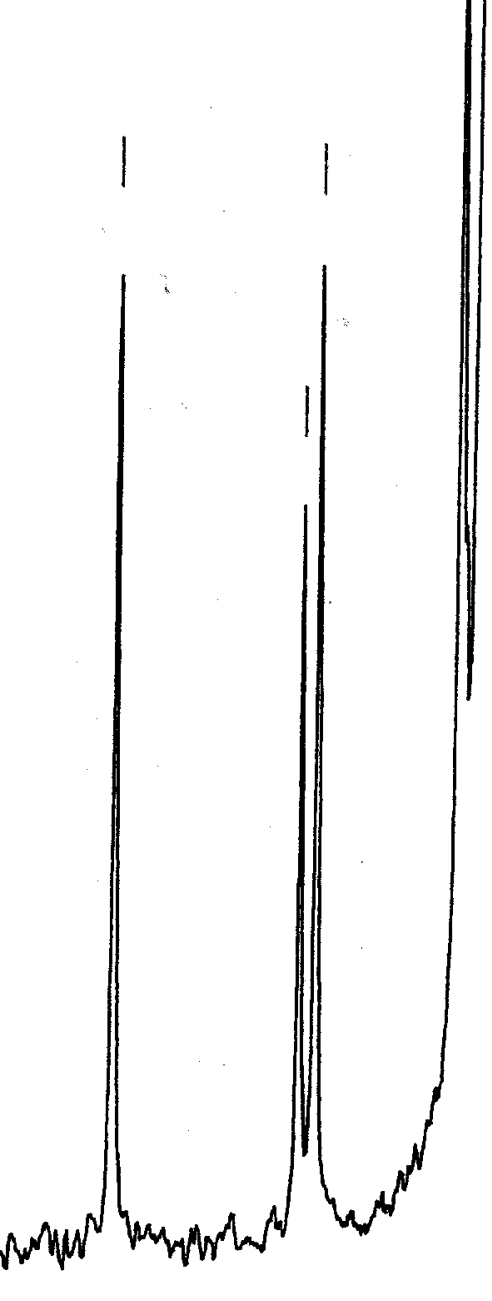


NOE (inCOCe, $\begin{aligned} & 400 \mathrm{MHz} \\ & \text { Brukn }\end{aligned}$<smiles>C=C(C)C1CC[C@@]2(C)C3CC(=O)CC4[C@@](C)(C(=O)O)CCC[C@]43C[C@@]12C</smiles>

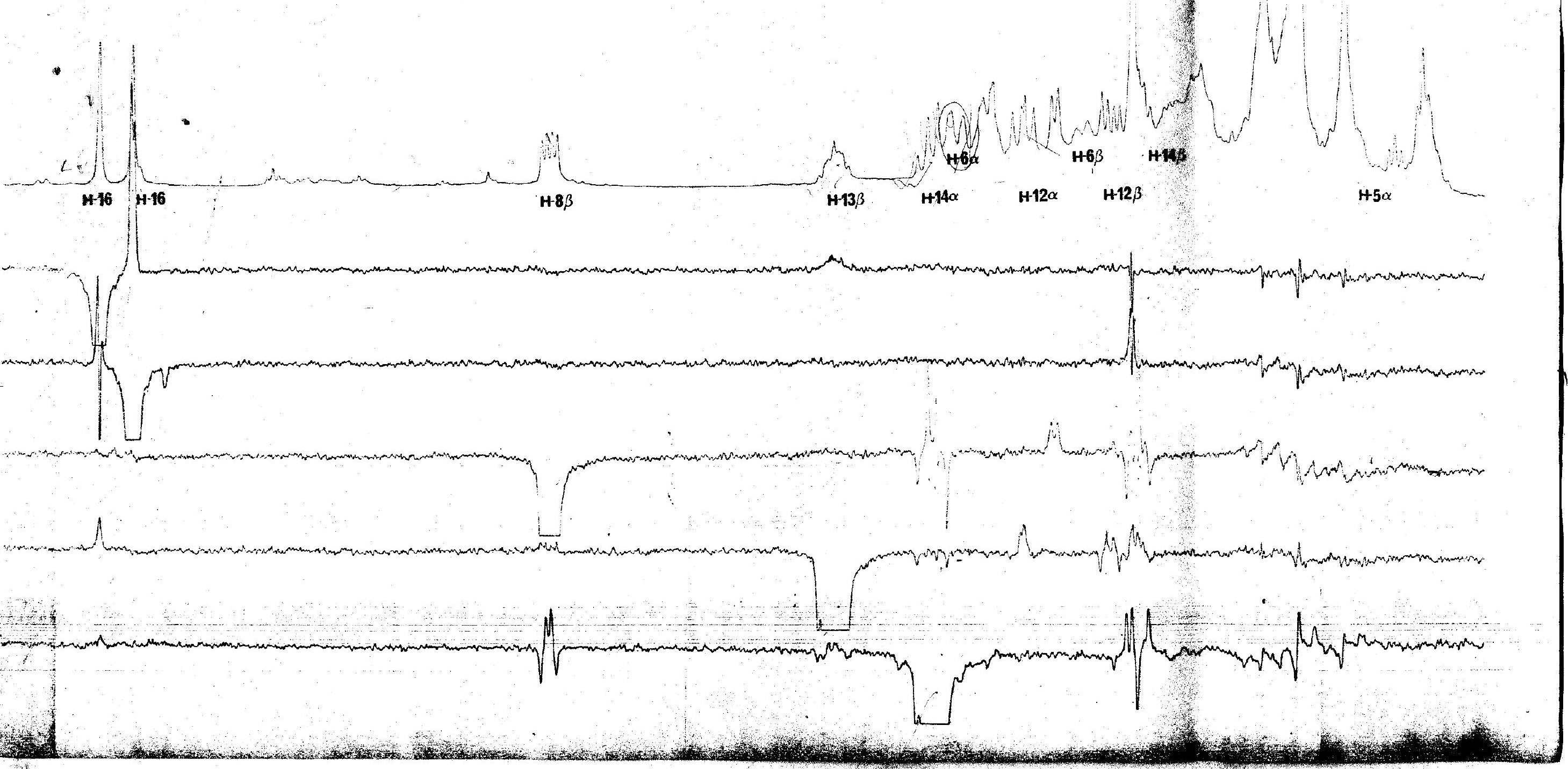




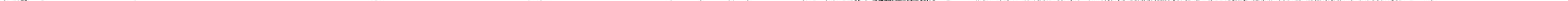


CDCl

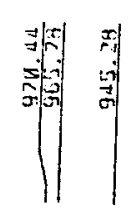

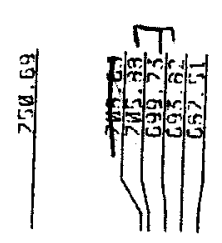

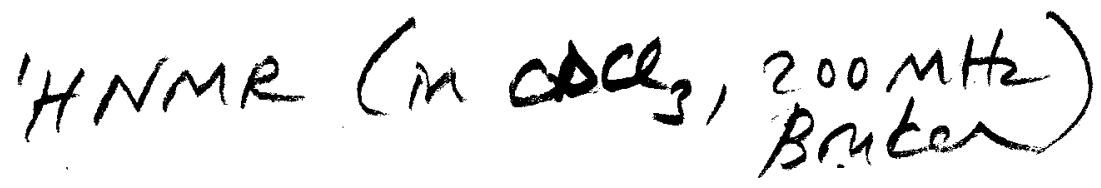

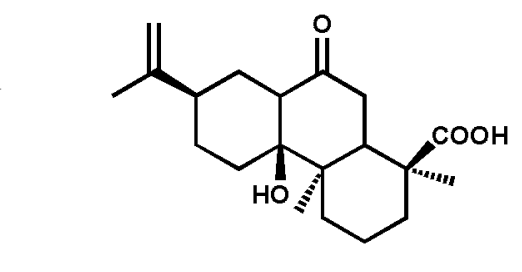

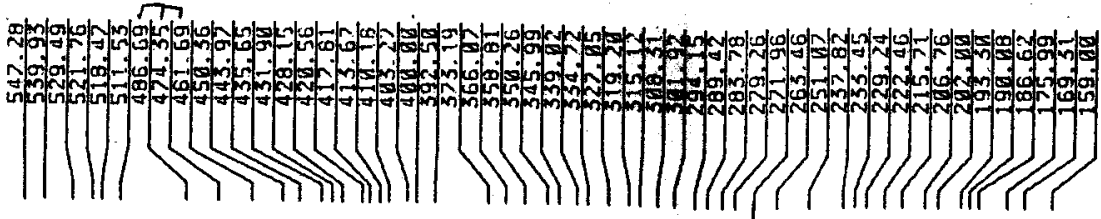

筋赫
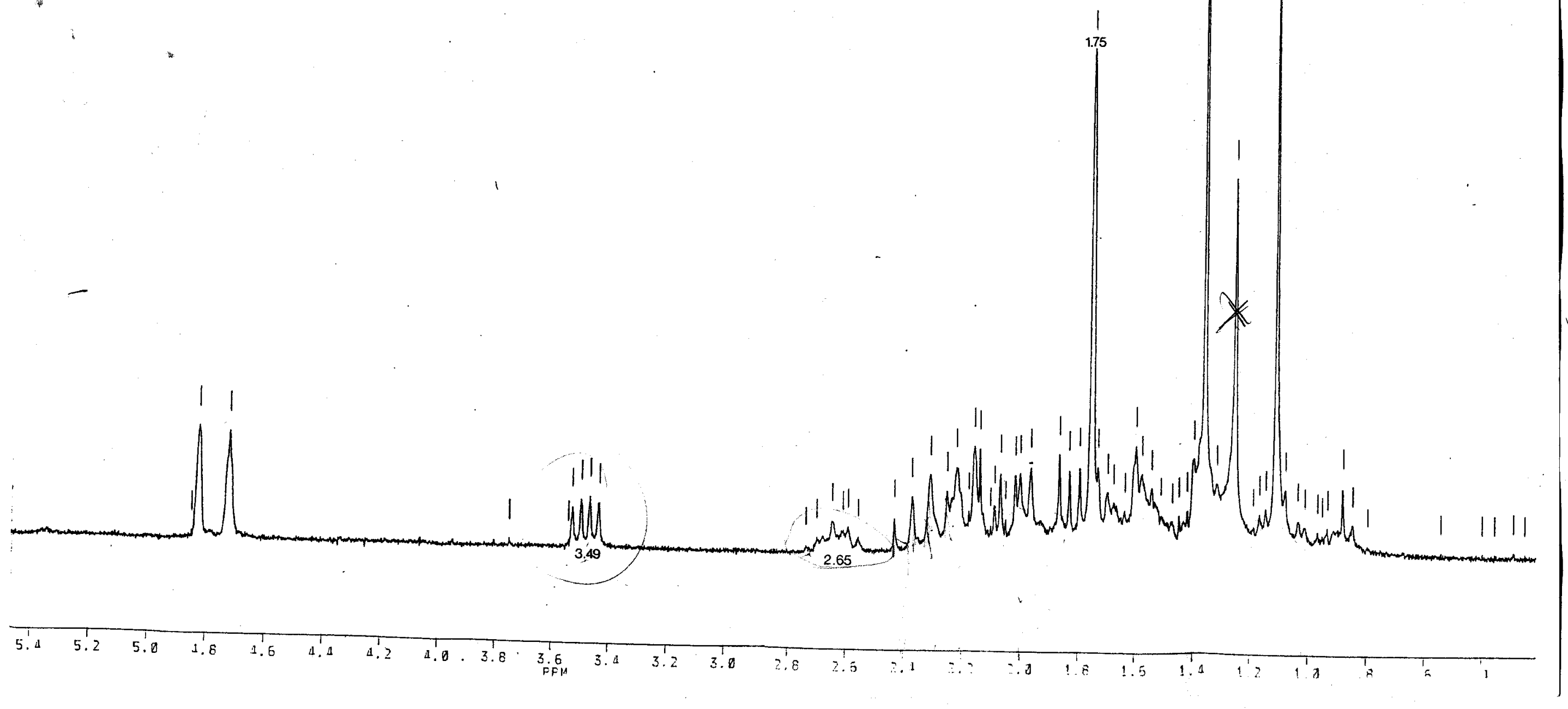